\title{
Discrete $G$-spectra and embeddings of module spectra
}

\author{
Takeshi Torii ${ }^{1}$
}

Received: 17 March 2015 / Accepted: 6 December 2016 / Published online: 4 January 2017

(C) Tbilisi Centre for Mathematical Sciences 2017

\begin{abstract}
In this paper we study the category of discrete $G$-spectra for a profinite group $G$. We consider an embedding of module objects in spectra into a category of module objects in discrete $G$-spectra, and study the relationship between the embedding and the homotopy fixed points functor. We also consider an embedding of module objects in terms of quasi-categories, and show that the two formulations of embeddings are equivalent in some circumstances.
\end{abstract}

Keywords Discrete $G$-spectrum · Homotopy fixed points · Quasi-category

Mathematics Subject Classification Primary 55P42; Secondary 55P91 · 55P43

\section{Introduction}

By the works of Morava [30], Miller et al. [29], Devinatz et al. [12], Hopkins and Smith [19], Hovey and Strickland [23], and many others, the stable homotopy category is intimately related to the theory of formal groups, and the stable homotopy category localized at a prime $p$ has a filtration of full subcategories corresponding to the height of formal groups. The $n$th full subcategory for non-negative integer $n$ is realized as

\section{Communicated by Daniel Davis.}

The author would like to thank the anonymous referee for many helpful comments and suggestions. He also would like to thank Daniel Davis for pointing out the mistake in Lemma 6.1 in the preprint version of the paper. This work was partially supported by JSPS KAKENHI Grant Number 25400092.

\footnotetext{
$凶 \quad$ Takeshi Torii

torii@math.okayama-u.ac.jp

1 Department of Mathematics, Okayama University, Okayama 700-8530, Japan
} 
the $E(n)$-local category, that is, the Bousfield localization with respect to the $n$th Johnson-Wilson theory $E(n)$ at $p$. The $n$th subquotient of the filtration is equivalent to the $K(n)$-local category, that is, the Bousfield localization of the stable homotopy category with respect to the $n$th Morava $K$-theory $K(n)$ at $p$, and we can consider that the fundamental building blocks of the stable homotopy category are the $K(n)$ local categories for various $n$ and $p$. Therefore, studying the $K(n)$-local category has central importance in stable homotopy theory. A basic tool to study the $K(n)$ local category is the $K(n)$-local $E_{n}$-based Adams spectral sequence, where $E_{n}$ is the $n$th Morava $E$-theory at $p$. The $E_{2}$-page of this spectral sequence for a $K(n)$ local $E(n-1)$-acyclic spectrum $X$ is described as the continuous cohomology of the extended Morava stabilizer group $\mathbb{G}_{n}$ with coefficients in the discrete twisted $E_{n *}-\mathbb{G}_{n^{-}}$ module $\left(E_{n}\right)_{*}(X)$. This suggests that the derived category of discrete (or continuous) twisted $E_{n *}-\mathbb{G}_{n}$-modules may be intimately related to the $K(n)$-local category.

An algebraic model of the $E(n)$-local category was constructed by Franke [14] for sufficiently large primes $p$ compared to $n$, after the pioneering work by Bousfield [6]. Although Franke's theory gives an equivalence of categories between the derived category of periodic chain complexes in $E(n)_{*}(E(n))$-comodules and the $E(n)$-local category, it does not give an equivalence of model categories. Therefore, it does not give a model of the homotopy theory of $E(n)$-local spectra, and we would like to have a model of the homotopy theory of $E(n)$-local spectra, and of $K(n)$-local spectra. In this paper we propose a model of the homotopy theory of $K(n)$-local spectra in the model category of $K(n)$-local $F_{n}$-modules in the discrete symmetric $\mathbb{G}_{n}$-spectra, where $F_{n}$ is a discrete model of $E_{n}$ constructed by Davis in [9] and upgraded to a commutative monoid object in the category of discrete symmetric $\mathbb{G}_{n}$-spectra by Behrens and Davis in [4]. For this purpose, we shall formulate embeddings of module spectra in general setting.

Let $G$ be a profinite group. The theory of $G$-Galois extensions of structured ring spectra was introduced by Rognes in [34], and he gave an interpretation of the results in [11] in terms of $G$-Galois extensions. The category of discrete $G$-spectra was introduced by Davis in [9], and $G$-Galois extensions were also studied by Behrens and Davis in [4] in terms of discrete $G$-spectra. The algebraic Galois theory is related to the descent theory, which has been important in algebraic geometry, number theory, category theory and homotopy theory. The framework of homotopical descent theory was developed by Hess [17] and Lurie [28]. The formulation of embeddings we discuss in this paper is some kind of homotopical descent related to $G$-Galois extensions of spectra.

Let $\Sigma \operatorname{Sp}(G)$ be the model category of discrete symmetric $G$-spectra and let $\Sigma \operatorname{Sp}(G)_{k}$ be its left Bousfield localization with respect to a spectrum $k$. Suppose we have a map $A \rightarrow B$ of monoids in discrete symmetric $G$-spectra, where $G$ acts on $A$ trivially. There is a functor

$$
\operatorname{Ex}: \operatorname{Mod}_{A}\left(\Sigma \operatorname{Sp}_{k}\right) \rightarrow \operatorname{Mod}_{B}\left(\Sigma \operatorname{Sp}(G)_{k}\right)
$$

from the category $\operatorname{Mod}_{A}\left(\Sigma \operatorname{Sp}_{k}\right)$ of $A$-modules in $\Sigma \operatorname{Sp}_{k}$ to the category $\operatorname{Mod}_{B}\left(\Sigma \operatorname{Sp}(G)_{k}\right)$ of $B$-modules in $\Sigma \operatorname{Sp}(G)_{k}$ by the extension of scalars. This functor has a right adjoint, which we can regard as a fixed points functor $(-)^{G}$. Furthermore, the pair of functors 
is a $\Sigma$ Sp-Quillen adjunction. We denote by $\mathbb{L E x}$ the total left derived functor of Ex and by $(-)^{h G}$ the total right derived functor of the fixed points functor $(-)^{G}$. The functor $(-)^{h G}$ can be regarded as the homotopy fixed points functor. Let $\mathbf{T}$ be the full subcategory of the homotopy category $\operatorname{Ho}\left(\operatorname{Mod}_{A}\left(\Sigma \operatorname{Sp}_{k}\right)\right)$ consisting of $X$ such that the unit map $X \rightarrow(\mathbb{L E x}(X))^{h G}$ is an equivalence. We show that the restriction of $\mathbb{L}$ Ex to $\mathbf{T}$ is fully faithful as an $\operatorname{Ho}(\Sigma S p)$-enriched functor (Proposition 4.5). Using the $K(n)$-local $\mathbb{G}_{n}$-Galois extension $L_{K(n)} S \rightarrow F_{n}$ formulated in $[4, \S 8]$ and the result in [10], we obtain the following theorem.

Theorem 1.1 (Theorem 4.12) The total left derived functor

$$
\mathbb{L E x}: \operatorname{Ho}\left(\Sigma \operatorname{Sp}_{K(n)}\right) \longrightarrow \operatorname{Ho}\left(\operatorname{Mod}_{F_{n}}\left(\Sigma \operatorname{Sp}\left(\mathbb{G}_{n}\right)_{K(n)}\right)\right)
$$

\section{is fully faithful as an $\mathrm{Ho}(\Sigma \mathrm{Sp})$-enriched functor.}

Next we consider embeddings of modules in quasi-categories. Model categories are models of homotopy theories and contain rich homotopy theoretic structures. But they are sometimes rigid and hard to work with since they contain auxiliary structures such as cofibrations and fibrations. Simplicial categories and topological categories are also models of homotopy theories. But they are also sometimes rigid and hard to work with since they have a strict associative composition law of mapping spaces. Quasi-categories are yet other models of homotopy theories. Quasi-categories were introduced by Boardman-Vogt [3] and developed by Joyal [25,26] and Lurie [27,28]. The definition of quasi-categories is a little strange at first glance but actually quasicategories are flexible, easy to work with, and well-developed. Therefore, to formulate embeddings of modules in terms of quasi-categories is important and will be useful for later applications.

Let $\mathrm{Sp}_{k}$ be the underlying quasi-category of the simplicial model category $\Sigma \mathrm{Sp}_{k}$. Suppose we have a map $A \rightarrow E$ of algebra objects in $\mathrm{Sp}_{k}$. We have a functor from the quasi-category $\operatorname{Mod}_{A}\left(\operatorname{Sp}_{k}\right)$ of $A$-modules to the quasi-category $\operatorname{Mod}_{E}\left(\operatorname{Sp}_{k}\right)$ of $E$ modules by the extension of scalars. This defines a comonad $\Theta$ on the $E$-modules, and we can consider the quasi-category of comodules $\operatorname{Comod}_{(E, \Theta)}\left(\operatorname{Sp}_{k}\right)$ over the comonad $\Theta$. The extension of scalars functor factors through $\operatorname{Comod}_{(E, \Theta)}\left(\operatorname{Sp}_{k}\right)$, and we obtain a functor

$$
\text { Coex }: \operatorname{Mod}_{A}\left(\operatorname{Sp}_{k}\right) \rightarrow \operatorname{Comod}_{(E, \Theta)}\left(\operatorname{Sp}_{k}\right)
$$

This functor has a right adjoint $P$, which is a homotopical analogue of the functor taking primitive elements. Let $\mathbf{T}$ be the full subcategory of $\operatorname{Mod}_{A}\left(\operatorname{Sp}_{k}\right)$ consisting of $X$ such that the unit map $X \rightarrow P \operatorname{Coex}(X)$ is an equivalence. We show that the restriction of the functor Coex to $\mathbf{T}$ is fully faithful (Proposition 5.2). This result is related to the (effective) homotopic descent considered by Hess in [17, Def. 5.1], but the author is not sure if this result can be formulated in terms of simplicial categories. There is a map of quasi-categories from the simplicial nerve of the simplicial category of coalgebras in $\operatorname{Mod}_{E}\left(\Sigma \mathrm{Sp}_{k}\right)$ associated to the adjunction $\operatorname{Mod}_{A}\left(\Sigma \mathrm{Sp}_{k}\right) \rightleftarrows \operatorname{Mod}_{E}\left(\Sigma \operatorname{Sp}_{k}\right)$ to the quasi-category of comodules $\operatorname{Comod}_{(E, \Theta)}\left(\operatorname{Sp}_{k}\right)$, but it seems that this map may not 
be an equivalence in general since a comodule in $\operatorname{Comod}_{(E, \Theta)}\left(\operatorname{Sp}_{k}\right)$ which satisfies the comodule structure up to higher coherent homotopies may not be rectified as a coalgebra in $\operatorname{Mod}_{A}\left(\Sigma \mathrm{Sp}_{k}\right)$ which satisfies the comodule structure on the nose.

Finally, we compare the two formulations of embeddings in terms of model categories and of quasi-categories. For this purpose we describe the underlying quasicategories of modules and algebras in discrete symmetric $G$-spectra for a profinite group $G$ in terms of the quasi-categories of modules and algebras in non-equivariant symmetric spectra.

Let $A \rightarrow B$ be a map of monoids in discrete symmetric $G$-spectra, where $G$ acts on $A$ trivially. Let $U: \Sigma \operatorname{Sp}(G)_{k} \rightarrow \Sigma \operatorname{Sp}_{k}$ be the forgetful functor, which has the right adjoint $V=\operatorname{Map}_{c}(G,-)$ (see Sect. 3.1 for the functor $\left.\operatorname{Map}_{c}(G,-)\right)$. We have a map

$$
\Psi_{U B}: B(U B, A, U B) \longrightarrow U \operatorname{Map}_{c}(G, U B),
$$

where $B(U B, A, U B)$ is the two-sided bar construction of the $A$-module $U B$ (see Sect. 6.4 for the construction of the map $\left.\Psi_{U B}\right)$. Let $\operatorname{Mod}_{B}\left(\operatorname{Sp}(G)_{k}\right)$ be the underlying quasi-category of the simplicial model category $\operatorname{Mod}_{B}\left(\Sigma \operatorname{Sp}(G)_{k}\right)$. We show that $\operatorname{Mod}_{B}\left(\operatorname{Sp}(G)_{k}\right)$ can be written as a quasi-category of comodules.

Theorem 1.2 (Corollary 6.16) Let $G$ be a profinite group that has finite virtual cohomological dimension. We assume that the localization functor $L_{k}$ is given as a composite $L_{M} L_{T}$ of two localization functors $L_{M}$ and $L_{T}$, where $L_{T}$ is a smashing localization and $L_{M}$ is a localization with respect to a finite spectrum $M$. If $\Psi_{U B}$ is a $k$-local equivalence, then there is an equivalence of quasi-categories

$$
\operatorname{Mod}_{B}\left(\operatorname{Sp}(G)_{k}\right) \simeq \operatorname{Comod}_{(U B, \Theta)}\left(\operatorname{Sp}_{k}\right)
$$

As a corollary, we obtain that the functor Ex is equivalent to the functor Coex under the equivalence between $\operatorname{Mod}_{B}\left(\operatorname{Sp}(G)_{k}\right)$ and $\operatorname{Comod}_{(U B, \Theta)}\left(\operatorname{Sp}_{k}\right)$ (Corollary 6.17), where we regard Ex as a functor of the underlying quasi-categories. This shows that the two formulations of embeddings are equivalent. In particular, we show that the two formulations are equivalent if $A \rightarrow B$ is a $k$-local $G$-Galois extension (Theorem 7.3) under the assumptions of Theorem 1.2.

The organization of this paper is as follows: In Sect. 3 we discuss the model structure on the category of discrete symmetric $G$-spectra. We show that the category of discrete symmetric $G$-spectra is a proper, combinatorial, symmetric monoidal $\Sigma$ Sp-model category satisfying the monoid axiom. We also discuss the Bousfield localization with respect to a spectrum with trivial $G$-action. In Sect. 4 we discuss embeddings of modules into the category of discrete symmetric $G$-spectra. We also discuss the relationship between the embeddings and the homotopy fixed points functors. In Sect. 5 we consider embeddings of quasi-categories of modules in spectra. For an adjunction of quasi-categories, we can consider the quasi-category of comodules over the comonad associated to the adjunction. We show that some full subcategory can be embedded into the quasi-category of comodules. In Sect. 6 we study the quasi-category of discrete $G$-spectra. We show that the quasi-category of discrete $G$-spectra can be described as a quasi-category of comodules. Finally we show that the two formulations of embeddings of module categories are equivalent in some circumstances. In Sect. 7 we discuss 
embeddings associated to profinite $G$-Galois extensions. We show that the two formulations are equivalent for profinite $G$-Galois extensions under some conditions.

\section{Notation}

For a model category $\mathbf{M}$, we denote by $\mathrm{Ho}(\mathbf{M})$ the homotopy category of $\mathbf{M}$. For objects $X, Y \in \mathbf{M}$, we denote by $[X, Y]_{\mathbf{M}}$ the set of morphisms in $\mathrm{Ho}(\mathbf{M})$. For a simplicial model category $\mathbf{N}$, we denote by $\operatorname{Map}_{\mathbf{N}}(X, Y)$ the mapping space (simplicial set) for $X, Y \in \mathbf{N}$ (see, for example, [16,18,20] for these concepts). We denote by $\mathbf{N}^{\circ}$ the full simplicial subcategory of $\mathbf{N}$ consisting of objects that are both fibrant and cofibrant as in [27]. The underlying quasi-category of $\mathbf{N}$ is defined to be $N\left(\mathbf{N}^{\circ}\right)$, where $N(-)$ is the simplicial nerve functor (see [27, 1.1.5] for the simplicial nerve functor).

We denote by $\Sigma$ Sp the category of symmetric spectra constructed by HoveyShipley-Smith in [24]. We give $\Sigma$ Sp the stable model structure (see [24, §3] for the definition of the stable model structure on $\Sigma$ Sp). We denote by Sp the underlying quasi-category of $\Sigma \mathrm{Sp}$. For a spectrum $k$, we denote by $\Sigma \operatorname{Sp}_{k}$ the left Bousfield localization of $\Sigma$ Sp with respect to $k$ (see, for example, [18, Ch. 3] for the definition of left Bousfield localization of a model category), and by $\mathrm{Sp}_{k}$ the underlying quasi-category of $\Sigma \mathrm{Sp}_{k}$. We denote by $\mathcal{S}$ the quasi-category of spaces, which is the underlying quasi-category of the category of simplicial sets with the Kan model structure (see $[27,1.2 .16])$. For a quasi-category $\mathcal{C}$, we have a mapping space (simplicial set) $\operatorname{Map}_{\mathcal{C}}(X, Y)$ for $X, Y \in \mathcal{C}$, which is well-defined up to weak homotopy equivalence (see $[27,1.2 .2])$.

\section{Model structure on the category of discrete symmetric $G$-spectra}

Let $G$ be a profinite group. In this section we discuss model structure on the category of discrete symmetric $G$-spectra. We also study the Bousfield localization with respect to a spectrum with trivial $G$-action.

\subsection{Discrete symmetric $G$-spectra}

Let $G$ be a profinite group. In this subsection we recall the model structure on the category of discrete symmetric $G$-spectra and study its properties. We show that the category of discrete symmetric $G$-spectra is a proper combinatorial symmetric monoidal $\Sigma$ Sp-model category satisfying the monoid axiom. We also compare the model category of discrete symmetric $G$-spectra with that of non-equivariant symmetric spectra.

First, we recall the definition of a discrete symmetric $G$-spectrum (see [4, §2.3]). We denote by $\operatorname{Set}(G)$ the category of discrete $G$-sets. A simplicial discrete $G$-set is a simplicial object in $\operatorname{Set}(G)$. The model structure on the category of simplicial discrete $G$-sets was studied in [15]. We denote by $\operatorname{sSet}(G)_{*}$ the category of pointed simplicial discrete $G$-sets. Let $\operatorname{sSet}(G)_{*}^{\Sigma}$ be the category of symmetric sequences in $\operatorname{sSet}(G)_{*}$. We can give a closed symmetric monoidal structure on $\operatorname{sSet}(G)_{*}^{\Sigma}$. Let $S$ 
be the symmetric sequence given by $S=\left(S^{0}, S^{1}, S^{2}, \ldots\right)$, where $S^{n}$ is the $n$-sphere with trivial $G$-action. The symmetric sequence $S$ is a commutative monoid object in $\operatorname{sSet}(G)_{*}^{\Sigma}$. A discrete symmetric $G$-spectrum is a module object in $\operatorname{sSet}(G)_{*}^{\Sigma}$ over the commutative monoid $S$. A map of discrete symmetric $G$-spectra is a map of module objects. We denote by $\Sigma \operatorname{Sp}(G)$ the category of discrete symmetric $G$-spectra.

The category $\Sigma \operatorname{Sp}(G)$ is a complete, cocomplete, closed symmetric monoidal category with $S$ as the unit object. We denote the monoidal structure by $X \wedge Y=X \otimes_{S} Y$. We have an adjoint pair

$$
\operatorname{triv}: \Sigma \mathrm{Sp} \rightleftarrows \Sigma \mathrm{Sp}(G):(-)^{G},
$$

where $(-)^{G}$ is the $G$-fixed points functor and the functor triv(-) associates to a symmetric spectrum $X$ the discrete symmetric $G$-spectrum $X$ with trivial $G$-action. Notice that the functor triv is a strong symmetric monoidal functor and the functor $(-)^{G}$ is a lax symmetric monoidal functor.

Now we recall the model structure on $\Sigma \operatorname{Sp}(G)$ defined in [4, §2.3]. Let $U$ : $\Sigma \operatorname{Sp}(G) \rightarrow \Sigma$ Sp be the forgetful functor. A map $f: X \rightarrow Y$ in $\Sigma \operatorname{Sp}(G)$ is said to be

- a cofibration if $U(f)$ is a cofibration of symmetric spectra,

- a weak equivalence if $U(f)$ is a stable equivalence of symmetric spectra, and

- a fibration if it has the right lifting property with respect to all maps which are both cofibrations and weak equivalences.

With these definitions, $\Sigma \operatorname{Sp}(G)$ is a left proper cellular model category by [4, Thm. 2.3.2]. Recall that a model category is called left proper if every pushout of a weak equivalence along a cofibration is a weak equivalence (see [18, Ch. 13]). A cellular model category is a cofibrantly generated model category for which there are a set $I$ of generating cofibrations and a set $J$ of generating trivial cofibrations such that

- both the domains and the codomains of the elements of $I$ are compact,

- the domains of the elements of $J$ are small relative to $I$, and

- the cofibrations are effective monomorphisms

(see [18] for these concepts, in particular, [18, Ch. 12] for cellular model categories).

We shall show that $\Sigma \operatorname{Sp}(G)$ is a proper combinatorial model category. Recall that a model category is called right proper if every pullback of a weak equivalence along a fibration is a weak equivalence, and proper if it is both left proper and right proper (see [18, Ch. 13]). A model category is said to be combinatorial if it is cofibrantly generated as a model category and is locally presentable as a category (see, for example, [13, $\S 2$ ] or [27, A.2.6] for combinatorial model categories). Since $\Sigma \operatorname{Sp}(G)$ is a cofibrantly generated model category, it suffices to show that $\Sigma \operatorname{Sp}(G)$ is locally presentable in order to show that $\Sigma \operatorname{Sp}(G)$ is combinatorial. Recall that a category is locally $\lambda$ presentable for a regular cardinal $\lambda$ if it is cocomplete and has a set $C$ of $\lambda$-compact objects such that every object is a $\lambda$-filtered colimit of objects in $C$. A category is called locally presentable if it is locally $\lambda$-presentable for some regular cardinal $\lambda$ (see [1, Ch. 1] for locally presentable categories). 
Theorem 3.1 The category $\Sigma \operatorname{Sp}(G)$ is locally presentable. Hence $\Sigma \operatorname{Sp}(G)$ is a combinatorial model category.

Proof The category $\operatorname{Set}(G)$ of discrete $G$-sets is locally $\aleph_{0}$-presentable, where $\aleph_{0}$ is the first infinite cardinal. A discrete $G$-set is $\aleph_{0}$-compact if and only if the underlying set is finite. The full subcategory of finite discrete $G$-sets is essentially small. We denote by $\mathcal{A}$ the opposite category of a skeleton of the full subcategory of finite discrete $G$ sets. For a small category $\mathcal{C}$ and a category $\mathcal{D}$, we denote by $\operatorname{Fun}(\mathcal{C}, \mathcal{D})$ the functor category and by $\operatorname{Fun}^{\mathrm{Lex}}(\mathcal{C}, \mathcal{D})$ the full subcategory of finite-limit preserving functors. By $[1, \mathrm{Thm} .1 .46$ and its proof $]$, we see that the Yoneda map $\operatorname{Set}(G) \rightarrow \operatorname{Fun}(\mathcal{A}$, Set $)$ given by $X \mapsto \operatorname{Hom}_{\operatorname{Set}(G)}(-, X)$ for $X \in \operatorname{Set}(G)$ induces an equivalence of categories between $\operatorname{Set}(G)$ and $\operatorname{Fun}^{\text {Lex }}(\mathcal{A}$, Set $)$.

For a (discrete) symmetric $\left(G\right.$-)spectrum $Y$, we denote by $Y_{k, l}$ the set of $l$ simplexes of the $k$ th simplicial set $Y_{k}$ of $Y$. We can define a functor $F: \Sigma \operatorname{Sp}(G) \rightarrow$ $\operatorname{Fun}^{\operatorname{Lex}}(\mathcal{A}, \Sigma \mathrm{Sp})$ by

$$
F(X)(A)_{k, l}=\operatorname{Hom}_{\operatorname{Set}(G)}\left(A, X_{k, l}\right)
$$

for $A \in \mathcal{A}$ and $X \in \Sigma \operatorname{Sp}(G)$ with obvious structure maps. We can verify that the functor $F$ is an equivalence of categories by using the equivalence $\operatorname{Set}(G) \stackrel{\widetilde{ }}{\rightarrow}$ $\operatorname{Fun}^{\mathrm{Lex}}(\mathcal{A}$, Set).

Recall that the category $\Sigma \mathrm{Sp}$ of symmetric spectra is locally presentable. This follows, for example, from [24, 1.2.10, 3.2.13, and 5.1.6]. See also [38, p. 474]. The theorem follows from the fact that for any small category $\mathcal{C}$ and any locally presentable category $\mathcal{D}$, the category $\operatorname{Fun}^{\mathrm{Lex}}(\mathcal{C}, \mathcal{D})$ is locally presentable by $[1,1.53$ and $1.50(1)]$.

\section{Proposition 3.2 The model category $\Sigma \operatorname{Sp}(G)$ is proper.}

Proof It suffices to show that $\Sigma \mathrm{Sp}(G)$ is right proper. Since $\Sigma \mathrm{Sp}$ is right proper by [24, Thm. 5.5.2], this follows from the fact that the forgetful functor $U$ preserves fiber products and detects weak equivalences.

Next we consider the compatibility of the monoidal structure and the model structure on $\Sigma \operatorname{Sp}(G)$. By the definition of the model structure on $\Sigma \operatorname{Sp}(G)$ and the fact that the composition $U$ o triv is the identity functor, the functor triv preserves cofibrations and weak equivalences, and hence the adjoint pair of functors

$$
\operatorname{triv}: \Sigma \mathrm{Sp} \rightleftarrows \Sigma \mathrm{Sp}(G):(-)^{G}
$$

is a Quillen adjunction.

We shall recall the definition of a symmetric monoidal Quillen adjunction (see [20, $\S 4.2]$ ) and show that the pair (triv, $(-)^{G}$ ) is a symmetric monoidal Quillen adjunction. Let $\mathbf{M}$ and $\mathbf{N}$ be symmetric monoidal model categories. A Quillen adjunction $F: \mathbf{M} \rightleftarrows$ $\mathbf{N}: G$ is said to be a symmetric monoidal Quillen adjunction if the left Quillen functor $F$ is strong symmetric monoidal and the map $F(q): F(Q \mathbb{I}) \rightarrow F(\mathbb{I})$ is a weak equivalence, where $\mathbb{I}$ is the unit object in $\mathbf{M}$ and $q: Q \mathbb{I} \rightarrow \mathbb{I}$ is a cofibrant replacement 
of $\mathbb{I}$. We say that the left adjoint of a symmetric monoidal Quillen adjunction is a symmetric monoidal left Quillen functor. Since the functor triv is strong symmetric monoidal and the sphere spectrum is cofibrant in $\Sigma \mathrm{Sp}$, we see that the pair (triv, $(-)^{G}$ ) is a symmetric monoidal Quillen adjunction.

Let $\mathbf{C}$ be a symmetric monoidal model category. We also recall the definitions of a symmetric monoidal $\mathbf{C}$-model category and a symmetric monoidal $\mathbf{C}$-Quillen adjunction (see [20, §4.2]). A model category $\mathbf{M}$ is said to be a symmetric monoidal C-model category if it is a symmetric monoidal model category equipped with a strong symmetric monoidal left Quillen functor $i: \mathbf{C} \rightarrow \mathbf{M}$. Let $\mathbf{M}_{1}$ and $\mathbf{M}_{2}$ be symmetric monoidal C-model categories equipped with symmetric monoidal left Quillen functors $i_{1}: \mathbf{C} \rightarrow \mathbf{M}_{1}$ and $i_{2}: \mathbf{C} \rightarrow \mathbf{M}_{2}$, respectively. A symmetric monoidal $\mathbf{C}$-Quillen adjunction between $\mathbf{M}_{1}$ and $\mathbf{M}_{2}$ is a symmetric monoidal Quillen adjunction $F$ : $\mathbf{M}_{1} \rightleftarrows \mathbf{M}_{2}: G$ together with a symmetric monoidal natural isomorphism between $F \circ i_{1}$ and $i_{2}$. We say that the left adjoint of a symmetric monoidal $\mathbf{C}$-Quillen adjunction is a symmetric monoidal left $\mathbf{C}$-Quillen functor.

Theorem 3.3 The category $\Sigma \operatorname{Sp}(G)$ is a symmetric monoidal $\Sigma$ Sp-model category. The adjoint pair (triv, $(-)^{G}$ ) is a symmetric monoidal $\Sigma \mathrm{Sp}$-Quillen adjunction.

Proof The model category $\Sigma \operatorname{Sp}(G)$ is a symmetric monoidal model category by [21, Thm. 8.11]. The theorem follows from the fact that triv : $\Sigma \mathrm{Sp} \rightarrow \Sigma \operatorname{Sp}(G)$ is a symmetric monoidal left Quillen functor.

Now we shall verify that the symmetric monoidal model category $\Sigma \operatorname{Sp}(G)$ satisfies the monoid axiom. We recall the monoid axiom on a monoidal model category (see, for example, [35, Def. 3.3]). Let $\mathbf{M}$ be a monoidal model category with tensor product $\wedge$. For a class $\mathcal{I}$ of maps in $\mathbf{M}$, we denote by $\mathcal{I} \wedge \mathbf{M}$ the class of maps of the form

$$
A \wedge Z \longrightarrow B \wedge Z
$$

for $A \rightarrow B$ a map in $\mathcal{I}$ and $Z$ an object of $\mathbf{M}$. For a class $\mathcal{J}$ of maps in $\mathbf{M}$, we denote by $\mathcal{J}$-cof reg the class of maps obtained from the maps of $\mathcal{J}$ by cobase change and transfinite composition (see, for example, [18, 10.2] or [20, 2.1.1] for the definition of a transfinite composition). We say that a monoidal model category $\mathbf{M}$ satisfies the monoid axiom if every map in

$$
(\{\text { trivial cofibrations }\} \wedge \mathbf{M})-\text { cof }_{\text {reg }}
$$

is a weak equivalence, where \{trivial cofibrations\} is the class of trivial cofibrations.

Proposition 3.4 The symmetric monoidal model category $\Sigma \operatorname{Sp}(G)$ satisfies the monoid axiom.

Proof We have to show that every map in

$$
(\{\text { trivial cofibrations }\} \wedge \Sigma \mathrm{Sp}(G))-\text { cof }_{\text {reg }}
$$


is a weak equivalence. By the definition of the model structure on $\Sigma \operatorname{Sp}(G)$, it suffices to show that the underlying map is a stable equivalence of symmetric spectra. Since the underlying map of a trivial cofibration is a trivial cofibration of symmetric spectra, the proposition follows from the fact that the category of symmetric spectra with stable model structure satisfies the monoid axiom by [24, Thm. 5.4.1].

Now we shall compare the category of discrete symmetric $G$-spectra with that of non-equivariant symmetric spectra. For this purpose, we introduce a (non-discrete) symmetric $G$-spectra. We denote by $G^{\delta}$ the group $G$ with discrete topology. Let $\Sigma \operatorname{Sp}\left(G^{\delta}\right)$ be the category of symmetric spectra with (continuous) $G^{\delta}$-action. The continuous homomorphism $G^{\delta} \rightarrow G$ induces a functor $(-)^{\delta}: \Sigma \operatorname{Sp}(G) \rightarrow \Sigma \operatorname{Sp}\left(G^{\delta}\right)$. For $X \in \Sigma \operatorname{Sp}\left(G^{\delta}\right)$, we denote by $d X$ the largest discrete $G$-subspectrum of $X$, that is,

$$
d X=\operatorname{colim}_{H} X^{H},
$$

where $H$ ranges over all open subgroups $H$ of $G$. We can regard $d$ as a functor $d: \Sigma \operatorname{Sp}\left(G^{\delta}\right) \rightarrow \Sigma \operatorname{Sp}(G)$. Notice that we have an adjoint pair

$$
(-)^{\delta}: \Sigma \operatorname{Sp}(G) \rightleftarrows \Sigma \operatorname{Sp}\left(G^{\delta}\right): d .
$$

Let $U: \Sigma \operatorname{Sp}(G) \rightarrow \Sigma \mathrm{Sp}$ be the forgetful functor. The functor $U$ is strong symmetric monoidal and has a right adjoint

$$
V: \Sigma \operatorname{Sp} \rightarrow \Sigma \operatorname{Sp}(G)
$$

We shall explicitly describe the right adjoint $V$. For a symmetric spectrum $Y$ (with $G$-action), we denote by $Y_{k, l}$ the set of $l$-simplexes of the $k$ th simplicial set $Y_{k}$ of $Y$. For a set $A$, we denote by $\operatorname{Map}(G, A)$ the $G$-set of all maps from $G$ to $A$ with $G$-action given by $(g \cdot \theta)\left(g^{\prime}\right)=\theta\left(g^{\prime} g\right)$ for $g, g^{\prime} \in G$ and $\theta \in \operatorname{Map}(G, A)$. For a symmetric spectrum $X$, we can define an object $\operatorname{Map}(G, X)$ of $\Sigma \operatorname{Sp}\left(G^{\delta}\right)$ by $\operatorname{Map}(G, X)_{k, l}=$ $\operatorname{Map}\left(G, X_{k, l}\right)$ with obvious structure maps. We define the discrete symmetric $G$ spectrum $\operatorname{Map}_{c}(G, X)$ by

$$
\operatorname{Map}_{c}(G, X)=d(\operatorname{Map}(G, X)) .
$$

The right adjoint $V$ of the forgetful functor $U: \Sigma \operatorname{Sp}(G) \rightarrow \Sigma S p$ is given by

$$
V(X)=\operatorname{Map}_{c}(G, X)
$$

We can easily verify the following proposition.

Proposition 3.5 The adjoint pair of functors

$$
U: \Sigma \operatorname{Sp}(G) \rightleftarrows \Sigma \mathrm{Sp}: V
$$

is a symmetric monoidal $\Sigma \mathrm{Sp}-$ Quillen adjunction. 
Proof By the definition of the model structure on $\Sigma \operatorname{Sp}(G)$, the forgetful functor $U$ preserves cofibrations and weak equivalences, and hence the adjoint pair $(U, V)$ of functors is Quillen adjunction. Since $U$ is a strong symmetric monoidal and the sphere spectrum is cofibrant in $\Sigma \operatorname{Sp}(G)$, we see that the pair $(U, V)$ is a symmetric monoidal Quillen adjunction. Since the composition $U \circ$ triv is the identity functor on $\Sigma$ Sp, we see that the forgetful functor $U$ is a symmetric monoidal left $\Sigma$ Sp-Quillen functor. This completes the proof.

\subsection{Bousfield localization of $\Sigma \operatorname{Sp}(G)$}

Let $k$ be a symmetric spectrum. In this subsection we study the left Bousfield localization $\Sigma \operatorname{Sp}(G)_{k}$ of $\Sigma \operatorname{Sp}(G)$ with respect to $k$. We show that $\Sigma \operatorname{Sp}(G)_{k}$ is a left proper combinatorial symmetric monoidal $\Sigma$ Sp-model category satisfying the monoid axiom. We also compare the model category $\Sigma \operatorname{Sp}(G)_{k}$ with the left Bousfield localization $\Sigma \mathrm{Sp}_{k}$ of non-equivariant symmetric spectra with respect to $k$.

First, we recall that there exists the left Bousfield localization on $\Sigma \operatorname{Sp}(G)$ with respect to $k$. This follows, for example, from the fact that $\Sigma \operatorname{Sp}(G)$ is a left proper cellular model category. We say that a morphism $f$ in $\Sigma \operatorname{Sp}(G)$ is a $k$-local equivalence if $U(f)$ is a $k$-local equivalence in $\Sigma$ Sp. Let $W_{k}$ be the class of $k$-local equivalences in $\Sigma \operatorname{Sp}(G)$. As in [4, p. 5015], there exists the left Bousfield localization $\Sigma \operatorname{Sp}(G)_{k}$ with respect to $W_{k}$, and $\Sigma \operatorname{Sp}(G)_{k}$ is left proper and cellular.

Proposition 3.6 The left Bousfield localization $\Sigma \operatorname{Sp}(G)_{k}$ is a left proper combinatorial simplicial model category.

Proof By Theorems 3.1 and 3.3, and [4, Thm. 2.3.2], we see that $\Sigma \operatorname{Sp}(G)$ is a left proper combinatorial simplicial model category. As in [4, p. 5015], the class of $k$-local equivalences are that of $f$-local equivalences for some map $f$. Hence the left Bousfield localization $\Sigma \operatorname{Sp}(G)_{k}$ is a left proper combinatorial simplicial model category by [27, Prop. A.3.7.3].

In the following of this paper we assume that $k$ is cofibrant for simplicity.

Theorem 3.7 The model category $\Sigma \mathrm{Sp}(G)_{k}$ is a symmetric monoidal $\Sigma \mathrm{Sp}$-model category.

Proof First, we show that $\Sigma \operatorname{Sp}(G)_{k}$ is a symmetric monoidal model category. Let $A \rightarrow B$ be a cofibration and let $X \rightarrow Y$ be a trivial cofibration in $\Sigma \operatorname{Sp}(G)_{k}$. Since $k$ is cofibrant, we see that $X \wedge k \rightarrow Y \wedge k$ is a trivial cofibration in $\Sigma \operatorname{Sp}(G)$. By Theorem 3.3, $\Sigma \operatorname{Sp}(G)$ is a monoidal model category, and hence the map

$$
(A \wedge Y \wedge k) \coprod_{(A \wedge X \wedge k)}(B \wedge X \wedge k) \rightarrow B \wedge Y \wedge k
$$

is a trivial cofibration. We have an isomorphism

$$
\left((A \wedge Y) \coprod_{(A \wedge X)}(B \wedge X)\right) \wedge k \cong(A \wedge Y \wedge k) \coprod_{(A \wedge X \wedge k)}(B \wedge X \wedge k)
$$


and hence the map

$$
(A \wedge Y) \coprod_{(A \wedge X)}(B \wedge X) \rightarrow B \wedge Y
$$

is a trivial cofibration in $\Sigma \operatorname{Sp}(G)_{k}$. Therefore, we see that $\Sigma \operatorname{Sp}(G)_{k}$ is a symmetric monoidal model category.

Next, we show that $\Sigma \operatorname{Sp}(G)_{k}$ is a symmetric monoidal $\Sigma$ Sp-model category. Recall that the functor triv : $\Sigma \mathrm{Sp} \rightarrow \Sigma \mathrm{Sp}(G)$ is a symmetric monoidal left Quillen functor. Furthermore, the identity functor id $: \Sigma \operatorname{Sp}(G) \rightarrow \Sigma \operatorname{Sp}(G)_{k}$ is a symmetric monoidal left Quillen functor. Hence the composition id o triv : $\Sigma \operatorname{Sp} \rightarrow \Sigma \operatorname{Sp}(G)_{k}$ is also a symmetric monoidal left Quillen functor. This shows that $\Sigma \operatorname{Sp}(G)_{k}$ is a symmetric monoidal $\Sigma$ Sp-model category.

Proposition 3.8 The symmetric monoidal model category $\Sigma \operatorname{Sp}(G)_{k}$ satisfies the monoid axiom.

Proof Recall that $W_{k}$ is the class of $k$-local equivalences. We let $C$ be the class of cofibrations in $\Sigma \operatorname{Sp}(G)_{k}$. Since the functor $(-) \wedge k$ preserves all colimits, we see that

$$
\left(\left(\left(C \cap W_{k}\right) \wedge \Sigma \operatorname{Sp}(G)\right)-\text { cof }_{\text {reg }}\right) \wedge k \subset((C \cap W) \wedge \Sigma \operatorname{Sp}(G))-\text { cof }_{\text {reg }}
$$

where $W$ is the class of weak equivalences in $\Sigma \operatorname{Sp}(G)$. By Proposition 3.4, $\Sigma \operatorname{Sp}(G)$ satisfies the monoid axiom. Hence $((C \cap W) \wedge \Sigma \operatorname{Sp}(G))$-cof reg $_{C W}$. This shows that

$$
\left(\left(C \cap W_{k}\right) \wedge \Sigma \operatorname{Sp}(G)\right)-\operatorname{cof}_{\text {reg }} \subset W_{k} .
$$

This completes the proof.

Now we compare the model category $\Sigma \operatorname{Sp}(G)_{k}$ with the left Bousfield localization $\Sigma \mathrm{Sp}_{k}$ of non-equivariant symmetric spectra with respect to $k$.

Proposition 3.9 The adjoint pair of functors

$$
\operatorname{triv}: \Sigma \operatorname{Sp}_{k} \rightleftarrows \Sigma \operatorname{Sp}(G)_{k}:(-)^{G}
$$

is a symmetric monoidal $\Sigma \mathrm{Sp}-Q u i l l e n$ adjunction.

Proof It suffices to show that triv preserves $k$-local equivalences. Let $f$ be a $k$-local equivalence in $\Sigma$ Sp. Since $U(\operatorname{triv}(f))=f$, we see $\operatorname{triv}(f) \in W_{k}$. Hence triv preserves $k$-local equivalences.

Proposition 3.10 The adjoint pair of functors

$$
U: \Sigma \operatorname{Sp}(G)_{k} \rightleftarrows \Sigma \operatorname{Sp}_{k}: V
$$

is a symmetric monoidal $\Sigma \mathrm{Sp}-$ Quillen adjunction. 
Proof By definition, $U$ preserves weak equivalences and cofibrations, and hence the pair $(U, V)$ is a Quillen adjunction. Recall that we have the symmetric monoidal left Quillen functor triv : $\Sigma \operatorname{Sp} \rightarrow \Sigma \operatorname{Sp}(G)_{k}$. Since the composition $U$ o triv is the identity functor on $\Sigma$ Sp, the functor $U$ is a symmetric monoidal left $\Sigma$ Sp-Quillen functor.

\subsection{Filtered colimits in $\Sigma \operatorname{Sp}(G)_{k}$}

In this subsection we shall show that any filtered colimit preserves weak equivalences in $\Sigma \operatorname{Sp}(G)_{k}$. This follows from [32, Prop. 4.1] (see also [13, Prop. 7.3] and [8, Lem. 1.6]).

Proposition 3.11 Let $\Lambda$ be a filtered category, and let $F: X \rightarrow Y$ be a natural transformation of functors from $\Lambda$ to $\Sigma \operatorname{Sp}(G)_{k}$. If $F(\lambda): X(\lambda) \rightarrow Y(\lambda)$ is a $k$-local equivalence for all $\lambda \in \Lambda$, then the induced map on colimits

$$
\underset{\lambda \in \Lambda}{\operatorname{colimit}} X \longrightarrow \underset{\lambda \in \Lambda}{\operatorname{colimit}} Y
$$

is also a $k$-local equivalence.

Proof We shall apply [32, Prop. 4.1] for $\Sigma \operatorname{Sp}(G)_{k}$. By Proposition 3.6, $\Sigma \operatorname{Sp}(G)_{k}$ is a combinatorial model category. Hence we have to show that there exists a generating set of cofibrations between compact objects for $\Sigma \operatorname{Sp}(G)_{k}$. Let $I$ be the set of maps of the form $\left(\partial \Delta^{m} \times G / N\right)_{+} \rightarrow\left(\Delta^{m} \times G / N\right)_{+}$in $\operatorname{sSet}(G)_{*}$, where $m \geq 0$ and $N$ is an open subgroup of $G$. Let $\operatorname{Ev}_{n}: \Sigma \operatorname{Sp}(G) \rightarrow \operatorname{sSet}(G)_{*}$ be the evaluation functor which assigns to a discrete symmetric $G$-spectrum $X$ the $n$th pointed simplicial discrete $G$-set $X_{n}$, and let $F_{n}: \operatorname{sSet}(G)_{*} \rightarrow \Sigma \operatorname{Sp}(G)$ be its left adjoint. We can take $I^{\Sigma}=\bigcup_{n \geq 0} F_{n}(I)$ as a set of generating cofibrations of $\Sigma \operatorname{Sp}(G)_{k}$. We can verify that $F_{n}\left(\left(\partial \Delta^{m} \times G / N\right)_{+}\right)$and $F_{n}\left(\left(\Delta^{m} \times G / N\right)_{+}\right)$are compact objects in $\Sigma \operatorname{Sp}(G)_{k}$ for all $n \geq 0, m \geq 0$ and $N$ since $\partial \Delta^{m}$ and $\Delta^{m}$ are compact objects in the category of simplicial sets. This completes the proof.

Corollary 3.12 For any functor $X$ from a filtered category $\Lambda$ to $\Sigma \operatorname{Sp}(G)_{k}$, the canonical map

$$
\underset{\lambda \in \Lambda}{\operatorname{hocolim}} X \longrightarrow \underset{\lambda \in \Lambda}{\operatorname{colim}} X
$$

in $\operatorname{Ho}\left(\Sigma \operatorname{Sp}(G)_{k}\right)$ is an isomorphism.

Proof Let $X^{\prime} \rightarrow X$ be a cofibrant replacement in the functor category $\operatorname{Fun}\left(\Lambda, \Sigma \operatorname{Sp}(G)_{k}\right)$ with the projective model structure. This model structure exists by [27, Prop. A.2.8.2] since $\Sigma \operatorname{Sp}(G)_{k}$ is a combinatorial model category by Proposition 3.6. The homotopy colimit hocolim $X$ is represented by colim $X^{\prime}$. The map colim $X^{\prime} \rightarrow$ colim $X$ induced on the colimits is a $k$-local equivalence by Proposition 3.11 .

\section{Embeddings of modules into $\Sigma \operatorname{Sp}(G)_{k}$}

In this section we discuss embeddings of certain full subcategories of module objects in $\Sigma \operatorname{Sp}_{k}$ into categories of module objects in $\Sigma \operatorname{Sp}(G)_{k}$. Let $A$ be a monoid object in 
$\Sigma \mathrm{Sp}_{k}$. We regard $A$ as a monoid object in $\Sigma \operatorname{Sp}(G)_{k}$ with trivial $G$-action. For a map $\varphi: A \rightarrow B$ of monoid objects in $\Sigma \operatorname{Sp}(G)_{k}$, we show that a certain full subcategory of $\operatorname{Ho}\left(\operatorname{Mod}_{A}\left(\Sigma \operatorname{Sp}_{k}\right)\right)$ can be embedded into $\operatorname{Ho}\left(\operatorname{Mod}_{B}\left(\Sigma \operatorname{Sp}(G)_{k}\right)\right)$ as an $\operatorname{Ho}(\Sigma S p)$ enriched category. We also discuss the relationship between the embeddings and the homotopy fixed points functors.

\subsection{Model structure on module categories}

In this subsection we define the model structure on the category of module objects in a combinatorial symmetric monoidal $\Sigma$ Sp-model category satisfying the monoid axiom. We also study the adjunction of module categories induced by a map of monoid objects.

Let $\mathbf{C}$ be a closed symmetric monoidal category. First, we recall the definitions of a closed $\mathbf{C}$-module, a closed symmetric $\mathbf{C}$-algebra, and an adjunction between them. A category $\mathbf{N}$ is said to be a closed $\mathbf{C}$-module if it is enriched, tensored, and cotensored over $\mathbf{C}$. For closed $\mathbf{C}$-modules $\mathbf{N}_{1}$ and $\mathbf{N}_{2}$, an adjoint pair of functors $F: \mathbf{N}_{1} \rightleftarrows \mathbf{N}_{2}: G$ is said to be an adjunction of closed $\mathbf{C}$-modules if the left adjoint $F$ respects the tensor structures on $\mathbf{N}_{1}$ and $\mathbf{N}_{2}$ over $\mathbf{C}$. A category $\mathbf{A}$ is said to be a closed symmetric $\mathbf{C}$-algebra if $\mathbf{A}$ is a closed symmetric monoidal category equipped with a strong symmetric monoidal left adjoint functor $i: \mathbf{C} \rightarrow \mathbf{A}$. Let $\mathbf{A}_{1}$ and $\mathbf{A}_{2}$ be closed symmetric $\mathbf{C}$-algebras equipped with strong symmetric monoidal left adjoints $i_{1}: \mathbf{C} \rightarrow \mathbf{A}_{1}$ and $i_{2}: \mathbf{C} \rightarrow \mathbf{A}_{2}$, respectively. An adjunction of closed symmetric $\mathbf{C}$-algebras between $\mathbf{A}_{1}$ and $\mathbf{A}_{2}$ is an adjoint pair of functors $F: \mathbf{A}_{1} \rightleftarrows \mathbf{A}_{2}: G$, where $F$ is a strong symmetric monoidal functor, together with a symmetric monoidal natural isomorphism between $F \circ i_{1}$ and $i_{2}$ (see [20, $\left.\$ 4.1\right]$ for these concepts).

In this subsection we let $\mathbf{M}$ be a combinatorial symmetric monoidal $\Sigma$ Sp-model category with tensor product $\otimes$ satisfying the monoid axiom. For a monoid object $R$ in $\mathbf{M}$, we denote by $\operatorname{Mod}_{R}(\mathbf{M})$ the category of left $R$-module objects in $\mathbf{M}$ and maps between them. The category $\operatorname{Mod}_{R}(\mathbf{M})$ is a closed $\Sigma$ Sp-module for a monoid object $R$ in M. If $R$ is a commutative monoid object, then $\operatorname{Mod}_{R}(\mathbf{M})$ is a closed symmetric monoidal category with tensor product $\otimes_{R}$ and unit object $R$, and furthermore, we can regard $\operatorname{Mod}_{R}(\mathbf{M})$ as a closed symmetric $\Sigma$ Sp-algebra by the functor $R \otimes(-)$ : $\Sigma \mathrm{Sp} \rightarrow \operatorname{Mod}_{R}(\mathbf{M})$ that is a strong symmetric monoidal left adjoint functor.

Let $R$ be a monoid object in M. A map $f: M \rightarrow N$ in $\operatorname{Mod}_{R}(\mathbf{M})$ is said to be

- a weak equivalence if it is a weak equivalence in $\mathbf{M}$,

- a fibration if it is a fibration in $\mathbf{M}$, and

- a cofibration if it has the left lifting property with respect to all maps which are both fibrations and weak equivalences.

With these definitions, $\operatorname{Mod}_{R}(\mathbf{M})$ is a model category by [35, Thm. 4.1]. Note that the unit object $R$ is cofibrant in $\operatorname{Mod}_{R}(\mathbf{M})$.

Let $\mathbf{C}$ be a symmetric monoidal model category. Now we recall the definition of a $\mathbf{C}$-model category and a $\mathbf{C}$-Quillen adjunction between $\mathbf{C}$-model categories (see [20, $\S 4.2]$ ). A model category $\mathbf{N}$ is said to be a $\mathbf{C}$-model category if it is a closed $\mathbf{C}$-module and the action map $\otimes: \mathbf{N} \times \mathbf{C} \rightarrow \mathbf{N}$ is a Quillen bifunctor, that is, for any cofibration 
$f: U \rightarrow V$ in $\mathbf{N}$ and any cofibration $g: W \rightarrow X$ in $\mathbf{C}$, the induced map

$$
(V \otimes W) \coprod_{U \otimes W}(U \otimes X) \longrightarrow V \otimes X
$$

is a cofibration in $\mathbf{N}$ that is trivial if either $f$ or $g$ is (see [20, Def. 4.2.1] for the definition of a Quillen bifunctor). A C-Quillen adjunction between $\mathbf{C}$-model categories is a Quillen adjunction which is also an adjunction of closed $\mathbf{C}$-modules.

We can verify that $\operatorname{Mod}_{R}(\mathbf{M})$ is a $\Sigma$ Sp-model category for any monoid object $R$ in M. If $R$ is a commutative monoid object in $\mathbf{M}$, then $\operatorname{Mod}_{R}(\mathbf{M})$ is a symmetric monoidal model category satisfying the monoid axiom by [35, Thm. 4.1], and furthermore, since the strong symmetric monoidal functor $R \otimes(-): \Sigma \mathrm{Sp} \rightarrow \operatorname{Mod}_{R}(\mathbf{M})$ is a left Quillen functor, $\operatorname{Mod}_{R}(\mathbf{M})$ is a symmetric monoidal $\Sigma$ Sp-model category (see the paragraph just before Theorem 3.3 for the definition of a symmetric monoidal $\mathbf{C}$-model category for a symmetric monoidal model category $\mathbf{C}$ ).

Now we consider an adjunction between categories of module objects induced by a map of monoid objects in M. Let $\varphi: A \rightarrow B$ be a map of monoid objects in $\mathbf{M}$. We have an adjoint pair of functors

$$
B \otimes_{A}(-): \operatorname{Mod}_{A}(\mathbf{M}) \rightleftarrows \operatorname{Mod}_{B}(\mathbf{M}): \varphi^{*},
$$

where $\varphi^{*}$ is the restriction of scalars functor. We can verify that $\varphi^{*}$ preserves fibrations and weak equivalences. Hence the pair $\left(B \otimes_{A}(-), \varphi^{*}\right)$ is a Quillen adjunction. We have natural isomorphisms $B \otimes_{A}(M \otimes K) \cong\left(B \otimes_{A} M\right) \otimes K$ in $\operatorname{Mod}_{B}(\mathbf{M})$ for $M \in \operatorname{Mod}_{A}(\mathbf{M})$ and $K \in \Sigma \mathrm{Sp}$ which reduce to the canonical isomorphism when $K$ is the sphere spectrum, and are compatible with the associativity isomorphisms in $\operatorname{Mod}_{A}(\mathbf{M})$ and $\operatorname{Mod}_{B}(\mathbf{M})$ with respect to the tensor structures over $\Sigma$ Sp. Hence we obtain the following lemma.

Lemma 4.1 The adjoint pair $\left(B \otimes_{A}(-), \varphi^{*}\right)$ is a $\Sigma \mathrm{Sp}$-Quillen adjunction.

Now suppose that $A$ and $B$ are commutative monoid objects in $\mathbf{M}$ and $\varphi: A \rightarrow B$ is a map of commutative monoid objects. The left Quillen functor $B \otimes_{A}(-)$ : $\operatorname{Mod}_{A}(\mathbf{M}) \rightarrow \operatorname{Mod}_{B}(\mathbf{M})$ is a strong symmetric monoidal functor between the symmetric monoidal categories. Since $A$ is cofibrant in $\operatorname{Mod}_{A}(\mathbf{M})$, we see that $B \otimes_{A}(-)$ is a symmetric monoidal left Quillen functor. Furthermore, $B \otimes_{A}(-)$ is a symmetric monoidal left $\Sigma$ Sp-Quillen functor since there are natural isomorphisms $B \otimes_{A}(A \otimes K) \cong B \otimes K$ for $K \in \Sigma$ Sp. Hence we obtain the following lemma.

Lemma 4.2 If $A$ and $B$ are commutative monoid objects in $\mathbf{M}$ and $\varphi: A \rightarrow B$ is a map of commutative monoid objects, then the pair $\left(B \otimes_{A}(-), \varphi^{*}\right)$ is a symmetric monoidal $\Sigma \mathrm{Sp}-$ Quillen adjunction.

\subsection{Embeddings of module categories}

In this subsection we formulate embeddings of module categories in combinatorial symmetric monoidal $\Sigma$ Sp-model categories satisfying the monoid axiom. We show 
that a certain full subcategory of a category of module objects can be embedded into another category of module objects as an enriched category over the stable homotopy category of spectra.

First, we formulate the setting. Let $\mathbf{M}$ and $\mathbf{N}$ be combinatorial symmetric monoidal $\Sigma$ Sp-model categories satisfying the monoid axiom. We suppose that we have a symmetric monoidal $\Sigma$ Sp-Quillen adjunction $i: \mathbf{M} \rightleftarrows \mathbf{N}: j$. We take monoid objects $A$ in $\mathbf{M}$ and $B$ in $\mathbf{N}$. We suppose that there is a morphism of monoid objects

$$
\varphi: i(A) \longrightarrow B
$$

By Lemma 4.1, the morphism $\varphi$ induces a $\Sigma$ Sp-Quillen adjunction $\left(B \otimes_{i(A)}(-), \varphi^{*}\right)$. Since $i$ is strong symmetric monoidal, it induces a functor $i: \operatorname{Mod}_{A}(\mathbf{M}) \rightarrow$ $\operatorname{Mod}_{i(A)}(\mathbf{N})$. We see that $j$ induces a right adjoint $j: \operatorname{Mod}_{i(A)}(\mathbf{N}) \rightarrow \operatorname{Mod}_{A}(\mathbf{M})$ to $i$. Composing these two adjunctions, we obtain an adjoint pair of functors

$$
\operatorname{Ex}: \operatorname{Mod}_{A}(\mathbf{M}) \rightleftarrows \operatorname{Mod}_{B}(\mathbf{N}): \operatorname{Re} .
$$

Lemma 4.3 The pair (Ex, $\mathrm{Re})$ is a $\Sigma \mathrm{Sp}$-Quillen adjunction.

Proof By definition of the model structures, $\varphi^{*}$ and $j$ are right Quillen functors. Hence the composition $\operatorname{Re}=j \circ \varphi^{*}$ is also a right Quillen functor. Since the pair $(i, j)$ is a symmetric monoidal $\Sigma$ Sp-Quillen adjunction, we have natural isomorphisms $i(M \otimes K) \cong i(M) \otimes K$ for $M \in \mathbf{M}$ and $K \in \Sigma S$. These isomorphisms induce natural isomorphisms $\operatorname{Ex}(M \otimes K) \cong \operatorname{Ex}(M) \otimes K$ for $M \in \operatorname{Mod}_{A}(\mathbf{M})$ and $K \in \Sigma$ Sp which reduce to the canonical isomorphism when $K$ is the sphere spectrum, and are compatible with the associativity isomorphisms in $\operatorname{Mod}_{A}(\mathbf{M})$ and $\operatorname{Mod}_{B}(\mathbf{N})$ with respect to the tensor structures over $\Sigma$ Sp. This completes the proof.

The homotopy categories $\mathrm{Ho}\left(\operatorname{Mod}_{A}(\mathbf{M})\right)$ and $\mathrm{Ho}\left(\operatorname{Mod}_{B}(\mathbf{N})\right)$ are closed $\mathrm{Ho}(\Sigma \mathrm{Sp})$ modules by [20, Thm. 4.3.4]. Furthermore, $\operatorname{Ho}\left(\operatorname{Mod}_{A}(\mathbf{M})\right)$ and $\operatorname{Ho}\left(\operatorname{Mod}_{B}(\mathbf{N})\right)$ are triangulated categories since $\operatorname{Mod}_{A}(\mathbf{M})$ and $\operatorname{Mod}_{B}(\mathbf{N})$ are stable model categories by [36, Lem. 3.5.2]. By Lemma 4.3, we obtain an adjunction of closed $\mathrm{Ho}(\Sigma \mathrm{Sp})$-modules

$$
\mathbb{L E x}: \operatorname{Ho}\left(\operatorname{Mod}_{A}(\mathbf{M})\right) \rightleftarrows \operatorname{Ho}\left(\operatorname{Mod}_{B}(\mathbf{N})\right): \mathbb{R R e},
$$

where $\mathbb{L E x}$ is the total left derived functor of Ex and $\mathbb{R} R$ is the total right derived functor of Re. Note that $\mathbb{L E x}$ and $\mathbb{R} R$ e are exact functors between the triangulated categories.

Now we consider the case where $A \in \mathbf{M}$ and $B \in \mathbf{N}$ are commutative monoid objects and $\varphi: i(A) \rightarrow B$ is a map of commutative monoid objects. In this case $\operatorname{Mod}_{A}(\mathbf{M})$ and $\operatorname{Mod}_{B}(\mathbf{N})$ are symmetric monoidal $\Sigma$ Sp-model categories. Since the left adjoint Ex of the Quillen adjunction Ex : $\operatorname{Mod}_{A}(\mathbf{M}) \rightleftarrows \operatorname{Mod}_{B}(\mathbf{N}): \operatorname{Re}$ is strong symmetric monoidal, the pair (Ex, Re) is a symmetric monoidal Quillen adjunction. Furthermore, since there are natural isomorphisms $B \otimes_{i(A)} i(A \otimes K) \cong B \otimes K$ for $K \in \Sigma$ Sp, we obtain the following lemma. 
Lemma 4.4 If $A \in \mathbf{M}$ and $B \in \mathbf{N}$ are commutative monoid objects and $\varphi: i(A) \rightarrow$ $B$ is a map of commutative monoid objects, then the pair (Ex, $\mathrm{Re})$ is a symmetric monoidal $\Sigma \mathrm{Sp}-Q u i l l e n$ adjunction.

If $A$ and $B$ are commutative monoid objects, then the homotopy categories $\mathrm{Ho}\left(\operatorname{Mod}_{A}(\mathbf{M})\right)$ and $\mathrm{Ho}\left(\operatorname{Mod}_{B}(\mathbf{N})\right)$ are closed symmetric monoidal Ho( $(\Sigma \mathrm{Sp})$-algebras (see [20, §4.2]). Furthermore, if $\varphi: i(A) \rightarrow B$ is a map of commutative monoid objects, then the induced pair ( $\mathbb{L E x}, \mathbb{R R e}$ ) of functors is an adjunction of symmetric monoidal Ho( $\Sigma$ Sp)-algebras (see the second paragraph of Sect. 4.1 for the definition of these concepts).

Now suppose $A$ and $B$ are monoid objects and $\varphi: i(A) \rightarrow B$ is a map of monoid objects. We shall define a full subcategory $\mathbf{T}$ of $\operatorname{Ho}\left(\operatorname{Mod}_{A}(\mathbf{M})\right)$ and show that $\mathbf{T}$ can be embedded into $\mathrm{Ho}\left(\operatorname{Mod}_{B}(\mathbf{N})\right)$ as an $\mathrm{Ho}(\Sigma \mathrm{Sp})$-enriched category through the functor $\mathbb{L E x}$. Let $\mathbf{T}$ be the full subcategory of $\operatorname{Ho}\left(\operatorname{Mod}_{A}(\mathbf{M})\right)$ consisting of $X$ such that the unit map $X \rightarrow \mathbb{R} \operatorname{ReLEx}(X)$ is an isomorphism

$$
\mathbf{T}=\left\{X \in \operatorname{Ho}\left(\operatorname{Mod}_{A}(\mathbf{M})\right) \mid X \stackrel{\cong}{\longrightarrow} \operatorname{ReLE} \operatorname{Ex}(X)\right\}
$$

It is easy to see that $\mathbf{T}$ is a thick subcategory of $\operatorname{Ho}\left(\operatorname{Mod}_{A}(\mathbf{M})\right)$.

Proposition 4.5 The restriction of $\mathbb{L} E x$ to $\mathbf{T}$ is fully faithful as an $\mathrm{Ho}(\Sigma \mathrm{Sp})$-enriched functor.

Proof This follows from the natural isomorphism

$$
\mathbb{R M a p}_{\operatorname{Mod}_{B}(\mathbf{N})}(\mathbb{L E x}(X), \mathbb{L E x}(Y)) \cong \mathbb{R M a p}_{\operatorname{Mod}_{A}(\mathbf{M})}(X, \mathbb{R} \operatorname{Re} \mathbb{L E x}(Y))
$$

in $\operatorname{Ho}(\Sigma \mathrm{Sp})$, where $\mathbb{R} \operatorname{Map}_{\operatorname{Mod}_{A}(\mathbf{M})}(-,-)$ and $\mathbb{R} \operatorname{Map}_{\operatorname{Mod}_{B}(\mathbf{N})}(-,-)$ are derived mapping spaces of $\operatorname{Mod}_{A}(\mathbf{M})$ and $\operatorname{Mod}_{B}(\mathbf{N})$, respectively.

\subsection{Homotopy fixed points functor}

In this subsection we shall discuss the relationship between the homotopy fixed points functors and the embeddings considered in Sect. 4.2. We show that the full subcategory $\mathbf{T}$ contains all dualizable objects in some appropriate settings.

First, we recall the definition of homotopy fixed points functors (see $[4, \S \S 3$ and 5]). By Proposition 3.9, we have a symmetric monoidal $\Sigma S p$-Quillen adjunction

$$
\operatorname{triv}: \Sigma \operatorname{Sp}_{k} \rightleftarrows \Sigma \operatorname{Sp}(G)_{k}:(-)^{G} \text {. }
$$

Let $A$ be a monoid object in $\Sigma \operatorname{Sp}_{k}$ and we regard $A$ as a monoid object in $\Sigma \operatorname{Sp}(G)_{k}$ with trivial $G$-action. Let $\varphi: A \rightarrow B$ be a map of monoid objects in $\Sigma \operatorname{Sp}(G)_{k}$. By Lemma 4.3, we have a $\Sigma$ Sp-Quillen adjunction

$$
\operatorname{Ex}: \operatorname{Mod}_{A}\left(\Sigma \operatorname{Sp}_{k}\right) \rightleftarrows \operatorname{Mod}_{B}\left(\Sigma \operatorname{Sp}(G)_{k}\right): \operatorname{Re} .
$$


This induces an adjunction

$$
\mathbb{L E x}: \operatorname{Ho}\left(\operatorname{Mod}_{A}\left(\Sigma \operatorname{Sp}_{k}\right)\right) \rightleftarrows \operatorname{Ho}\left(\operatorname{Mod}_{B}\left(\Sigma \operatorname{Sp}(G)_{k}\right)\right): \mathbb{R} \operatorname{Re}
$$

of closed $\operatorname{Ho}(\Sigma \mathrm{Sp})$-modules. We define a homotopy fixed points functor $(-)^{h G}$ to be the total right derived functor of Re:

$$
(-)^{h G}=\mathbb{R R e}: \operatorname{Ho}\left(\operatorname{Mod}_{B}\left(\Sigma \operatorname{Sp}(G)_{k}\right)\right) \longrightarrow \operatorname{Ho}\left(\operatorname{Mod}_{A}\left(\Sigma \operatorname{Sp}_{k}\right)\right) \text {. }
$$

Although the definition of the homotopy fixed points spectrum $X^{h G}$ depends on the map $\varphi$, we shall show that the homotopy type of the underlying spectrum of $X^{h G}$ is independent of $\varphi$ and also agrees with the underlying notion of homotopy fixed points on $\Sigma \operatorname{Sp}(G)_{k}$. There is a diagram

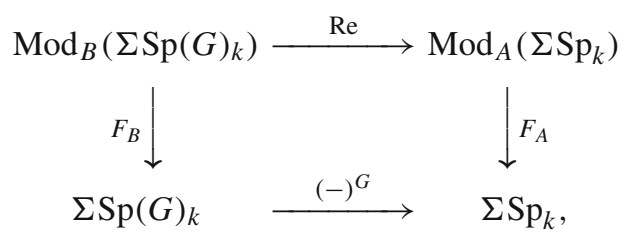

where $F_{A}: \operatorname{Mod}_{A}\left(\Sigma \operatorname{Sp}_{k}\right) \rightarrow \Sigma \operatorname{Sp}_{k}$ and $F_{B}: \operatorname{Mod}_{B}\left(\Sigma \operatorname{Sp}(G)_{k}\right) \rightarrow \Sigma \operatorname{Sp}(G)_{k}$ are forgetful functors. This diagram is commutative on the nose since the functor $\operatorname{Re}: \operatorname{Mod}_{B}\left(\Sigma \operatorname{Sp}(G)_{k}\right) \rightarrow \operatorname{Mod}_{A}\left(\Sigma \operatorname{Sp}_{k}\right)$ is the composition $(-)^{G} \circ \varphi^{*}$, where $\varphi^{*}: \operatorname{Mod}_{B}\left(\Sigma \operatorname{Sp}(G)_{k}\right) \rightarrow \operatorname{Mod}_{A}\left(\Sigma \operatorname{Sp}(G)_{k}\right)$ is the restriction of scalars functor and $(-)^{G}: \operatorname{Mod}_{A}\left(\Sigma \operatorname{Sp}(G)_{k}\right) \rightarrow \operatorname{Mod}_{A}\left(\Sigma \operatorname{Sp}_{k}\right)$ is the fixed points functor on $\operatorname{Mod}_{A}\left(\Sigma \operatorname{Sp}(G)_{k}\right)$ induced by the fixed points functor on $\Sigma \operatorname{Sp}(G)_{k}$. Since $F_{A}$ and $F_{B}$ preserve weak equivalences, they induce functors $h F_{A}: \operatorname{Ho}\left(\operatorname{Mod}_{A}\left(\Sigma \operatorname{Sp}_{k}\right)\right) \rightarrow$ $\operatorname{Ho}\left(\Sigma \operatorname{Sp}_{k}\right)$ and $h F_{B}: \operatorname{Ho}\left(\operatorname{Mod}_{B}\left(\Sigma \operatorname{Sp}(G)_{k}\right)\right) \rightarrow \operatorname{Ho}\left(\Sigma \operatorname{Sp}(G)_{k}\right)$ between the homotopy categories, respectively. Furthermore, since $F_{A}$ and $F_{B}$ preserve fibrations, we obtain a natural isomorphism

$$
h F_{A}\left(X^{h G}\right) \stackrel{\cong}{\longrightarrow}\left(h F_{B} X\right)^{h G}
$$

in $\operatorname{Ho}\left(\Sigma \operatorname{Sp}_{k}\right)$ for any $X \in \operatorname{Ho}\left(\operatorname{Mod}_{B}\left(\Sigma \operatorname{Sp}(G)_{k}\right)\right)$. This means the homotopy type of the underlying spectrum of $X^{h G}$ is independent of $\varphi$ and also agrees with the underlying notion of homotopy fixed points on $\Sigma \operatorname{Sp}(G)_{k}$.

Since $A$ is cofibrant in $\operatorname{Mod}_{A}\left(\Sigma \operatorname{Sp}_{k}\right)$, we have an isomorphism $\mathbb{L E x}(A) \cong B$. Hence the unit of the adjunction $\left(\mathbb{L E x}, \mathbb{R} R\right.$ ) gives a map $A \rightarrow B^{h G}$ in $\operatorname{Ho}\left(\operatorname{Mod}_{A}\left(\Sigma \operatorname{Sp}_{k}\right)\right)$. Recall that $\mathbf{T}$ is the full subcategory of $\operatorname{Ho}\left(\operatorname{Mod}_{A}\left(\Sigma \operatorname{Sp}_{k}\right)\right)$ consisting of $X$ such that the unit map $X \rightarrow \mathbb{R} \operatorname{Re} L E x(X)$ is an isomorphism. Hence $A \in \mathbf{T}$ if and only if the map $A \rightarrow B^{h G}$ is an isomorphism.

Now we suppose $A \in \Sigma \operatorname{Sp}_{k}$ and $B \in \Sigma \operatorname{Sp}(G)_{k}$ are commutative monoid objects and $\varphi: A \rightarrow B$ is a map of commutative monoid objects. In this case the pair $\mathbb{L E x}: \operatorname{Ho}\left(\operatorname{Mod}_{A}\left(\Sigma \operatorname{Sp}_{k}\right)\right) \rightleftarrows \operatorname{Ho}\left(\operatorname{Mod}_{B}\left(\Sigma \operatorname{Sp}(G)_{k}\right)\right): \mathbb{R R e}$ of functors is an adjunction of symmetric monoidal $\operatorname{Ho}(\Sigma \mathrm{Sp})$-algebras. In particular, $\operatorname{Ho}\left(\operatorname{Mod}_{A}\left(\Sigma \operatorname{Sp}_{k}\right)\right)$ 
and $\operatorname{Ho}\left(\operatorname{Mod}_{B}(\Sigma \operatorname{Sp}(G))_{k}\right)$ are symmetric monoidal categories and $\mathbb{L E x}$ is a strong symmetric monoidal functor. We show that $\mathbf{T}$ contains all dualizable objects in $\operatorname{Ho}\left(\operatorname{Mod}_{A}\left(\Sigma \mathrm{Sp}_{k}\right)\right)$ if $A \in \mathbf{T}$.

Proposition 4.6 We assume that $A \in \Sigma \operatorname{Sp}_{k}$ and $B \in \Sigma \operatorname{Sp}(G)_{k}$ are commutative monoid objects and $\varphi: A \rightarrow B$ is a map of commutative monoid objects. If $A \rightarrow B^{h G}$ is an isomorphism, then $\mathbf{T}$ contains all dualizable objects in $\operatorname{Ho}\left(\operatorname{Mod}_{A}\left(\Sigma \operatorname{Sp}_{k}\right)\right)$.

Proof We put $\mathbf{M}=\operatorname{Mod}_{A}\left(\Sigma \operatorname{Sp}_{k}\right)$ and $\mathbf{N}=\operatorname{Mod}_{B}\left(\Sigma \operatorname{Sp}(G)_{k}\right)$. The homotopy categories $\mathrm{Ho}(\mathbf{M})$ and $\mathrm{Ho}(\mathbf{N})$ are symmetric monoidal categories with tensor products $\otimes_{A}^{\mathbb{L}}$ and $\otimes_{B}^{\mathbb{L}}$, respectively. We let $X$ be a dualizable object in $\operatorname{Ho}(\mathbf{M})$ and denote by $D X$ its dual. Since $\mathbb{L E x}$ is strong symmetric monoidal and $\mathbb{L E x}(A) \cong B, \mathbb{L E x}(X)$ is a dualizable object in $\operatorname{Ho}(\mathbf{N})$ and its dual is $\mathbb{L} \operatorname{Ex}(D X)$. For any $W \in \operatorname{Ho}(\mathbf{M})$, we have a natural isomorphism

$$
[W, \mathbb{R} \operatorname{Re} \mathbb{L E x}(X)]_{\mathbf{M}} \cong\left[\mathbb{L} \operatorname{Ex}(W) \otimes_{B}^{\mathbb{L}} \mathbb{L} \operatorname{Ex}(D X), \mathbb{L E x}(A)\right]_{\mathbf{N}} .
$$

We have $\mathbb{L} \operatorname{Ex}(W) \otimes_{B}^{\mathbb{L}} \mathbb{L} \operatorname{Ex}(D X) \cong \mathbb{L E x}\left(W \otimes_{A}^{\mathbb{L}} D X\right)$. This implies the following isomorphism

$$
\left[\mathbb{L E x}(W) \otimes_{B}^{\mathbb{L}} \mathbb{L E x}(D X), \mathbb{L} \operatorname{Ex}(A)\right]_{\mathbf{N}} \cong\left[W, \mathbb{R} \operatorname{Re} \mathbb{L E x}(A) \otimes_{A}^{\mathbb{L}} X\right]_{\mathbf{M}} .
$$

By the Yoneda lemma, we obtain $\mathbb{R} \operatorname{Re} L \operatorname{Ex}(X) \cong \mathbb{R} \operatorname{Re} \mathbb{L E x}(A) \otimes_{A}^{\mathbb{L}} X$. By the assump-

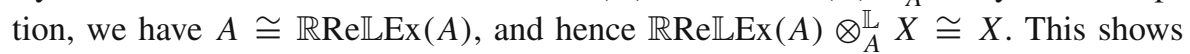
$X \cong \mathbb{R R e L E x}(X)$.

Remark 4.7 We note that $A \rightarrow B^{h G}$ is an isomorphism under some conditions if $B$ is a consistent $k$-local $G$-Galois extension.

First, we recall the definition of $k$-local Amitsur derived completion (see, for example, [34, Def. 8.2.1]). Let $A$ be a $k$-local cofibrant commutative symmetric ring spectrum. For a cofibrant commutative $A$-algebra $C$, the $k$-local Amitsur derived completion $A_{k, C}^{\wedge}$ is the homotopy limit of the cosimplicial spectrum $L_{k} C^{\wedge_{A} \bullet+1}$ given by

$$
L_{k} C^{\wedge_{A} n+1}=L_{k}(\overbrace{C \wedge_{A} \cdots \wedge_{A} C}^{n+1})
$$

with the usual cosimplicial structure, where $L_{k}$ is the localization functor with respect to $k$.

The $k$-local $G$-Galois extension $\varphi: A \rightarrow B$ in the sense of [4, Def. 6.2.1] is said to be consistent if the coaugmentation of the $k$-local Amitsur derived completion $A \rightarrow A_{k, B}^{\wedge}$ is an equivalence (see [4, Def. 1.0.4(1)]).

We suppose the localization functor $L_{k}$ is given as a composite of two localization functors $L_{M} L_{T}$, where $L_{T}$ is a smashing localization and $L_{M}$ is a localization with respect to a finite spectrum $M$ (cf. [4, Assumption 1.0.3]). Furthermore, we suppose $G$ has finite virtual cohomological dimension. If $\varphi: A \rightarrow B$ is a consistent $k$-local $G$-Galois extension, then $\varphi$ induces an isomorphism $A \stackrel{\cong}{\rightrightarrows} B^{h G}$ by [4, Prop. 6.1.7(3) and Cor. 6.3.2]. 


\subsection{Finite Galois extensions}

In this subsection, as an example, we consider the case associated to a finite Galois extension of symmetric spectra. Now suppose that $G$ is a finite group and $k$ is an arbitrary symmetric spectrum. We assume that $\varphi: A \rightarrow B$ is a faithful $k$-local $G$ Galois extension of symmetric spectra (see [34, Ch. 4]). We have a symmetric monoidal $\Sigma$ Sp-Quillen adjunction Ex : $\operatorname{Mod}_{A}\left(\Sigma \operatorname{Sp}_{k}\right) \rightleftarrows \operatorname{Mod}_{B}\left(\Sigma \operatorname{Sp}(G)_{k}\right): \operatorname{Re}$ and its derived adjunction $\mathbb{L E x}: \operatorname{Ho}\left(\operatorname{Mod}_{A}\left(\Sigma \operatorname{Sp}_{k}\right)\right) \rightleftarrows \operatorname{Ho}\left(\operatorname{Mod}_{B}\left(\Sigma \operatorname{Sp}(G)_{k}\right)\right): \mathbb{R R e}$. In this case we shall show that the full subcategory $\mathbf{T}$ is the whole category $\operatorname{Ho}\left(\operatorname{Mod}_{A}\left(\Sigma \operatorname{Sp}_{k}\right)\right)$, where T consists of objects $X$ in $\operatorname{Ho}\left(\operatorname{Mod}_{A}\left(\Sigma \operatorname{Sp}_{k}\right)\right)$ such that the unit map $X \rightarrow \mathbb{R R e L E x}(X)$ is an isomorphism. Furthermore, we shall show that the adjoint pair (Ex, Re) is a Quillen equivalence.

First, we recall that an $A$-module object $M$ is said to be $k$-locally faithful if $M \wedge_{A}$ $N \cong 0$ implies $N \cong 0$ in $\operatorname{Ho}\left(\operatorname{Mod}_{A}\left(\Sigma \operatorname{Sp}_{k}\right)\right)$. A $k$-local $G$-Galois extension $\varphi: A \rightarrow B$ is said to be faithful if $U B$ is a $k$-locally faithful $A$-module, where $U: \Sigma \operatorname{Sp}(G)_{k} \rightarrow$ $\Sigma \mathrm{Sp}_{k}$ is the forgetful functor. By [34, Prop. 6.2.1], if $\varphi: A \rightarrow B$ is a $k$-local $G$-Galois extension, then $U B$ is a dualizable object in $\operatorname{Ho}\left(\operatorname{Mod}_{A}\left(\Sigma \operatorname{Sp}_{k}\right)\right)$.

For any $N \in \operatorname{Ho}\left(\operatorname{Mod}_{A}\left(\Sigma \operatorname{Sp}_{k}\right)\right)$, we have the unit map $N \rightarrow \mathbb{R R e} \mathbb{L E x}(N)$. Since $\mathbb{R R e}=(-)^{h G}$ is the homotopy fixed points functor, we can identify this map with the map $N \rightarrow\left(B \wedge_{A} N\right)^{h G}$. We show that the map $N \rightarrow\left(B \wedge_{A} N\right)^{h G}$ is an isomorphism for any $N \in \operatorname{Ho}\left(\operatorname{Mod}_{A}\left(\Sigma \operatorname{Sp}_{k}\right)\right)$. This means the full subcategory $\mathbf{T}$ is the whole category $\operatorname{Ho}\left(\operatorname{Mod}_{A}\left(\Sigma \mathrm{Sp}_{k}\right)\right)$.

Lemma 4.8 Suppose that $G$ is a finite group and $\varphi: A \rightarrow B$ is a faithful $k$-local $G$-Galois extension. The map $N \rightarrow\left(B \wedge_{A} N\right)^{h G}$ is an isomorphism for any $N \in$ $\operatorname{Ho}\left(\operatorname{Mod}_{A}\left(\Sigma \operatorname{Sp}_{k}\right)\right)$, and hence $\mathbf{T}=\operatorname{Ho}\left(\operatorname{Mod}_{A}\left(\Sigma \operatorname{Sp}_{k}\right)\right)$.

Proof Since $U B$ is $k$-locally faithful over $A$, it suffices to show that the map $U B \wedge_{A}$ $N \rightarrow U B \wedge_{A}\left(B \wedge_{A} N\right)^{h G}$ is an isomorphism in $\operatorname{Ho}\left(\operatorname{Mod}_{A}\left(\Sigma \operatorname{Sp}_{k}\right)\right)$. Since $U B$ is a dualizable object, we have an isomorphism $U B \wedge_{A}\left(B \wedge_{A} N\right)^{h G} \cong\left(U B \wedge_{A} B \wedge_{A}\right.$ $N)^{h G}$ in $\operatorname{Ho}\left(\operatorname{Mod}_{A}\left(\Sigma \operatorname{Sp}_{k}\right)\right)$. By the assumption that $\varphi: A \rightarrow B$ is a $k$-local $G$ Galois extension, we have an equivalence $U B \wedge_{A} B \simeq \operatorname{Map}(G, U B)$. This induces an equivalence $U B \wedge_{A} B \wedge_{A} N \simeq \operatorname{Map}\left(G, U B \wedge_{A}, N\right)$. Hence we see that $\left(U B \wedge_{A}\right.$ $\left.B \wedge_{A} N\right)^{h G} \simeq U B \wedge_{A} N$ and the map $U B \wedge_{A} N \rightarrow U B \wedge_{A}\left(B \wedge_{A} N\right)^{h G}$ is an isomorphism in $\operatorname{Ho}\left(\operatorname{Mod}_{A}\left(\Sigma \operatorname{Sp}_{k}\right)\right)$. This completes the proof.

Next, we would like to show that the adjunction $(\mathrm{Ex}, \mathrm{Re})$ is actually a Quillen equivalence. In order to show that the following lemma is useful.

Lemma 4.9 Suppose that $G$ is a finite group and $\varphi: A \rightarrow B$ is a faithful $k$-local $G$-Galois extension. For any $Z \in \operatorname{Ho}\left(\operatorname{Mod}_{B}\left(\Sigma \operatorname{Sp}(G)_{k}\right)\right)$, if $Z^{h G}$ is trivial, then $Z$ is trivial.

Proof We may assume that $Z$ is an object in $\operatorname{Mod}_{B}\left(\Sigma \operatorname{Sp}(G)_{k}\right)$ that is both fibrant and cofibrant. We have a map $U B \wedge_{A} Z \rightarrow \operatorname{Map}(G, U Z)$ in $\Sigma \operatorname{Sp}(G)_{k}$, which is the adjoint of the $U B$-action map $U\left(U B \wedge_{A} Z\right) \cong U B \wedge_{A} U Z \rightarrow U Z$ of $U Z$. We shall show that this map is a weak equivalence. For this purpose, it suffices to 
show that $U B \wedge_{A} U Z \rightarrow U \operatorname{Map}(G, U Z)$ is a weak equivalence. Using the fact that $U B \wedge_{A} U B \simeq U \operatorname{Map}(G, U B)$, we see that $U B \wedge_{A} U Z \simeq U \operatorname{Map}(G, U Z)$ and the map $U B \wedge_{A} U Z \rightarrow U \operatorname{Map}(G, U Z)$ is a weak equivalence.

The weak equivalence $U B \wedge_{A} Z \rightarrow \operatorname{Map}(G, U Z)$ implies that $\left(U B \wedge_{A} Z\right)^{h G \cong}$ $\operatorname{Map}(G, U Z)^{h G}$ in $\operatorname{Ho}\left(\operatorname{Mod}_{A}\left(\Sigma \operatorname{Sp}_{k}\right)\right)$. Since $U B$ is a dualizable object, we have

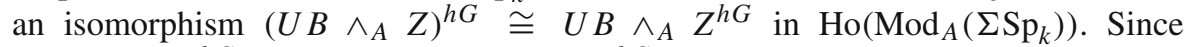
$\operatorname{Map}(G, U Z)^{h G} \cong U Z$, we see that, if $Z^{h G}$ is trivial, $U Z$ is trivial and hence $Z$ is trivial.

Using this lemma, we obtain the following proposition which says the adjunction $(\mathrm{Ex}, \mathrm{Re})$ is a Quillen equivalence if it is associated to a faithful $k$-local finite Galois extension.

Proposition 4.10 If $G$ is a finite group and $\varphi: A \rightarrow B$ is a faithful $k$-local $G$-Galois extension, then the adjoint pair

$$
\operatorname{Ex}: \operatorname{Mod}_{A}\left(\Sigma \operatorname{Sp}_{k}\right) \rightleftarrows \operatorname{Mod}_{B}\left(\Sigma \operatorname{Sp}(G)_{k}\right): \operatorname{Re}
$$

is a Quillen equivalence.

Proof Let $X$ be a cofibrant object in $\operatorname{Mod}_{A}\left(\Sigma \mathrm{Sp}_{k}\right)$ and $Y$ a fibrant object in $\operatorname{Mod}_{B}\left(\Sigma \operatorname{Sp}(G)_{k}\right)$. Suppose that $f: B \wedge_{A} X \rightarrow Y$ is a weak equivalence in $\operatorname{Mod}_{B}\left(\Sigma \operatorname{Sp}(G)_{k}\right)$. Then we can regard $Y$ as a fibrant replacement of $B \wedge_{A} X$ and hence $Y^{G}$ represents $\left(B \wedge_{A} X\right)^{h G}$ in $\operatorname{Ho}\left(\operatorname{Mod}_{A}\left(\Sigma \operatorname{Sp}_{k}\right)\right)$. By Lemma 4.8, the adjoint map $g: X \rightarrow Y^{G}$ to $f$ induces an isomorphism $X \stackrel{\cong}{\rightarrow}\left(B \wedge_{A} X\right)^{h G}$ in the homotopy category $\operatorname{Ho}\left(\operatorname{Mod}_{A}\left(\Sigma \operatorname{Sp}_{k}\right)\right)$ and hence the map $g: X \rightarrow Y^{G}$ is a weak equivalence.

Conversely, suppose that $g: X \rightarrow Y^{G}$ is a weak equivalence in $\operatorname{Mod}_{A}\left(\Sigma \operatorname{Sp}_{k}\right)$. We have to show that the adjoint map $f: B \wedge_{A} X \rightarrow Y$ to $g$ is a weak equivalence. We let $Z$ be the cofiber of the map $f$ in $\operatorname{Ho}\left(\operatorname{Mod}_{B}\left(\Sigma \operatorname{Sp}(G)_{k}\right)\right)$. Note that $Y^{h G}$ is represented

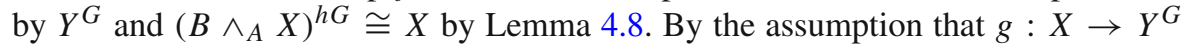
is a weak equivalence, we see that the induced map $f^{h G}:\left(B \wedge_{A} X\right)^{h G} \rightarrow Y^{h G}$ is an isomorphism in $\operatorname{Ho}\left(\operatorname{Mod}_{A}\left(\Sigma \operatorname{Sp}_{k}\right)\right)$, and hence $Z^{h G}$ is trivial. By Lemma 4.9, $Z$ is trivial and hence $f$ is an isomorphism in $\operatorname{Ho}\left(\operatorname{Mod}_{B}\left(\Sigma \operatorname{Sp}(G)_{k}\right)\right)$. This shows that $f: B \wedge_{A} X \rightarrow Y$ is a weak equivalence. This completes the proof.

\subsection{The $K(n)$-local category}

In this subsection we shall apply the results in Sects. 4.2 and 4.3 to the $K(n)$-local category. Let $E_{n}$ be the $n$th Morava $E$-theory spectrum and $K(n)$ the $n$th Morava $K$ theory spectrum at a prime $p$. We denote by $\mathbb{G}_{n}$ the extended Morava stabilizer group. In this subsection we shall show that the $K(n)$-local category can be embedded as an enriched category over the stable homotopy category of spectra into the homotopy category of module objects over a discrete model $F_{n}$ of $E_{n}$ in the category of $K(n)$ local discrete symmetric $\mathbb{G}_{n}$-spectra.

In [9, Def. 2.3] Davis constructed a discrete $\mathbb{G}_{n}$-spectrum $F_{n}$ by using results in [11]. We shall review the construction of $F_{n}$. Devinatz-Hopkins [11] gave a functorial construction of commutative $S$-algebras $E_{n}^{d h U}$ for open subgroups $U$ of $\mathbb{G}_{n}$ which 
satisfy the desired properties of the homotopy fixed points spectra. The spectrum $F_{n}$ is defined by

$$
F_{n}=\underset{U}{\operatorname{colim}} E_{n}^{d h U}
$$

where the colimit is taken over the open subgroups $U$ of $\mathbb{G}_{n}$. Note that $F_{n}$ is $E(n)$-local, where $E(n)$ is the $n$th Johnson-Wilson spectrum at $p$, since the Bousfield localization $L_{E(n)}$ with respect to $E(n)$ is smashing and $F_{n}$ is the colimit of the $E(n)$-local spectra $E_{n}^{d h U}$, and that the spectrum $E_{n}$ is recovered by the equivalence

$$
E_{n} \simeq L_{K(n)} F_{n}
$$

in the stable homotopy category of spectra by [9, Thm 6.3], where $L_{K(n)}$ is the Bousfield localization with respect to $K(n)$.

In $[4, \S 8.1]$ Behrens-Davis showed that $F_{n}$ can be taken as a commutative monoid object in $\Sigma \operatorname{Sp}\left(\mathbb{G}_{n}\right)$. Furthermore, they showed that $F_{n}$ is a consistent $K(n)$-local $\mathbb{G}_{n}$-Galois extension of $L_{K(n)} S$, where $S$ is the sphere spectrum. Note the $K(n)$ localization functor $L_{K(n)}$ is the composite $L_{F(n)} L_{E(n)}$ of two localization functors, where $L_{E(n)}$ is smashing and $L_{F(n)}$ is the localization functor with respect to any finite spectrum $F(n)$ of type $n$ at $p$. By Remark 4.7, this implies that the unit map $\varphi: S \rightarrow F_{n}$ induces an isomorphism $S \stackrel{\cong}{\rightarrow}\left(F_{n}\right)^{h \mathbb{G}_{n}}$ in $\operatorname{Ho}\left(\Sigma \operatorname{Sp}_{K(n)}\right)$ since $\mathbb{G}_{n}$ has finite virtual cohomological dimension.

We consider the adjunction

$$
\mathbb{L E x}: \operatorname{Ho}\left(\Sigma \operatorname{Sp}_{K(n)}\right) \rightleftarrows \operatorname{Ho}\left(\operatorname{Mod}_{F_{n}}\left(\Sigma \operatorname{Sp}\left(\mathbb{G}_{n}\right)_{K(n)}\right)\right): \mathbb{R R e}
$$

By Sect. 4.3, the right adjoint $\mathbb{R} R$ e is interpreted as the homotopy fixed points functor $(-)^{h \mathbb{G}_{n}}$. Hence the unit map of the adjunction is regarded as a map $X \rightarrow\left(\left(F_{n} \wedge\right.\right.$ $\left.X)_{K(n)}\right)^{h \mathbb{G}_{n}}$ for $X \in \operatorname{Ho}\left(\Sigma \operatorname{Sp}_{K(n)}\right)$, where $(-)_{K(n)}$ is the $K(n)$-localization functor on $\operatorname{Ho}\left(\operatorname{Mod}_{F_{n}}\left(\Sigma \operatorname{Sp}\left(\mathbb{G}_{n}\right)\right)\right.$.

Now we shall show that the homotopy fixed points functor $(-)^{h \mathbb{G}_{n}}$ is compatible with the $K(n)$-localization. We let $M$ be an object in $\operatorname{Ho}\left(\operatorname{Mod}_{F_{n}}\left(\Sigma \operatorname{Sp}\left(\mathbb{G}_{n}\right)\right)\right)$ and denote by $M_{K(n)}$ the $K(n)$-localization of $M$, which is represented by a fibrant replacement in $\operatorname{Mod}_{F_{n}}\left(\Sigma \operatorname{Sp}\left(\mathbb{G}_{n}\right)_{K(n)}\right)$ of a representative of $M$ in $\operatorname{Mod}_{F_{n}}\left(\Sigma \operatorname{Sp}\left(\mathbb{G}_{n}\right)\right)$. Since $\left(M_{K(n)}\right)^{h \mathbb{G}_{n}}$ is $K(n)$-local, we have a natural map

$$
L_{K(n)}\left(M^{h \mathbb{G}_{n}}\right) \longrightarrow\left(M_{K(n)}\right)^{h \mathbb{G}_{n}}
$$

in the stable homotopy category of spectra.

Lemma 4.11 The natural map $L_{K(n)}\left(M^{h \mathbb{G}_{n}}\right) \rightarrow\left(M_{K(n)}\right)^{h \mathbb{G}_{n}}$ is an isomorphism in the stable homotopy category of spectra for any object $M$ in $\operatorname{Ho}\left(\operatorname{Mod}_{F(n)}\left(\Sigma \operatorname{Sp}\left(\mathbb{G}_{n}\right)\right)\right)$.

Proof Let $F(n)$ be a finite spectrum of type $n$ at $p$. To show that the map $L_{K(n)}\left(M^{h \mathbb{G}_{n}}\right) \rightarrow\left(M_{K(n)}\right)^{h \mathbb{G}_{n}}$ is an isomorphism, it suffices to show that $F(n) \wedge$ 
$L_{K(n)}\left(M^{h \mathbb{G}_{n}}\right) \rightarrow F(n) \wedge\left(M_{K(n)}\right)^{h \mathbb{G}_{n}}$ is an isomorphism since $L_{K(n)}\left(M^{h \mathbb{G}_{n}}\right)$ and $\left(M_{K(n)}\right)^{h \mathbb{G}_{n}}$ are both $K(n)$-local.

First, note that $M$ is $E(n)$-local since $F_{n}$ is $E(n)$-local and $L_{E(n)}$ is smashing. Since $M$ and $M_{K(n)}$ are $E(n)$-local, the localization map $M \rightarrow M_{K(n)}$ induces an isomorphism $F(n) \wedge M \stackrel{\cong}{\rightarrow} F(n) \wedge M_{K(n)}$ in $\operatorname{Ho}\left(\operatorname{Mod}_{F_{n}}\left(\Sigma \operatorname{Sp}\left(\mathbb{G}_{n}\right)\right)\right.$. Hence a fibrant representative of $F(n) \wedge M$ in $\operatorname{Mod}_{F_{n}}\left(\Sigma \operatorname{Sp}\left(\mathbb{G}_{n}\right)\right)$ is also fibrant in $\operatorname{Mod}_{F_{n}}\left(\Sigma \operatorname{Sp}\left(\mathbb{G}_{n}\right)_{K(n)}\right)$. Therefore, we see that the homotopy fixed points spectrum $(F(n) \wedge M)^{h \mathbb{G}_{n}}$ taken on $\operatorname{Ho}\left(\operatorname{Mod}_{F_{n}}\left(\Sigma \operatorname{Sp}\left(\mathbb{G}_{n}\right)\right)\right)$ is isomorphic in the stable homotopy category of spectra to the homotopy fixed points spectrum $\left(F(n) \wedge M_{K(n)}\right)^{h \mathbb{G}_{n}}$ taken on $\operatorname{Ho}\left(\operatorname{Mod}_{F_{n}}\left(\Sigma \operatorname{Sp}\left(\mathbb{G}_{n}\right)\right)_{K(n)}\right)$.

Since $M$ is $E(n)$-local, $M^{h \mathbb{G}_{n}}$ is also $E(n)$-local. Using the facts that $F(n)$ is finite, $M^{h \mathbb{G}_{n}}$ is $E(n)$-local, and $L_{K(n)} \simeq L_{F(n)} L_{E(n)}$, we have a natural isomorphism $F(n) \wedge L_{K(n)}\left(M^{h \mathbb{G}_{n}}\right) \cong(F(n) \wedge M)^{h \mathbb{G}_{n}}$. On the other hand, since $F(n)$ is finite, we have a natural isomorphism $F(n) \wedge\left(M_{K(n)}\right)^{h \mathbb{G}_{n}} \cong\left(F(n) \wedge M_{K(n)}\right)^{h \mathbb{G}_{n}}$. Therefore, we see that the map $F(n) \wedge L_{K(n)}\left(M^{h \mathbb{G}_{n}}\right) \rightarrow F(n) \wedge\left(M_{K(n)}\right)^{h \mathbb{G}_{n}}$ is an isomorphism. This completes the proof.

By [9, Thm. 9.7] and [10, Thm. 1.1], the natural map $X \rightarrow L_{K(n)}\left(\left(F_{n} \wedge X\right)^{h \mathbb{G}_{n}}\right)$ is an isomorphism for any $X \in \operatorname{Ho}\left(\Sigma \mathrm{Sp}_{K(n)}\right)$. Using Lemma 4.11, we see that the unit map $X \rightarrow\left(\left(F_{n} \wedge X\right)_{K(n)}\right)^{h \mathbb{G}_{n}}$ is an isomorphism for any $X \in \operatorname{Ho}\left(\Sigma \operatorname{Sp}_{K(n)}\right)$. Hence we obtain the following theorem by Proposition 4.5.

Theorem 4.12 The functor

$$
\mathbb{L E x}: \operatorname{Ho}\left(\Sigma \operatorname{Sp}_{K(n)}\right) \longrightarrow \operatorname{Ho}\left(\operatorname{Mod}_{F_{n}}\left(\Sigma \operatorname{Sp}\left(\mathbb{G}_{n}\right)_{K(n)}\right)\right)
$$

is fully faithful as an $\mathrm{Ho}(\Sigma \mathrm{Sp})$-enriched functor.

The theory of localizations in enriched categories was developed by Wolff [40]. Theorem 4.12 implies that the $K(n)$-local category $\operatorname{Ho}\left(\Sigma \mathrm{Sp}_{K(n)}\right)$ is an $\operatorname{Ho}(\Sigma \mathrm{Sp})$ enriched coreflective subcategory of $\operatorname{Ho}\left(\operatorname{Mod}_{F_{n}}\left(\Sigma \operatorname{Sp}\left(\mathbb{G}_{n}\right)\right)_{K(n)}\right)$. By [40, Thm. 1.6], we obtain the following corollary.

Corollary 4.13 Let $\mathbf{W}$ be the class of morphisms $f$ in $\operatorname{Ho}\left(\operatorname{Mod}_{F_{n}}\left(\Sigma \operatorname{Sp}\left(\mathbb{G}_{n}\right)_{K(n)}\right)\right)$ such that the induced morphism $f^{h \mathbb{G}_{n}}=\mathbb{R} \operatorname{Re}(f)$ on homotopy fixed points spectra is an isomorphism in $\mathrm{Ho}\left(\Sigma \mathrm{Sp}_{K(n)}\right)$. The $K(n)$-local category $\mathrm{Ho}\left(\Sigma \mathrm{Sp}_{K(n)}\right)$ is equivalent to the localization of the homotopy category $\operatorname{Ho}\left(\operatorname{Mod}_{F_{n}}\left(\Sigma \operatorname{Sp}\left(\mathbb{G}_{n}\right)_{K(n)}\right)\right)$ with respect to $\mathbf{W}$ as an $\mathrm{Ho}(\Sigma \mathrm{Sp})$-enriched category

$$
\operatorname{Ho}\left(\Sigma \operatorname{Sp}_{K(n)}\right) \simeq \operatorname{Ho}\left(\operatorname{Mod}_{F_{n}}\left(\Sigma \operatorname{Sp}\left(\mathbb{G}_{n}\right)_{K(n)}\right)\right)\left[\mathbf{W}^{-1}\right]
$$

Remark 4.14 In the forthcoming paper [39] we will show that the symmetric monoidal $\Sigma$ Sp-Quillen adjunction Ex : $\Sigma \operatorname{Sp}_{K(n)} \rightleftarrows \operatorname{Mod}_{F_{n}}\left(\Sigma \operatorname{Sp}\left(\mathbb{G}_{n}\right)_{K(n)}\right):$ Re is a Quillen equivalence. 


\section{Embeddings of quasi-categories of modules over $\mathrm{Sp}_{k}$}

Let $\mathrm{Sp}_{k}$ be the underlying quasi-category of the simplicial model category $\Sigma \mathrm{Sp}_{k}$. Let $\psi: A \rightarrow E$ be a map of algebra objects in $\mathrm{Sp}_{k}$. We have an adjunction of underlying quasi-categories

$$
E \wedge_{A}(-): \operatorname{Mod}_{A}\left(\operatorname{Sp}_{k}\right) \rightleftarrows \operatorname{Mod}_{E}\left(\operatorname{Sp}_{k}\right): \psi^{*}
$$

In this section we discuss an embedding of certain full subcategory of $\operatorname{Mod}_{A}\left(\operatorname{Sp}_{k}\right)$ into the quasi-category of comodules associated to the adjunction.

\subsection{Quasi-categories of comodules}

In this subsection we shall introduce a quasi-category of comodules associated to an adjunction of quasi-categories.

First, we fix notation. For a monoidal quasi-category $\mathcal{M}$, we denote by $\operatorname{Alg}(\mathcal{M})$ the quasi-category of algebra objects in $\mathcal{M}$. For a quasi-category $\mathcal{N}$ left-tensored over $\mathcal{M}$ and an algebra object $T \in \operatorname{Alg}(\mathcal{M})$, we denote by $\operatorname{Mod}_{T}(\mathcal{N})$ the quasi-category of left $T$-modules in $\mathcal{N}$ (see [28, 4.1.1 and 4.1.2] for these concepts).

Let $\mathcal{C}$ and $\mathcal{D}$ be quasi-categories. We denote by $\operatorname{Fun}(\mathcal{C}, \mathcal{D})$ the quasi-category of functors from $\mathcal{C}$ to $\mathcal{D}$ (see $[27,1.2 .7]$ ). We can regard the quasi-category $\operatorname{End}(\mathcal{C})=$ $\operatorname{Fun}(\mathcal{C}, \mathcal{C})$ as a monoidal quasi-category by the composition of functors (see $[28,4.7]$ ). A monad on $\mathcal{C}$ is defined to be an algebra object of $\operatorname{End}(\mathcal{C})$. If $T$ is a monad on $\mathcal{C}$, we can consider the quasi-category of left $T$-modules $\operatorname{Mod}_{T}(\mathcal{C})$ in $\mathcal{C}$.

For quasi-categories $\mathcal{C}$ and $\mathcal{D}$, the quasi-category $\operatorname{Fun}(\mathcal{D}, \mathcal{C})$ carries a left action of the monoidal quasi-category $\operatorname{End}(\mathcal{C})$ by composition of functors, and we can regard $\operatorname{Fun}(\mathcal{D}, \mathcal{C})$ as left-tensored over $\operatorname{End}(\mathcal{C})$. Thus, we can consider the quasi-category of left $T$-modules $\operatorname{Mod}_{T}(\operatorname{Fun}(\mathcal{D}, \mathcal{C}))$ in $\operatorname{Fun}(\mathcal{D}, \mathcal{C})$ for a monad $T \in \operatorname{Alg}(\operatorname{End}(\mathcal{C}))$. Let $R: \mathcal{D} \rightarrow \mathcal{C}$ be a functor of quasi-categories. An endomorphism monad of $R$ consists of a monad $T \in \operatorname{Alg}(\operatorname{End}(\mathcal{C}))$ together with a left $T$-module $\bar{R} \in \operatorname{Mod}_{T}(\operatorname{Fun}(\mathcal{D}, \mathcal{C}))$ whose image in $\operatorname{Fun}(\mathcal{D}, \mathcal{C})$ coincides with $R$, such that the action map $a: T R \rightarrow R$ induces a weak equivalence of mapping spaces

$$
\operatorname{Map}_{\mathrm{Fun}(\mathcal{D}, \mathcal{C})}(F, R) \stackrel{T}{\longrightarrow} \operatorname{Map}_{\mathrm{Fun}(\mathcal{D}, \mathcal{C})}(T F, T R) \stackrel{a_{*}}{\longrightarrow} \operatorname{Map}_{\mathrm{Fun}(\mathcal{D}, \mathcal{C})}(T F, R)
$$

for any $F \in \operatorname{Fun}(\mathcal{D}, \mathcal{C})($ see $[28,4.7 .4])$.

Now we recall the definition of an adjunction between quasi-categories (see [27, Def. 5.2.2.1]). Let $\mathcal{C}$ and $\mathcal{D}$ be quasi-categories. An adjunction between $\mathcal{C}$ and $\mathcal{D}$ is a map $q: \mathcal{M} \rightarrow \Delta^{1}$ of simplicial sets which is both a Cartesian fibration and a coCartesian fibration together with equivalences $\mathcal{C} \rightarrow \mathcal{M}_{\{0\}}$ and $\mathcal{D} \rightarrow \mathcal{M}_{\{1\}}$, where $\mathcal{M}_{\{0\}}$ and $\mathcal{M}_{\{1\}}$ are the fibers of $q$ at $\{0\} \in \Delta^{1}$ and $\{1\} \in \Delta^{1}$, respectively (see [27, 2.4.2] for the definitions of a Cartesian fibration and a coCartesian fibration). In this case we let $L: \mathcal{C} \rightarrow \mathcal{D}$ and $R: \mathcal{D} \rightarrow \mathcal{C}$ be functors associated to $\mathcal{M}$, and say that $L$ is left adjoint to $R$ and $R$ is right adjoint to $L$.

We have a characterization of adjoint functors of quasi-categories in terms of mapping spaces as in classical category theory. Suppose we have a pair of functors of quasi-categories 
$L: \mathcal{C} \rightleftarrows \mathcal{D}: R$

The functor $L$ is left adjoint to $R$ if and only if there exists a morphism $u: \operatorname{id}_{\mathcal{C}} \rightarrow R L$ in $\operatorname{Fun}(\mathcal{C}, \mathcal{C})$ such that the composition

$$
\operatorname{Map}_{\mathcal{D}}(L(C), D) \stackrel{R}{\longrightarrow} \operatorname{Map}_{\mathcal{C}}(R L(C), R(D)) \stackrel{u_{C}^{*}}{\longrightarrow} \operatorname{Map}_{\mathcal{C}}(C, R(D))
$$

is a weak equivalence for any $C \in \mathcal{C}$ and $D \in \mathcal{D}$ (see [27, Prop. 5.2.2.8]).

We shall introduce a quasi-category of comodules associated to an adjunction of quasi-categories. For a quasi-category $\mathcal{X}$, we denote by $\mathcal{X}^{\text {op }}$ the opposite quasicategory of $\mathcal{X}$ (see $[27,1.2 .1]$ ). Suppose we have an adjunction of quasi-categories $L: \mathcal{C} \rightleftarrows \mathcal{D}: R$. This induces an adjunction of opposite quasi-categories

$$
R^{\mathrm{op}}: \mathcal{D}^{\mathrm{op}} \rightleftarrows \mathcal{C}^{\mathrm{op}}: L^{\mathrm{op}} \text {. }
$$

We obtain an object $L^{\mathrm{op}} R^{\mathrm{op}}$ in $\operatorname{End}\left(\mathcal{D}^{\mathrm{op}}\right)$, and we can lift $L^{\mathrm{op}} R^{\mathrm{op}}$ to an endomorphism monad of $L^{\text {op }}$ by [28, 4.7.4]. In particular, we have a monad $\Theta \in \operatorname{Alg}\left(\operatorname{End}\left(\mathcal{D}^{\text {op }}\right)\right)$ that is a lifting of $L^{\mathrm{op}} R^{\mathrm{op}}$, and a left $\Theta$-module object $\overline{L^{\mathrm{op}}} \in \operatorname{Mod}_{\Theta}\left(\operatorname{Fun}\left(\mathcal{C}^{\mathrm{op}}, \mathcal{D}^{\text {op }}\right)\right)$ that is a lifting of $L^{\mathrm{op}}$. We regard $\Theta$ as a comonad on $\mathcal{D}$ and we define the quasi-category of left $\Theta$-comodules

$$
\operatorname{Comod}_{\Theta}(\mathcal{D})
$$

to be $\operatorname{Mod}_{\Theta}\left(\mathcal{D}^{\mathrm{op}}\right)^{\mathrm{op}}$. Using an equivalence $\operatorname{Mod}_{\Theta}\left(\operatorname{Fun}\left(\mathcal{C}^{\mathrm{op}}, \mathcal{D}^{\mathrm{op}}\right)\right) \stackrel{\simeq}{\rightarrow}$ Fun $\left(\mathcal{C}^{\mathrm{op}}, \operatorname{Mod}_{\Theta}\left(\mathcal{D}^{\mathrm{op}}\right)\right)$, we obtain an object in $\operatorname{Fun}\left(\mathcal{C}^{\mathrm{op}}, \operatorname{Mod}_{\Theta}\left(\mathcal{D}^{\mathrm{op}}\right)\right)$ corresponding to $\overline{L^{\mathrm{op}}} \in \operatorname{Mod}_{\Theta}\left(\operatorname{Fun}\left(\mathcal{C}^{\mathrm{op}}, \mathcal{D}^{\mathrm{op}}\right)\right)$. Hence we see that the functor $L: \mathcal{C} \rightarrow \mathcal{D}$ factors through a functor

$$
\widetilde{L}: \mathcal{C} \longrightarrow \operatorname{Comod}_{\Theta}(\mathcal{D})
$$

so that $U \widetilde{L} \simeq L$, where $U: \operatorname{Comod}_{\Theta}(\mathcal{D}) \rightarrow \mathcal{D}$ is the forgetful functor. We say that $L$ exhibits $\mathcal{C}$ as comonadic over $\mathcal{D}$ if $\widetilde{L}$ is an equivalence of quasi-categories.

\subsection{Embeddings into quasi-categories of comodules}

Recall that $\mathrm{Sp}_{k}$ is the underlying quasi-category of the simplicial model category $\Sigma \mathrm{Sp}_{k}$. Since $\Sigma \mathrm{Sp}_{k}$ is a simplicial symmetric monoidal model category, $\mathrm{Sp}_{k}$ is a symmetric monoidal quasi-category (see [28, 4.1.3]). In this subsection we shall formulate embeddings of quasi-categories of modules into quasi-categories of comodules in $\mathrm{Sp}_{k}$.

For an algebra object $A$ in $\mathrm{Sp}_{k}$, we have the quasi-category of left $A$-modules $\operatorname{Mod}_{A}\left(\operatorname{Sp}_{k}\right)$ in $\operatorname{Sp}_{k}$. For a map $\psi: A \rightarrow E$ of algebra objects in $\operatorname{Sp}_{k}$, we have an adjunction of quasi-categories

$$
L: \operatorname{Mod}_{A}\left(\operatorname{Sp}_{k}\right) \rightleftarrows \operatorname{Mod}_{E}\left(\operatorname{Sp}_{k}\right): R
$$

where $L=E \wedge_{A}(-)$ and $R=\psi^{*}$. Hence we obtain a comonad $\Theta$ on $\operatorname{Mod}_{E}\left(\operatorname{Sp}_{k}\right)$ and a quasi-category of left $\Theta$-comodules 


$$
\operatorname{Comod}_{(E, \Theta)}\left(\operatorname{Sp}_{k}\right)=\operatorname{Comod}_{\Theta}\left(\operatorname{Mod}_{E}\left(\operatorname{Sp}_{k}\right)\right)
$$

The functor $L$ factors through a functor

$$
\text { Coex : } \operatorname{Mod}_{A}\left(\operatorname{Sp}_{k}\right) \longrightarrow \operatorname{Comod}_{(E, \Theta)}\left(\operatorname{Sp}_{k}\right)
$$

so that $U$ Coex $\simeq L$, where $U$ is the forgetful functor $U: \operatorname{Comod}_{(E, \Theta)}\left(\operatorname{Sp}_{k}\right) \rightarrow$ $\operatorname{Mod}_{E}\left(\operatorname{Sp}_{k}\right)$. We set

$$
\Theta^{\prime}=\operatorname{Coex} R \text {. }
$$

The functor $\Theta^{\prime}$ is informally given by $\Theta^{\prime}(X)=\Theta(X)$ with the obvious $\Theta$-comodule structure for $X \in \operatorname{Mod}_{E}\left(\operatorname{Sp}_{k}\right)$. Note that $\Theta^{\prime}$ is a right adjoint to the forgetful functor $U$.

Now we introduce a functor $P: \operatorname{Comod}_{(E, \Theta)}\left(\operatorname{Sp}_{k}\right) \rightarrow \operatorname{Mod}_{A}\left(\operatorname{Sp}_{k}\right)$ which is a derived functor of taking primitive elements. The functor $P$ is related to the derived completion defined by Carlsson in [7] and the nilpotent completion considered by Bousfield in [5]. For $X \in \operatorname{Comod}_{(E, \Theta)}\left(\operatorname{Sp}_{k}\right)$, we have a cosimplicial object

$$
C^{\bullet}(R, \Theta, U X)
$$

in $\operatorname{Mod}_{A}\left(\operatorname{Sp}_{k}\right)$ by the cobar construction. We define a functor

$$
P: \operatorname{Comod}_{(E, \Theta)}\left(\operatorname{Sp}_{k}\right) \rightarrow \operatorname{Mod}_{A}\left(\operatorname{Sp}_{k}\right)
$$

by $P X=\lim C^{\bullet}(R, \Theta, U X)$.

For $Y \in \operatorname{Mod}_{A}\left(\operatorname{Sp}_{k}\right)$, we have a coaugmented cosimplicial object

$$
Y \rightarrow E^{\bullet+1} Y
$$

in $\operatorname{Mod}_{A}\left(\operatorname{Sp}_{k}\right)$ given by

$$
E^{k+1} Y=\overbrace{E \wedge_{A} \cdots \wedge_{A} E}^{k+1} \wedge_{A} Y
$$

with the usual cosimplicial structure, which is sometimes called the Amitsur complex [2]. There is an equivalence of cosimplicial objects

$$
C^{\bullet}(R, \Theta, U \operatorname{Coex}(Y)) \simeq E^{\bullet+1} Y .
$$

Note that the map $Y \rightarrow \lim E^{\bullet+1} Y$ is an analogue of the derived completion of $Y$ at the $A$-algebra $E$ in the sense of [7] (see Remark 4.7 for the $k$-local Amitsur derived completion).

Furthermore, if $k=A=S$, then the map $Y \rightarrow \lim E^{\bullet+1} Y$ is the $E$-nilpotent completion of $Y$ in $\mathrm{Ho}(\mathrm{Sp})$ in the sense of [5], where $\mathrm{Ho}(\mathrm{Sp})$ is the stable homotopy category of spectra. 
We shall recall the $B$-nilpotent completion of spectra for a ring spectrum $B$ in $\mathrm{Ho}(\mathrm{Sp})$. A spectrum $W \in \mathrm{Ho}(\mathrm{Sp})$ is said to be $B$-nilpotent if $W$ lies in the thick ideal of $\mathrm{Ho}(\mathrm{Sp})$ generated by $B$. A $B$-nilpotent resolution of a spectrum $Z$ is a tower $\left\{W_{s}\right\}_{s \geq 0}$ under $Z$ in $\mathrm{Ho}(\mathrm{Sp})$ such that $W_{s}$ is $B$-nilpotent for all $s \geq 0$ and the map $\operatorname{colim}_{s} \operatorname{Hom}_{\mathrm{Ho}(\mathrm{Sp})}\left(W_{S}, N\right) \rightarrow \operatorname{Hom}_{\mathrm{Ho}(\mathrm{Sp})}(Z, N)$ is an isomorphism for any $B$-nilpotent spectrum $N$. The $B$-nilpotent completion of $Z$ is defined to be the homotopy inverse limit holim $W_{s}$ for any $B$-nilpotent resolution $\left\{W_{s}\right\}_{s \geq 0}$ of $Z$.

We can consider the Tot tower $\left\{\operatorname{Tor}^{s} E^{\bullet+1} Y\right\}_{s \geq 0}$ associated to the cosimplicial object $E^{\bullet+1} Y$. If $k=A=S$, then the cofiber of each map $\operatorname{Tot}^{s+1} E^{\bullet+1} Y \rightarrow \operatorname{Tot}^{s} E^{\bullet+1} Y$ is an $E$-module, and hence $E$-nilpotent in $\mathrm{Ho}(\mathrm{Sp})$ for all $s \geq 0$. By induction on $s$ and the fact that $\operatorname{Tot}^{0} E^{\bullet+1} Y=E \wedge Y$, we see that $\operatorname{Tot}^{s} E^{\bullet+1} Y$ is $E$-nilpotent for all $s \geq 0$. Furthermore, we let $F^{s}$ be the fiber of the map $Y \rightarrow \operatorname{Tot}^{s} E^{\bullet+1} Y$ for $s \geq 0$. The induced map $F^{s+1} \rightarrow F^{s}$ is null in $\mathrm{Ho}(\mathrm{Sp})$ after tensoring with $E$ for all $s \geq 0$. This implies an isomorphism $\operatorname{colim}_{s} \operatorname{Hom}_{\mathrm{Ho}(\mathrm{Sp})}\left(\operatorname{Tot}^{s} E^{\bullet+1} Y, N\right) \rightarrow \operatorname{Hom}_{\mathrm{Ho}(\mathrm{Sp})}(Y, N)$ for any $E$-nilpotent spectrum $N$. Hence $\lim E^{\bullet+1} Y$ is the $E$-nilpotent completion of $Y$ in $\mathrm{Ho}(\mathrm{Sp})$.

We would like to show that a certain full subcategory of $\operatorname{Mod}_{A}\left(\operatorname{Sp}_{k}\right)$ can be embedded into $\operatorname{Comod}_{(E, \Theta)}\left(\Sigma \mathrm{Sp}_{k}\right)$ through Coex. For this purpose, we show that Coex has a right adjoint.

Proposition 5.1 The functor $P$ is a right adjoint to Coex so that we have an adjunction of quasi-categories

$$
\text { Coex : } \operatorname{Mod}_{A}\left(\operatorname{Sp}_{k}\right) \rightleftarrows \operatorname{Comod}_{(E, \Theta)}\left(\operatorname{Sp}_{k}\right): P \text {. }
$$

Proof Let $\mathcal{X}$ be the full subcategory of $\operatorname{Comod}_{(E, \Theta)}\left(\operatorname{Sp}_{k}\right)$ consisting of $X$ such that the functor

$$
\operatorname{Map}_{\operatorname{Comod}_{(E, \Theta)}\left(\operatorname{Sp}_{k}\right)}(\operatorname{Coex}(-), X): \operatorname{Mod}_{A}\left(\operatorname{Sp}_{k}\right) \rightarrow \mathcal{S}
$$

is representable, where $\mathcal{S}$ is the quasi-category of spaces. We denote by $\widetilde{P}(X)$ the representing object in $\operatorname{Mod}_{A}\left(\operatorname{Sp}_{k}\right)$ for $X \in \mathcal{X}$. In this case, $\widetilde{P}(X)$ is well-defined up to canonical equivalence and we obtain a functor

$$
\widetilde{P}: \mathcal{X} \rightarrow \operatorname{Mod}_{A}\left(\operatorname{Sp}_{k}\right)
$$

In order to prove the proposition, we have to show that $\mathcal{X}$ is actually the whole quasi-category $\operatorname{Comod}_{(E, \Theta)}\left(\operatorname{Sp}_{k}\right)$. First, we shall show that $\Theta^{\prime} Z \in \mathcal{X}$ for any $Z \in$ $\operatorname{Mod}_{E}\left(\operatorname{Sp}_{k}\right)$. Since $\Theta^{\prime}$ is a right adjoint to $U$ and $U$ Coex $\simeq L$ is a left adjoint to $R$, we see that there is a natural equivalence

$$
\operatorname{Map}_{\operatorname{Comod}_{(E, \Theta)}\left(\operatorname{Sp}_{k}\right)}\left(\operatorname{Coex}(Y), \Theta^{\prime} Z\right) \simeq \operatorname{Map}_{\operatorname{Mod}_{A}\left(\operatorname{Sp}_{k}\right)}(Y, R Z)
$$

for any $Y \in \operatorname{Mod}_{E}\left(\operatorname{Sp}_{k}\right)$, and hence $\Theta^{\prime} Z \in \mathcal{X}$. We note that $\widetilde{P} \Theta^{\prime} Z \simeq R Z$. 
Next, we shall show that $X \in \mathcal{X}$ for any $X \in \operatorname{Comod}_{(E, \Theta)}\left(\operatorname{Sp}_{k}\right)$. Let $C^{\bullet}\left(\Theta^{\prime}, \Theta, U X\right)$ be a cosimplicial object in $\operatorname{Comod}_{(E, \Theta)}\left(\operatorname{Sp}_{k}\right)$ given by the cobar construction. By [28, 4.7.4], we see that $\lim C^{\bullet}\left(\Theta^{\prime}, \Theta, U X\right) \simeq X$. Since $C^{n}\left(\Theta^{\prime}, \Theta, U X\right) \simeq$ $\Theta^{\prime} \Theta^{n} U X \in \mathcal{X}$ for any $n$, we can construct a cosimplicial object $\widetilde{P} C^{\bullet}\left(\Theta^{\prime}, \Theta, U X\right)$ in $\operatorname{Mod}_{A}\left(\operatorname{Sp}_{k}\right)$. We can verify that $\lim \widetilde{P} C^{\bullet}\left(\Theta^{\prime}, \Theta, U X\right)$ represents the functor $\operatorname{Map}_{\operatorname{Comod}}^{(E, \Theta)}\left(\operatorname{Sp}_{k}\right)(\operatorname{Coex}(-), X)$, and hence $X \in \mathcal{X}$.

Therefore, we obtain $\mathcal{X}=\operatorname{Comod}_{(E, \Theta)}\left(\operatorname{Sp}_{k}\right)$. Since there is an equivalence $\widetilde{P} C^{\bullet}\left(\Theta^{\prime}, \Theta, U X\right) \simeq C^{\bullet}(R, \Theta, U X)$ of cosimplicial objects, we see that $\widetilde{P} X \simeq P X$ for any $X$. This completes the proof.

Let $\mathcal{T}$ be the full subcategory of $\operatorname{Mod}_{A}\left(\mathrm{Sp}_{k}\right)$ consisting of $X$ such that the unit map $X \rightarrow P \operatorname{Coex}(X)$ is an equivalence

$$
\mathcal{T}=\left\{X \in \operatorname{Mod}_{A}\left(\operatorname{Sp}_{k}\right) \mid X \stackrel{\simeq}{\rightarrow} P \operatorname{Coex}(X)\right\}
$$

In the same way as in Proposition 4.5, we obtain the following proposition.

Proposition 5.2 The restriction of Coex to $\mathcal{T}$ is a fully faithful functor of quasicategories.

\subsection{Examples}

In this subsection we give some examples of embeddings into quasi-categories of comodules.

1. (cf. [17, 6.1.2]) Let $k=S$ and let $\psi: S \rightarrow M U$ be the unit map, where $M U$ is the complex cobordism spectrum. We have an adjunction $M U \wedge(-): \mathrm{Sp} \rightleftarrows$ $\operatorname{Mod}_{M U}(\mathrm{Sp}): \psi^{*}$. We denote by $M U \wedge M U$ the comonad on $\operatorname{Mod}_{M U}(\mathrm{Sp})$ associated to the adjoint pair $\left(M U \wedge(-), \psi^{*}\right)$. If $X \in \mathrm{Sp}$ is connective, then the map $X \rightarrow \lim M U^{\bullet+1} X$ is an equivalence by [5, Thm. 6.5]. Hence $X \in \mathcal{T}$. Let $\mathrm{Sp}^{\geq 0}$ be the full subcategory of Sp consisting of connective spectra. By Proposition 5.2, the functor

$$
M U \wedge(-): \mathrm{Sp}^{\geq 0} \longrightarrow \operatorname{Comod}_{(M U, M U \wedge M U)}(\mathrm{Sp})
$$

is fully faithful.

2. Let $k=E(n)$ be the $n$th Johnson-Wilson spectrum at a prime $p$ and let $\psi: S \rightarrow E(n)$ be the unit map. We have an adjunction $E(n) \wedge(-):$ $\operatorname{Sp}_{E(n)} \rightleftarrows \operatorname{Mod}_{E(n)}\left(\operatorname{Sp}_{E(n)}\right): \psi^{*}$. We denote by $E(n) \wedge E(n)$ the comonad on $\operatorname{Mod}_{E(n)}\left(\operatorname{Sp}_{E(n)}\right)$ associated to the adjoint pair $\left(E(n) \wedge(-), \psi^{*}\right)$. For any $X \in \operatorname{Sp}_{E(n)}$, the map $X \rightarrow \lim E(n)^{\bullet+1} X$ is an equivalence in $\operatorname{Sp}_{E(n)}$ since any spectrum is $E(n)$-prenilpotent by [22, Thm. 5.3]. Hence the functor

$$
E(n) \wedge(-): \operatorname{Sp}_{E(n)} \longrightarrow \operatorname{Comod}_{(E(n), E(n) \wedge E(n))}\left(\operatorname{Sp}_{E(n)}\right)
$$

is fully faithful. 
3. Let $k=K(n)$ and let $\psi: S \rightarrow E_{n}$ be the unit map. We have an adjunction $L_{K(n)}\left(E_{n} \wedge(-)\right): \operatorname{Sp}_{K(n)} \rightleftarrows \operatorname{Mod}_{E_{n}}\left(\operatorname{Sp}_{K(n)}\right): \psi^{*}$. We denote by $L_{K(n)}\left(E_{n} \wedge E_{n}\right)$ the comonad on $\operatorname{Mod}_{E_{n}}\left(\operatorname{Sp}_{K(n)}\right)$ associated to the adjoint pair $\left(L_{K(n)}\left(E_{n} \wedge(-)\right), \psi^{*}\right)$. For any $X \in \operatorname{Sp}_{K(n)}$, the map $X \rightarrow \lim L_{K(n)} E_{n}^{\bullet+1} X$ is an equivalence in $\operatorname{Sp}_{K(n)}$ since any $K(n)$-local spectrum is $K(n)$-local $E_{n}$-nilpotent by [11, Prop. A.3]. Hence the functor

$$
L_{K(n)}\left(E_{n} \wedge(-)\right): \operatorname{Sp}_{K(n)} \longrightarrow \operatorname{Comod}_{\left(E_{n}, L_{K(n)}\left(E_{n} \wedge E_{n}\right)\right)}\left(\operatorname{Sp}_{K(n)}\right)
$$

is fully faithful.

4. Let $k=M(p)$ be the $\bmod p$ Moore spectrum at a prime $p$. Let $\psi: S \rightarrow H \mathbb{F}_{p}$ be the unit map, where $H \mathbb{F}_{p}$ is the mod $p$ Eilenberg-Mac Lane spectrum. We have an adjunction $H \mathbb{F}_{p} \wedge(-): \operatorname{Sp}_{M(p)} \rightleftarrows \operatorname{Mod}_{H \mathbb{F}_{p}}\left(\operatorname{Sp}_{M(p)}\right): \psi^{*}$. We denote by $H \mathbb{F}_{p} \wedge H \mathbb{F}_{p}$ the comonad on $\operatorname{Mod}_{H \mathbb{F}_{p}}\left(\operatorname{Sp}_{M(p)}\right)$ associated to the adjoint pair $\left(H \mathbb{F}_{p} \wedge(-), \psi^{*}\right)$. If $X \in \operatorname{Sp}_{M(p)}$ is connective, then the map $X \rightarrow \lim M \mathbb{F}_{p}^{\bullet+1} X$ is an equivalence in $\operatorname{Sp}_{M(p)}$ by [5, Thm. 6.5], and hence $X \in \mathcal{T}$. The functor

$$
H \mathbb{F}_{p} \wedge(-): \operatorname{Sp}_{M(p)}^{\geq 0} \longrightarrow \operatorname{Comod}_{\left(H \mathbb{F}_{p}, H \mathbb{F}_{p} \wedge H \mathbb{F}_{p}\right)}\left(\operatorname{Sp}_{M(p)}\right)
$$

is fully faithful, where $\operatorname{Sp}_{M(p)}^{\geq 0}$ is the full subcategory of $\operatorname{Sp}_{M(p)}$ consisting of connective spectra.

\section{Quasi-category of discrete $G$-spectra}

In this section we discuss the underlying quasi-categories of modules and algebras over $\Sigma \operatorname{Sp}(G)_{k}$. We show that the formulations of embeddings of module categories in Sects. 4 and 5 are equivalent under some conditions.

\subsection{The quasi-category $\operatorname{Sp}(G)_{k}$ as a comodule category}

By Proposition 3.10, we have a symmetric monoidal $\Sigma$ Sp-Quillen adjunction $U$ : $\Sigma \operatorname{Sp}(G)_{k} \rightleftarrows \Sigma \operatorname{Sp}_{k}: V$, where $U$ is the forgetful functor and $V(-)=\operatorname{Map}_{c}(G,-)$. We denote by $\operatorname{Sp}(G)_{k}$ the underlying quasi-category of $\Sigma \operatorname{Sp}(G)_{k}$. The adjoint pair $(U, V)$ induces an adjunction of quasi-categories

$$
U_{k}: \operatorname{Sp}(G)_{k} \rightleftarrows \operatorname{Sp}_{k}: V_{k}
$$

We denote by $\Gamma$ the comonad on $\mathrm{Sp}_{k}$ associated to the adjoint pair $\left(U_{k}, V_{k}\right)$. We have the quasi-category of comodules

\section{$\operatorname{Comod}_{\Gamma}\left(\operatorname{Sp}_{k}\right)$}

over $\Gamma$. In this subsection we shall show that $U_{k}$ exhibits $\operatorname{Sp}(G)_{k}$ as comonadic over $\mathrm{Sp}_{k}$, that is, $\operatorname{Sp}(G)_{k}$ is equivalent to $\operatorname{Comod}_{\Gamma}\left(\operatorname{Sp}_{k}\right)$ under some conditions. 
First, we consider the unlocalized version of the adjunction $U: \operatorname{Sp}(G) \rightleftarrows \operatorname{Sp}: V$ and show that the forgetful functor $U: \operatorname{Sp}(G) \rightarrow \operatorname{Sp}$ exhibits $\operatorname{Sp}(G)$ as comonadic over Sp. For this purpose, we shall apply the quasi-categorical Barr-Beck theorem by Lurie $[28,4.7 .4]$. In particular, we have to show that $U$ preserves the limit of any cosimplicial object of $\Sigma \operatorname{Sp}(G)$ that is split in $\Sigma$ Sp. Since the limit of a diagram in the underlying quasi-category of a simplicial model category is represented by the homotopy limit of the simplicial model category, we recall the homotopy limit of a diagram in a simplicial model category.

We use a model of homotopy limits in [18, Ch. 18]. For a small category $\mathcal{C}$ and an object $\alpha \in \mathcal{C}$, we denote by $(\mathcal{C} \downarrow \alpha)$ the category of objects of $\mathcal{C}$ over $\alpha$. An object of $(\mathcal{C} \downarrow \alpha)$ is a pair $(\beta, \sigma)$ where $\beta$ is an object of $\mathcal{C}$ and $\sigma$ is a map $\beta \rightarrow \alpha$ in $\mathcal{C}$. A morphism from $(\beta, \sigma)$ to $\left(\beta^{\prime}, \sigma^{\prime}\right)$ in $(\mathcal{C} \downarrow \alpha)$ is a map $\tau: \beta \rightarrow \beta^{\prime}$ in $\mathcal{C}$ such that $\sigma=\sigma^{\prime} \tau$. A map $\sigma: \alpha \rightarrow \alpha^{\prime}$ in $\mathcal{C}$ induces a functor $\sigma_{*}:(\mathcal{C} \downarrow \alpha) \rightarrow\left(\mathcal{C} \downarrow \alpha^{\prime}\right)$ by composing with $\sigma$. For a small category $\mathcal{C}$, we denote by $B \mathcal{C}$ its classifying space (simplicial set), which is given by applying the nerve functor to $\mathcal{C}$. Let $\mathbf{M}$ be a simplicial model category. For an object $\mathbf{Y} \in \mathbf{M}$ and a simplicial set $K$, we denote by $\mathbf{Y}^{K}$ the power of $\mathbf{Y}$ by $K$. For a functor $\mathbf{X}: \mathcal{C} \rightarrow \mathbf{M}$ such that $\mathbf{X}_{\alpha}$ is fibrant for all $\alpha \in \mathcal{C}$, the homotopy limit holim $\mathcal{C}_{\mathcal{C}}^{\mathbf{M}} \mathbf{X}$ is defined to be the equalizer of the maps

$$
\prod_{\alpha \in \mathcal{C}}\left(\mathbf{X}_{\alpha}\right)^{B(\mathcal{C} \downarrow \alpha)} \underset{\psi}{\stackrel{\phi}{\rightrightarrows}} \prod_{\left(\sigma: \alpha \rightarrow \alpha^{\prime}\right) \in \mathcal{C}}\left(\mathbf{X}_{\alpha^{\prime}}\right)^{B(\mathcal{C} \downarrow \alpha)},
$$

where the projection of the map $\phi$ on the factor $\sigma: \alpha \rightarrow \alpha^{\prime}$ is the composition of the projection on the factor $\alpha$ with the map $\left(\mathbf{X}_{\alpha}\right)^{B(\mathcal{C} \downarrow \alpha)} \rightarrow\left(\mathbf{X}_{\alpha^{\prime}}\right)^{B(\mathcal{C} \downarrow \alpha)}$ induced by the map $\mathbf{X}_{\sigma}: \mathbf{X}_{\alpha} \rightarrow \mathbf{X}_{\alpha^{\prime}}$ and the projection of the map $\psi$ on the factor $\sigma: \alpha \rightarrow \alpha^{\prime}$ is the composition of the projection on the factor $\alpha^{\prime}$ with the map $\left(\mathbf{X}_{\alpha^{\prime}}\right)^{B\left(\mathcal{C} \downarrow \alpha^{\prime}\right)} \rightarrow$ $\left(\mathbf{X}_{\alpha^{\prime}}\right)^{B(\mathcal{C} \downarrow \alpha)}$ induced by the map $B\left(\sigma_{*}\right): B(\mathcal{C} \downarrow \alpha) \rightarrow B\left(\mathcal{C} \downarrow \alpha^{\prime}\right)$.

Next, we would like to compare the homotopy limit of a diagram in $\Sigma \operatorname{Sp}(G)$ with that of the diagram in $\Sigma$ Sp obtained by applying the forgetful functor $U$. For this purpose, we shall describe the limits and powers in $\Sigma \operatorname{Sp}(G)$ in terms of those in $\Sigma$ Sp.

For a small category $\mathcal{C}$, we let $\mathbf{X}: \mathcal{C} \rightarrow \Sigma \operatorname{Sp}(G)$ be a functor. We shall describe the limit $\lim _{\mathcal{C}}^{\Sigma \operatorname{Sp}(G)} \mathbf{X}$ in $\Sigma \operatorname{Sp}(G)$. We have the induced functor $U \mathbf{X}: \mathcal{C} \rightarrow \Sigma$ Sp and the limit $\lim _{\mathcal{C}}^{\Sigma S p} U \mathbf{X}$ in $\Sigma$ Sp. By the functoriality of limit, we can regard $\lim _{\mathcal{C}}^{\Sigma S p} U \mathbf{X}$ as an object in $\Sigma \operatorname{Sp}\left(G^{\delta}\right)$. We also have the induced functor $\mathbf{X}^{\delta}: \mathcal{C} \rightarrow \Sigma \operatorname{Sp}\left(G^{\delta}\right)$. We see that $\lim _{\mathcal{C}}^{\Sigma S p} U \mathbf{X}$ can be identified with the limit of $\mathbf{X}^{\delta}$ in $\Sigma \operatorname{Sp}\left(G^{\delta}\right): \lim _{\mathcal{C}}^{\Sigma \operatorname{Sp}\left(G^{\delta}\right)} \mathbf{X}^{\delta} \cong$ $\lim _{\mathcal{C}}^{\Sigma \operatorname{Sp}} U \mathbf{X}$. Since $d: \Sigma \operatorname{Sp}\left(G^{\delta}\right) \rightarrow \Sigma \operatorname{Sp}(G)$ is right adjoint, the functor $d$ preserves limits, and hence we obtain that the $\operatorname{limit}_{\lim _{\mathcal{C}}}^{\Sigma \operatorname{Sp}(G)} \mathbf{X}$ in $\Sigma \operatorname{Sp}(G)$ is isomorphic to $d\left(\lim _{\mathcal{C}}^{\Sigma \operatorname{Sp}\left(G^{\delta}\right)} \mathbf{X}^{\delta}\right)$ and hence we obtain an isomorphism

$$
\lim _{\mathcal{C}}^{\Sigma S p(G)} \mathbf{X} \cong d\left(\lim _{\mathcal{C}}^{\Sigma S p} U \mathbf{X}\right)
$$

Let $X \in \Sigma \operatorname{Sp}(G)$ be a discrete symmetric $G$-spectrum and $K$ a simplicial set. We shall describe the power $X^{K}$ of $X$ by $K$ in $\Sigma \operatorname{Sp}(G)$. Note that the copower $X \otimes K$ is 
given by $X \wedge \Sigma_{+}^{\infty} K$, where $\Sigma_{+}^{\infty} K$ is the suspension spectrum of $K$ with the disjoint base point. This implies that the functor $\delta$ preserves copower, that is, there is a natural isomorphism $(X \otimes K)^{\delta} \cong X^{\delta} \otimes K$ in $\Sigma \operatorname{Sp}\left(G^{\delta}\right)$ for any $X \in \Sigma \operatorname{Sp}(G)$ and any simplicial set $K$. By using the adjunction of power and copower, we see that the functor $d$ preserves powers, that is, $d\left(Y^{K}\right) \cong(d Y)^{K}$ for any $Y \in \Sigma \operatorname{Sp}\left(G^{\delta}\right)$ and any simplicial set $K$. By the functoriality of power, we can regard the power $(U X)^{K}$ of the symmetric spectrum $U X \in \Sigma \operatorname{Sp}$ by $K$ as an object of $\Sigma \operatorname{Sp}\left(G^{\delta}\right)$ and identify the power $\left(X^{\delta}\right)^{K}$ in $\Sigma \operatorname{Sp}\left(G^{\delta}\right)$ with $(U X)^{K}$. Using $X \cong d\left(X^{\delta}\right)$, we obtain an isomorphism

$$
X^{K} \cong d\left((U X)^{K}\right)
$$

in $\Sigma \operatorname{Sp}(G)$ for any $X \in \Sigma \operatorname{Sp}(G)$ and any simplicial set $K$.

Using these descriptions of limits and powers in $\Sigma \operatorname{Sp}(G)$, we shall describe the homotopy limit of a diagram in $\Sigma \operatorname{Sp}(G)$. Let $\mathcal{C}$ be a small category and let $\mathbf{X}: \mathcal{C} \rightarrow$ $\Sigma \operatorname{Sp}(G)$ be a functor such that $\mathbf{X}_{\alpha}$ is fibrant for all $\alpha \in \mathcal{C}$. By the functoriality of the homotopy limit, we can regard the homotopy $\operatorname{limit}_{\operatorname{holim}}{ }_{\mathcal{C}}^{\Sigma \mathrm{Sp}} U \mathbf{X}$ in $\Sigma$ Sp of the induced functor $U \mathbf{X}$ as an object of $\Sigma \operatorname{Sp}\left(G^{\delta}\right)$. Note that $U \mathbf{X}_{\alpha}$ is fibrant for all $\alpha$ since $U$ preserves fibrant objects by [4, Cor. 5.3.3]. Since $d$ preserves limits and powers, we obtain an isomorphism

$$
\operatorname{holim}_{\mathcal{C}}^{\Sigma S p(G)} \mathbf{X} \cong d\left(\operatorname{holim}_{\mathcal{C}}^{\Sigma S p} U \mathbf{X}\right)
$$

We use the following notation for simplicity. For a cosimplicial object $Y^{\bullet}$ in $\Sigma$ Sp such that $Y^{r}$ is fibrant for all $r \geq 0$, we denote by

$$
\operatorname{holim}_{\Delta} Y^{\bullet}
$$

the homotopy $\operatorname{limit}_{\text {holim }}{ }_{\Delta}^{\Sigma \mathrm{Sp}} Y^{\bullet}$ in $\Sigma \mathrm{Sp}$, and for a cosimplicial object $Z^{\bullet}$ in $\Sigma \operatorname{Sp}(G)$ such that $Z^{r}$ is fibrant for all $r \geq 0$, we denote by

$$
\operatorname{holim}_{\Delta}^{G} Z^{\bullet}
$$

the homotopy limit of $Z^{\bullet}$ in $\Sigma \operatorname{Sp}(G)$. By the above argument, we have an isomorphism

$$
\operatorname{holim}_{\Delta}^{G} Z^{\bullet} \cong d\left(\operatorname{holim}_{\Delta} U Z^{\bullet}\right)
$$

for any cosimplicial object $Z^{\bullet}$ in $\Sigma \operatorname{Sp}(G)$ such that $Z^{r}$ is fibrant for all $r \geq 0$.

We have a canonical map

$$
U \operatorname{holim}_{\Delta}^{G} Z^{\bullet} \longrightarrow \operatorname{holim}_{\Delta} U Z^{\bullet}
$$

in $\Sigma$ Sp. We give a sufficient condition to ensure that the canonical map is an equivalence. The following lemma will be used to show that the forgetful functor $U$ preserves the limit of any $U$-split cosimplicial object of $\operatorname{Sp}(G)$. 
Lemma 6.1 Let $Z^{\bullet}$ be a cosimplicial object in $\Sigma \operatorname{Sp}(G)$ such that $Z^{r}$ is fibrant for all $r \geq 0$. We set $Z=\operatorname{holim}_{\Delta}^{G} Z^{\bullet}$ and $W=\operatorname{holim}_{\Delta} U Z^{\bullet}$. If $G$ has finite virtual cohomological dimension, the coaugmentation map $W \rightarrow U Z^{\bullet}$ induces an isomorphism $\pi_{*}(W) \stackrel{\cong}{\rightarrow} \pi^{0} \pi_{*}\left(U Z^{\bullet}\right)$, and $\pi^{s} \pi_{*}\left(U Z^{\bullet}\right)=0$ for all $s>0$, then the canonical map $U Z \rightarrow W$ is a weak equivalence.

Proof Since $G$ has finite virtual cohomological dimension, as in the second paragraph of the proof of [9, Thm. 7.4], we can choose a positive integer $m$ and a fundamental neighborhood system $\mathcal{N}$ of the identity element of $G$ consisting of open normal subgroups $N$ such that the cohomological dimension $\operatorname{cd}(N) \leq m$. In the following of this proof, we fix such a system $\mathcal{N}$. Since $\mathcal{N}$ is a fundamental neighborhood system of the identity, we have $d(X) \cong \operatorname{colim}_{N \in \mathcal{N}} X^{N}$ for any $X \in \Sigma \operatorname{Sp}(G)$. Hence we obtain an isomorphism

$$
\begin{aligned}
\operatorname{holim}_{\Delta}^{G} Z^{\bullet} & \cong d\left(\operatorname{holim}_{\Delta} U Z^{\bullet}\right) \\
& \cong \operatorname{colim}_{N \in \mathcal{N}} \operatorname{holim}_{\Delta}\left(Z^{\bullet}\right)^{N} .
\end{aligned}
$$

Since the discrete $G$-spectrum $Z^{r}$ is fibrant in $\Sigma \operatorname{Sp}(G)$, by [4, Prop. 3.3.1(2)], $Z^{r}$ is fibrant in $\Sigma \operatorname{Sp}(N)$ for any $r \geq 0$ and any $N \in \mathcal{N}$. Hence we see that the fixed points spectrum $\left(Z^{r}\right)^{N}$ is equivalent to the homotopy fixed points spectrum $\left(Z^{r}\right)^{h N}$ for any $r \geq 0$ and any $N \in \mathcal{N}$. Since $N \in \mathcal{N}$ has finite cohomological dimension, by [9, Thm. 7.4], we have $\left(Z^{r}\right)^{N} \simeq \operatorname{holim}_{\Delta} \operatorname{Map}_{c}\left(G^{\bullet+1}, Z^{r}\right)^{N}$. Hence we obtain

$$
\operatorname{holim}_{\Delta}^{G} Z^{\bullet} \simeq \operatorname{colim}_{N \in \mathcal{N}} \operatorname{holim}_{\Delta} \operatorname{holim}_{\Delta} \operatorname{Map}_{c}\left(G^{\bullet+1}, Z^{\bullet}\right)^{N}
$$

We fix $N \in \mathcal{N}$ and $k \geq 0$, and consider the cosimplicial spectrum $\operatorname{Map}_{c}\left(G^{k+1}, Z^{\bullet}\right)^{N}$. The Bousfield-Kan spectral sequence abutting to the homotopy groups of $\operatorname{holim}_{\Delta} \operatorname{Map}_{c}\left(G^{k+1}, Z^{\bullet}\right)^{N}$ has the form

$$
{ }_{I} E_{2}^{s, t} \cong \pi^{s} \pi_{t} \operatorname{Map}_{c}\left(G^{k+1}, Z^{\bullet}\right)^{N} \Longrightarrow \pi_{t-s} \operatorname{holim}_{\Delta} \operatorname{Map}_{c}\left(G^{k+1}, Z^{\bullet}\right)^{N} .
$$

Since $\pi_{*} \operatorname{Map}_{c}\left(G^{k+1}, Z^{r}\right)^{N} \cong \operatorname{Map}_{c}\left(G^{k+1}, \pi_{*}\left(Z^{r}\right)\right)^{N}$ for any $r \geq 0$, we see that ${ }_{I} E_{2}^{s, t}=0$ for $s>0$ and ${ }_{I} E_{2}^{0, t} \cong \operatorname{Map}_{c}\left(G^{k+1}, \pi_{t}(W)\right)^{N}$ by the assumptions on the cohomotopy groups of $\pi_{*}\left(U Z^{\bullet}\right)$ and the fact that $\operatorname{Map}_{c}\left(G^{k+1},-\right)^{N}$ is an exact functor from the category of discrete $G$-modules to the category of abelian groups. Note that $\pi_{t}(W)$ is a discrete $G$-module since it is a submodule of the discrete $G$ module $\pi_{t}\left(Z^{0}\right)$. Hence the spectral sequence collapses from the $E_{2}$-page and we obtain an isomorphism

$$
\pi_{*} \operatorname{holim}_{\Delta} \operatorname{Map}_{c}\left(G^{k+1}, Z^{\bullet}\right)^{N} \cong \operatorname{Map}_{c}\left(G^{k+1}, \pi_{*}(W)\right)^{N} .
$$

Now we consider the cosimplicial spectrum $\operatorname{holim}_{[r] \in \Delta} \operatorname{Map}_{c}\left(G^{\bullet+1}, Z^{r}\right)^{N}$. The Bousfield-Kan spectral sequence abutting to the homotopy groups of $\operatorname{holim}_{\Delta} \operatorname{holim}_{[r] \in \Delta} \operatorname{Map}_{c}\left(G^{\bullet+1}, Z^{r}\right)^{N}$ has the form

$$
{ }_{I I} E_{2}^{s, t} \cong \pi^{s} \pi_{t} \operatorname{holim}_{[r] \in \Delta} \operatorname{Map}_{c}\left(G^{\bullet+1}, Z^{r}\right)^{N}
$$




$$
\Longrightarrow \pi_{t-s} \operatorname{holim}_{\Delta} \operatorname{holim}_{[r] \in \Delta} \operatorname{Map}_{c}\left(G^{\bullet+1}, Z^{r}\right)^{N}
$$

Since $\pi_{t} \operatorname{holim}_{[r] \in \Delta} \operatorname{Map}_{c}\left(G^{\bullet+1}, Z^{r}\right)^{N} \cong \operatorname{Map}_{c}\left(G^{\bullet+1}, \pi_{t}(W)\right)^{N}$, we see that ${ }_{I I} E_{2}^{s, t} \cong$ $H_{c}^{s}\left(N ; \pi_{t}(W)\right)$.

Since the cohomological dimension $\operatorname{cd}(N)$ is uniformly bounded for $N \in \mathcal{N}$, by taking the colimit over $N \in \mathcal{N}$ of the spectral sequences (6.1), we obtain a spectral sequence

$$
{ }_{I I I} E_{2}^{p, q} \cong \operatorname{colim}_{N \in \mathcal{N}} H_{c}^{p}\left(N ; \pi_{q}(W)\right) \Longrightarrow \pi_{q-p}(Z)
$$

as in the proof of [9, Thm. 7.4]. Since ${ }_{I I I} E_{2}^{0, q} \cong \pi_{q}(W)$ and ${ }_{I I I} E_{2}^{p, q}=0$ for $p>0$, we see that the inclusion map $U Z \rightarrow W$ is a weak equivalence in $\Sigma$ Sp.

Let $F: \mathcal{C} \rightarrow \mathcal{D}$ be a functor of quasi-categories and $X^{\bullet}$ a cosimplicial object of $\mathcal{C}$. We say that $X^{\bullet}$ is $F$-split if $F X^{\bullet}$ is a split cosimplicial object of $\mathcal{D}$ (see $[28,4.7 .3]$ for the definition of a split simplicial object).

We consider the forgetful functor $U: \operatorname{Sp}(G) \rightarrow \mathrm{Sp}$ and a $U$-split cosimplicial object of $\operatorname{Sp}(G)$. Note that $\operatorname{Sp}(G)$ admits all small limits by [27, Cor. 4.2.4.8] since $\operatorname{Sp}(G)$ is the underlying quasi-category of the combinatorial simplicial model category $\Sigma \operatorname{Sp}(G)$. In particular, the limit of any cosimplicial object exists. We obtain the following lemma by Lemma 6.1.

Lemma 6.2 If $G$ has finite virtual cohomological dimension, then the forgetful functor $U: \operatorname{Sp}(G) \rightarrow \operatorname{Sp}$ preserves the limit of any $U$-split cosimplicial object of $\operatorname{Sp}(G)$.

Proof We recall that $\mathbf{M}^{\circ}$ is the full simplicial subcategory of a simplicial model category $\mathbf{M}$ consisting of objects that are both fibrant and cofibrant, and that $N\left(\mathbf{M}^{\circ}\right)$ is the underlying quasi-category of $\mathbf{M}$, where $N(-)$ is the simplicial nerve functor.

Let $X^{\bullet}$ be a cosimplicial object in $\operatorname{Sp}(G)$ that is $U$-split. By [27, Prop. 4.2.4.4], there is a cosimplicial object $Y^{\bullet}$ in $\Sigma \operatorname{Sp}(G)^{\circ}$ such that $N\left(Y^{\bullet}\right) \simeq X^{\bullet}$. Note that $U Y^{\bullet}$ is a cosimplicial object in $\Sigma \mathrm{Sp}^{\circ}$ since $U$ is a left Quillen functor and preserves fibrant objects by [4, Cor. 5.3.3]. In order to prove the lemma, we have to show that the map

$$
\varphi: U\left(\operatorname{holim}_{\Delta}^{G} Y^{\bullet}\right) \longrightarrow \operatorname{holim}_{\Delta} U Y^{\bullet}
$$

is a weak equivalence.

Since $U X^{\bullet}$ is a split cosimplicial object in $\operatorname{Sp}, \pi_{*}\left(U X^{\bullet}\right)=\pi_{*}\left(U Y^{\bullet}\right)$ is a split cosimplicial object in the category of graded modules. In particular, the coaugmentation map induces an isomorphism $\pi_{*}\left(\operatorname{holim}_{\Delta} U Y^{\bullet}\right) \stackrel{\cong}{\rightarrow} \pi^{0} \pi_{*}\left(U Y^{\bullet}\right)$, and $\pi^{s} \pi_{*}\left(U Y^{\bullet}\right)=0$ for all $s>0$. By Lemma 6.1, we see that the map $\varphi$ is a weak equivalence. This completes the proof.

Using Lemma 6.2, we obtain the following proposition which shows that $\operatorname{Sp}(G)$ is comonadic over Sp. 
Proposition 6.3 If $G$ has finite virtual cohomological dimension, then the forgetful functor $U: \operatorname{Sp}(G) \rightarrow \operatorname{Sp}$ exhibits $\operatorname{Sp}(G)$ as comonadic over $\mathrm{Sp}$, that is, we have an equivalence of quasi-categories

$$
\operatorname{Sp}(G) \stackrel{\simeq}{\longrightarrow} \operatorname{Comod}_{\Gamma}(\mathrm{Sp})
$$

Proof We shall use the quasi-categorical Barr-Beck theorem by Lurie [28, 4.7.4]. We have to show that $U$ is conservative, $\operatorname{Sp}(G)$ admits a limit for any $U$-split cosimplicial object, and the limit of any $U$-split cosimplicial object is preserved by $U$.

By the definition of the weak equivalences in $\Sigma \operatorname{Sp}(G)$, we see that the forgetful functor $U: \operatorname{Sp}(G) \rightarrow$ Sp is conservative. Since $\operatorname{Sp}(G)$ is the underlying quasi-category of the simplicial model category $\Sigma \operatorname{Sp}(G)$, the quasi-category $\operatorname{Sp}(G)$ admits all small limits by [27, Cor. 4.2.4.8]. By Lemma 6.2, the limit of any $U$-split cosimplicial object of $\operatorname{Sp}(G)$ is preserved by $U$. This completes the proof.

The following lemma is useful to show that other adjunctions are comonadic.

Lemma 6.4 Suppose we have a commutative diagram of quasi-categories

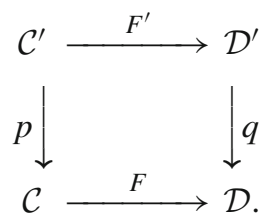

We assume that $F$ and $F^{\prime}$ are left adjoint functors, and that $p$ and $q$ are conservative. Furthermore, we assume that, for any cosimplicial object $X^{\bullet}$ in $\mathcal{C}^{\prime}$, if $p\left(X^{\bullet}\right)$ admits a limit in $\mathcal{C}$, then $X^{\bullet}$ admits a limit in $\mathcal{C}^{\prime}$ and that limit is preserved by $p$. If $F$ exhibits $\mathcal{C}$ as comonadic over $\mathcal{D}$, then $F^{\prime}$ exhibits $\mathcal{C}^{\prime}$ as comonadic over $\mathcal{D}^{\prime}$.

Proof We shall use the quasi-categorical Barr-Beck theorem by Lurie [28, 4.7.4]. We have to show that $F^{\prime}$ is conservative, $\mathcal{C}^{\prime}$ admits a limit for any $F^{\prime}$-split cosimplicial object, and the limit of any $F^{\prime}$-split cosimplicial object of $\mathcal{C}^{\prime}$ is preserved by $F^{\prime}$.

First, we show that $F^{\prime}$ is conservative. Since $F$ exhibits $\mathcal{C}$ as comonadic over $\mathcal{D}$, $F$ is conservative. Combining this with the conservativeness of $p$, we see that $F^{\prime}$ is conservative as well.

Next, we let $X^{\bullet}$ be an $F^{\prime}$-split cosimplicial object of $\mathcal{C}^{\prime}$. By applying $p$, we obtain an $F$-split cosimplicial object $p\left(X^{\bullet}\right)$ in $\mathcal{C}$ since split cosimplicial objects are preserved by any functor. Since $F$ exhibits $\mathcal{C}$ as comonadic over $\mathcal{D}, p\left(X^{\bullet}\right)$ admits a limit and that limit is preserved by $F$. By the assumption, $X^{\bullet}$ admits a limit and the limit is preserved by $p$. We see that the limit of $X^{\bullet}$ is preserved by $F^{\prime}$ since the composition $F p$ preserves the limit and $q$ is conservative.

Next, we consider the localized version of the adjunction $U_{k}: \operatorname{Sp}(G)_{k} \rightleftarrows \operatorname{Sp}_{k}: V_{k}$ and would like to show that the forgetful functor $U_{k}: \operatorname{Sp}(G)_{k} \rightarrow \operatorname{Sp}_{k}$ exhibits $\operatorname{Sp}(G)_{k}$ as comonadic over $\mathrm{Sp}_{k}$. For this purpose, we consider the following assumption on the localization functor $L_{k}$. 
Assumption 6.5 ( $c f$. [4, Assumption 1.0.3]) The localization functor $L_{k}$ on the stable homotopy category of (non-equivariant) spectra is given as a composite of two localization functors $L_{M} L_{T}$, where $L_{T}$ is a smashing localization and $L_{M}$ is a localization with respect to a finite spectrum $M$.

We note that the $K(n)$-localization $L_{K(n)}$ satisfies Assumption 6.5, where $K(n)$ is the $n$th Morava $K$-theory at a prime $p$. Let $E(n)$ be the $n$th Johnson-Wilson spectrum and $F(n)$ a finite spectrum of type $n$ at $p$. The $E(n)$-localization $L_{E(n)}$ is smashing by [33, Thm. 7.5.6], and the $K(n)$-localization is given as the composite $L_{F(n)} L_{E(n)}$ (see, for example, [23, Prop. 7.10]).

In order to compare the comonadicity of $\Sigma \operatorname{Sp}(G)_{k}$ and $\Sigma \operatorname{Sp}(G)$, we consider the functor

$$
M \wedge(-): \operatorname{Sp}(G)_{k} \rightarrow \operatorname{Sp}(G)
$$

given by smashing with a (non-equivariant) finite spectrum $M$. The functor $M \wedge(-)$ is right adjoint to the functor $(D M \wedge(-))_{k}$, where $D M$ is the $S$-dual of $M$ and $(-)_{k}$ is the $k$-localization functor on $\operatorname{Sp}(G)$. We shall show that $M \wedge(-)$ is conservative if the localization functor $L_{k}$ satisfies $L_{M} L_{k} \simeq L_{k}$.

Lemma 6.6 If the localization functor $L_{k}$ satisfies $L_{M} L_{k} \simeq L_{k}$ for a finite spectrum $M$, then the functor $M \wedge(-): \operatorname{Sp}(G)_{k} \rightarrow \operatorname{Sp}(G)$ is conservative.

Proof Suppose $f: X \rightarrow Y$ is a map in $\operatorname{Sp}(G)_{k}$ such that $M \wedge f$ is an equivalence in $\operatorname{Sp}(G)$. We have to show that $U f$ is a $k$-equivalence, where $U: \operatorname{Sp}(G)_{k} \rightarrow \operatorname{Sp}$ is the forgetful functor. Since $M$ is finite and $M \wedge U f$ is an equivalence, $M \wedge L_{k} U f \simeq$ $L_{k}(M \wedge U f)$ is also an equivalence. Hence $L_{M} L_{k} U f \simeq L_{k} U f$ is an equivalence. This completes the proof.

The following theorem shows that $\operatorname{Sp}(G)_{k}$ is comonadic over $\operatorname{Sp}_{k}$ under some conditions.

Theorem 6.7 If $G$ has finite virtual cohomological dimension and the localization functor $L_{k}$ satisfies Assumption 6.5, then the forgetful functor $U: \operatorname{Sp}(G)_{k} \rightarrow \operatorname{Sp}_{k}$ exhibits $\operatorname{Sp}(G)_{k}$ as comonadic over $\mathrm{Sp}_{k}$, that is, we have an equivalence of quasicategories

$$
\operatorname{Sp}(G)_{k} \stackrel{\simeq}{\longrightarrow} \operatorname{Comod}_{\Gamma}\left(\operatorname{Sp}_{k}\right)
$$

Proof Suppose $L_{k} \simeq L_{M} L_{T}$, where $M$ is a finite spectrum and $L_{T}$ is smashing. We shall apply Lemma 6.4 for the following diagram

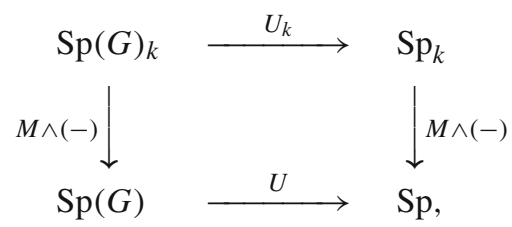


where the vertical arrows are given by smashing with the finite spectrum $M$.

First, we have to show that the diagram is commutative. Let $X$ be an object of $\operatorname{Sp}(G)_{k}$ which is represented by a fibrant and cofibrant object $Y$ in $\Sigma \operatorname{Sp}(G)_{k}$. Since $U_{k} X$ in $\mathrm{Sp}_{k}$ is represented by $L_{k} U Y$ in $\Sigma \mathrm{Sp}$, we have to show that the natural map $M \wedge U Y \rightarrow M \wedge L_{k} U Y$ is a weak equivalence in $\Sigma \mathrm{Sp}$. This follows from the assumption that $L_{k} \simeq L_{M} L_{T}$ and the fact that $U Y$ is $T$-local by [4, Prop. 6.1.7(2)].

The horizontal arrows are left adjoint functors, and the vertical arrows are conservative by Lemma 6.6. Since $\operatorname{Sp}(G)_{k}$ is the underlying quasi-category of the combinatorial simplicial model category $\Sigma \operatorname{Sp}(G)_{k}$, it admits all small limits by [27, Cor. 4.2.4.8]. Since $M \wedge(-): \operatorname{Sp}(G)_{k} \rightarrow \mathrm{Sp}_{k}$ is right adjoint, it preserves all limits by [27, Prop. 5.2.3.5]. By Proposition 6.3, $U$ exhibits $\operatorname{Sp}(G)$ as comonadic over Sp. Hence, by Lemma 6.4, $U_{k}$ exhibits $\operatorname{Sp}(G)_{k}$ as comonadic over $\operatorname{Sp}_{k}$.

\subsection{The quasi-category of algebra objects in $\operatorname{Sp}(G)_{k}$}

In this subsection we shall compare the quasi-category of algebra objects in $\operatorname{Sp}(G)_{k}$ with the underlying quasi-category of the simplicial model category of monoid objects in $\Sigma \operatorname{Sp}(G)_{k}$. Furthermore, we shall show that the quasi-category of algebra objects in $\operatorname{Sp}(G)_{k}$ is comonadic over the quasi-category of algebra objects in $\mathrm{Sp}_{k}$.

First, we recall a model structure on the category of monoid objects in $\Sigma \operatorname{Sp}(G)_{k}$. We denote by $\operatorname{Alg}\left(\Sigma \operatorname{Sp}(G)_{k}\right)$ the category of monoid objects in $\Sigma \operatorname{Sp}(G)_{k}$, and let $F: \operatorname{Alg}\left(\Sigma \operatorname{Sp}(G)_{k}\right) \rightarrow \Sigma \operatorname{Sp}(G)_{k}$ be the forgetful functor. By [35, Thm. 4.1(3)], $\operatorname{Alg}\left(\Sigma \operatorname{Sp}(G)_{k}\right)$ supports a model structure as follows. A map $f: X \rightarrow Y$ in $\operatorname{Alg}\left(\Sigma \operatorname{Sp}(G)_{k}\right)$ is said to be

- a weak equivalence if $F(f)$ is a weak equivalence in $\Sigma \operatorname{Sp}(G)_{k}$,

- a fibration if $F(f)$ is a fibration in $\Sigma \operatorname{Sp}(G)_{k}$, and

- a cofibration if it has the right lifting property with respect to all maps which are both fibrations and weak equivalences.

By Proposition 3.6, Theorem 3.7 and Proposition 3.8, $\Sigma \operatorname{Sp}(G)_{k}$ is a combinatorial symmetric monoidal simplicial model category which satisfies the monoid axiom. We see that $\operatorname{Alg}\left(\Sigma \operatorname{Sp}(G)_{k}\right)$ is a simplicial model category and the forgetful functor $F: \operatorname{Alg}\left(\Sigma \operatorname{Sp}(G)_{k}\right) \rightarrow \Sigma \operatorname{Sp}(G)_{k}$ is a simplicial right Quillen functor by [28, 4.1.4].

We compare the quasi-category of algebra objects in $\operatorname{Sp}(G)_{k}$ with the underlying quasi-category of the simplicial model category $\operatorname{Alg}\left(\Sigma \operatorname{Sp}(G)_{k}\right)$. Let $\operatorname{Alg}\left(\operatorname{Sp}(G)_{k}\right)$ be the quasi-category of algebra objects in $\operatorname{Sp}(G)_{k}$. By [28, 1.3.4 and 4.1.4], there is an equivalence of quasi-categories

$$
N\left(\operatorname{Alg}\left(\Sigma \operatorname{Sp}(G)_{k}\right)^{\circ}\right) \simeq \operatorname{Alg}\left(\operatorname{Sp}(G)_{k}\right)
$$

where $\operatorname{Alg}\left(\Sigma \operatorname{Sp}(G)_{k}\right)^{\circ}$ is the full simplicial subcategory of $\operatorname{Alg}\left(\Sigma \operatorname{Sp}(G)_{k}\right)$ consisting of objects that are both fibrant and cofibrant, and $N(-)$ is the simplicial nerve functor.

The forgetful functor $U: \Sigma \operatorname{Sp}(G)_{k} \rightarrow \Sigma \operatorname{Sp}_{k}$ induces a functor $U$ : $\operatorname{Alg}\left(\Sigma \operatorname{Sp}(G)_{k}\right) \rightarrow \operatorname{Alg}\left(\Sigma \mathrm{Sp}_{k}\right)$. We construct a right adjoint to the functor $U$ : $\operatorname{Alg}\left(\Sigma \operatorname{Sp}(G)_{k}\right) \rightarrow \operatorname{Alg}\left(\Sigma \operatorname{Sp}_{k}\right)$. For $Y \in \operatorname{Alg}\left(\Sigma \operatorname{Sp}_{k}\right)$, we have an object $\operatorname{Map}_{c}(G, Y)$ in $\Sigma \operatorname{Sp}(G)_{k}$. We consider a map 


$$
\operatorname{Map}_{c}(G, Y) \wedge \operatorname{Map}_{c}(G, Y) \longrightarrow \operatorname{Map}_{c}(G, Y)
$$

in $\Sigma \operatorname{Sp}(G)_{k}$, which is the adjoint to the map

$$
\begin{aligned}
& U\left(\operatorname{Map}_{c}(G, Y) \wedge \operatorname{Map}_{c}(G, Y)\right) \\
& \quad \cong U\left(\operatorname{Map}_{c}(G, Y)\right) \wedge U\left(\operatorname{Map}_{c}(G, Y)\right) \stackrel{\operatorname{ev}(e) \wedge \operatorname{ev}(e)}{\longrightarrow} Y \wedge Y \stackrel{m}{\longrightarrow} Y,
\end{aligned}
$$

where ev $(e)$ is the evaluation map at the identity element $e \in G$ and $m$ is the multiplica-

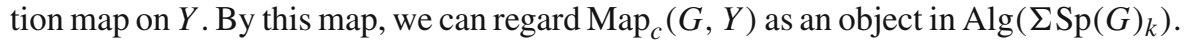
Hence we obtain a functor

$$
V: \operatorname{Alg}\left(\Sigma \operatorname{Sp}_{k}\right) \longrightarrow \operatorname{Alg}\left(\Sigma \operatorname{Sp}(G)_{k}\right)
$$

given by $V(Y)=\operatorname{Map}_{c}(G, Y)$. We see that $V$ is a right adjoint to the forgetful functor $U$.

Proposition 6.8 The adjoint pair of functors

$$
U: \operatorname{Alg}\left(\Sigma \operatorname{Sp}(G)_{k}\right) \rightleftarrows \operatorname{Alg}\left(\Sigma \operatorname{Sp}_{k}\right): V
$$

is a simplicial Quillen adjunction.

Proof Let $F: \operatorname{Alg}\left(\Sigma \operatorname{Sp}(G)_{k}\right) \rightarrow \Sigma \operatorname{Sp}(G)_{k}$ be the forgetful functor. We consider the following commutative diagram

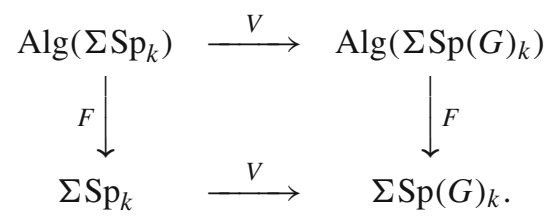

By Proposition 3.10, $V: \Sigma \mathrm{Sp}_{k} \rightarrow \Sigma \mathrm{Sp}(G)_{k}$ is a right Quillen functor. This implies that $V: \operatorname{Alg}\left(\Sigma \mathrm{Sp}_{k}\right) \rightarrow \operatorname{Alg}\left(\Sigma \operatorname{Sp}(G)_{k}\right)$ is also a right Quillen functor.

By Proposition 6.8, we have an adjunction of quasi-categories

$$
U_{k}: \operatorname{Alg}\left(\operatorname{Sp}(G)_{k}\right) \rightleftarrows \operatorname{Alg}\left(\operatorname{Sp}_{k}\right): V_{k}
$$

This induces a map of quasi-categories

$$
\operatorname{Alg}\left(\operatorname{Sp}(G)_{k}\right) \longrightarrow \operatorname{Comod}_{\Gamma}\left(\operatorname{Alg}\left(\operatorname{Sp}_{k}\right)\right)
$$

where $\Gamma$ is the comonad on $\operatorname{Alg}\left(\operatorname{Sp}_{k}\right)$ associated to the adjunction $\left(U_{k}, V_{k}\right)$. The following theorem shows that $\operatorname{Alg}\left(\operatorname{Sp}(G)_{k}\right)$ is comonadic over $\operatorname{Alg}\left(\operatorname{Sp}_{k}\right)$. 
Theorem 6.9 Let $G$ be a profinite group that has finite virtual cohomological dimension. We assume that the localization functor $L_{k}$ satisfies Assumption 6.5. Then the forgetful functor $U_{k}: \operatorname{Alg}\left(\operatorname{Sp}(G)_{k}\right) \rightarrow \operatorname{Alg}\left(\operatorname{Sp}_{k}\right)$ exhibits $\operatorname{Alg}\left(\operatorname{Sp}(G)_{k}\right)$ as comonadic over $\operatorname{Alg}\left(\mathrm{Sp}_{k}\right)$, that is, we have an equivalence of quasi-categories

$$
\operatorname{Alg}\left(\operatorname{Sp}(G)_{k}\right) \stackrel{\simeq}{\longrightarrow} \operatorname{Comod}_{\Gamma}\left(\operatorname{Alg}\left(\operatorname{Sp}_{k}\right)\right) \text {. }
$$

Proof We shall apply Lemma 6.4 for the commutative diagram

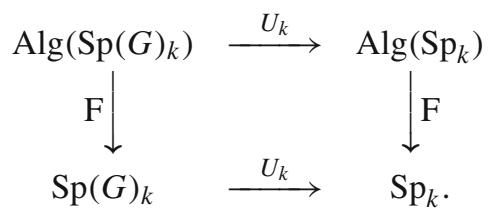

The horizontal arrows are left adjoint functors and the vertical arrows are conservative. Since $\operatorname{Alg}\left(\Sigma \operatorname{Sp}(G)_{k}\right)$ is a combinatorial simplicial model category and $\operatorname{Alg}\left(\operatorname{Sp}(G)_{k}\right)$ is its underlying quasi-category, $\operatorname{Alg}\left(\operatorname{Sp}(G)_{k}\right)$ admits all small limits by [27, Cor. 4.2.4.8]. Since the forgetful functor $F: \operatorname{Alg}\left(\operatorname{Sp}(G)_{k}\right) \rightarrow \operatorname{Sp}(G)_{k}$ is right adjoint, it preserves limits by [27, Prop. 5.2.3.5]. By Theorem 6.7, $U_{k}: \operatorname{Sp}(G)_{k} \rightarrow \operatorname{Sp}_{k}$ exhibits $\operatorname{Sp}(G)_{k}$ as comonadic over $\mathrm{Sp}_{k}$. Hence the theorem follows from Lemma 6.4.

\subsection{The quasi-category of module objects in $\operatorname{Sp}(G)_{k}$}

In this subsection we shall show that the quasi-category of module objects in $\operatorname{Sp}(G)_{k}$ is comonadic over the quasi-category of module objects in $\mathrm{Sp}_{k}$.

First, we compare the quasi-category of module objects in $\operatorname{Sp}(G)_{k}$ and the underlying quasi-category of the simplicial model category of module objects in $\Sigma \operatorname{Sp}(G)_{k}$. Let $B$ be a monoid object in $\Sigma \operatorname{Sp}(G)_{k}$. We assume that $B$ is cofibrant in $\Sigma \operatorname{Sp}(G)_{k}$. We denote by $U B$ the underlying monoid object in $\Sigma \operatorname{Sp}_{k}$. Note that $U B$ is cofibrant in $\Sigma \mathrm{Sp}_{k}$ by Proposition 3.10. By [28, 1.3.4 and 4.3.3], the underlying quasicategories of $\operatorname{Mod}_{B}\left(\Sigma \operatorname{Sp}(G)_{k}\right)$ and $\operatorname{Mod}_{U B}\left(\Sigma \operatorname{Sp}_{k}\right)$ are equivalent to $\operatorname{Mod}_{B}\left(\operatorname{Sp}(G)_{k}\right)$ and $\operatorname{Mod}_{U B}\left(\operatorname{Sp}_{k}\right)$, respectively.

We have the forgetful functor $U: \operatorname{Mod}_{B}\left(\Sigma \operatorname{Sp}(G)_{k}\right) \rightarrow \operatorname{Mod}_{U B}\left(\Sigma \operatorname{Sp}_{k}\right)$. We shall construct a right adjoint to $U$. For $M \in \operatorname{Mod}_{U B}\left(\Sigma \operatorname{Sp}_{k}\right)$, we $\operatorname{regard} \operatorname{Map}_{c}(G, M)$ as an object in $\Sigma \operatorname{Sp}(G)_{k}$. We consider a map

$$
B \wedge \operatorname{Map}_{c}(G, M) \longrightarrow \operatorname{Map}_{c}(G, M)
$$

in $\Sigma \operatorname{Sp}(G)_{k}$, which is an adjoint to the map

$$
U\left(B \wedge \operatorname{Map}_{c}(G, M)\right) \cong U B \wedge U \operatorname{Map}_{c}(G, M) \stackrel{\operatorname{id} \wedge \operatorname{ev}(e)}{\longrightarrow} U B \wedge M \stackrel{a}{\longrightarrow} M,
$$

where $\operatorname{ev}(e)$ is the evaluation map at the identity element $e \in G$ and $a$ is the action map on $M$. This defines a $B$-module structure on $\operatorname{Map}_{c}(G, M)$ and we see that $\operatorname{Map}_{c}(G, M)$ is an object in $\operatorname{Mod}_{B}\left(\Sigma \operatorname{Sp}(G)_{k}\right)$. Hence we obtain a functor 


$$
V: \operatorname{Mod}_{U B}\left(\Sigma \mathrm{Sp}_{k}\right) \longrightarrow \operatorname{Mod}_{B}\left(\Sigma \operatorname{Sp}(G)_{k}\right)
$$

given by $V(M)=\operatorname{Map}_{c}(G, M)$. We see that $V$ is a right adjoint to the forgetful functor $U$, and hence we have an adjunction

$$
U: \operatorname{Mod}_{B}\left(\Sigma \operatorname{Sp}(G)_{k}\right) \rightleftarrows \operatorname{Mod}_{U B}\left(\Sigma \operatorname{Sp}_{k}\right): V
$$

Lemma 6.10 The adjoint pair $(U, V)$ is a $\Sigma \mathrm{Sp}$-Quillen adjunction.

Proof This follows from Proposition 3.10.

The $\Sigma$ Sp-Quillen adjunction $(U, V)$ induces an adjunction of quasi-categories

$$
U_{k}: \operatorname{Mod}_{B}\left(\operatorname{Sp}(G)_{k}\right) \rightleftarrows \operatorname{Mod}_{U B}\left(\operatorname{Sp}_{k}\right): V_{k}
$$

Let $\Gamma$ be the comonad on $\operatorname{Mod}_{U B}\left(\operatorname{Sp}_{k}\right)$ associated to the adjoint pair $\left(U_{k}, V_{k}\right)$, and let

$$
\operatorname{Comod}_{(U B, \Gamma)}\left(\operatorname{Sp}_{k}\right)=\operatorname{Comod}_{\Gamma}\left(\operatorname{Mod}_{U B}\left(\operatorname{Sp}_{k}\right)\right)
$$

be the quasi-category of comodules over $\Gamma$. The following theorem shows that $\operatorname{Mod}_{B}\left(\operatorname{Sp}(G)_{k}\right)$ is comonadic over $\operatorname{Mod}_{U B}\left(\operatorname{Sp}_{k}\right)$ under some conditions.

Theorem 6.11 Let $G$ be a profinite group that has finite virtual cohomological dimension. We assume that the localization functor $L_{k}$ satisfies Assumption 6.5. Then the forgetful functor $U_{k}: \operatorname{Mod}_{B}\left(\operatorname{Sp}(G)_{k}\right) \rightarrow \operatorname{Mod}_{U B}\left(\operatorname{Sp}_{k}\right)$ exhibits $\operatorname{Mod}_{B}\left(\operatorname{Sp}(G)_{k}\right)$ as comonadic over $\operatorname{Mod}_{U B}\left(\operatorname{Sp}_{k}\right)$, that is, we have an equivalence of quasi-categories

$$
\operatorname{Mod}_{B}\left(\operatorname{Sp}(G)_{k}\right) \stackrel{\simeq}{\longrightarrow} \operatorname{Comod}_{(U B, \Gamma)}\left(\operatorname{Sp}_{k}\right)
$$

Proof The theorem follows in the same way as the proof of Theorem 6.9 by applying Lemma 6.4 for the commutative diagram

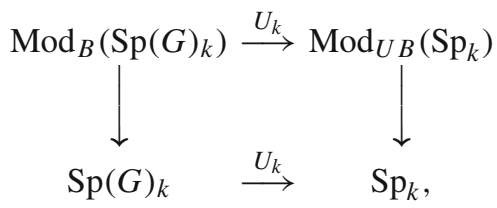

where the vertical arrows are forgetful functors.

\subsection{Equivalence of the two formulations}

In this subsection we shall show that Propositions 4.5 and 5.2 are equivalent in some circumstances.

First, we recall the relationship between functors of $\Sigma \mathrm{Sp}$-model categories and of the underlying quasi-categories. The homotopy category of the underlying quasicategory of a $\Sigma$ Sp-model category $\mathbf{M}$ is equivalent to the homotopy category of $\mathbf{M}$ as Ho( $\Sigma$ Sp)-enriched categories. Let 


$$
F: \mathbf{M} \rightleftarrows \mathbf{N}: G
$$

be a $\Sigma$ Sp-Quillen adjunction between combinatorial $\Sigma$ Sp-model categories. The adjunction $(F, G)$ induces an adjunction

$$
\mathcal{F}: \mathcal{M} \rightleftarrows \mathcal{N}: \mathcal{G}
$$

of quasi-categories, where $\mathcal{M}$ and $\mathcal{N}$ are the underlying quasi-categories of $\mathbf{M}$ and $\mathbf{N}$, respectively. The adjunction $(\mathcal{F}, \mathcal{G})$ induces an adjunction

$$
\operatorname{Ho}(\mathcal{F}): \operatorname{Ho}(\mathcal{M}) \rightleftarrows \operatorname{Ho}(\mathcal{N}): \operatorname{Ho}(G)
$$

on the homotopy categories. The adjunction $(\operatorname{Ho}(\mathcal{F}), \operatorname{Ho}(\mathcal{G}))$ is identified with the derived adjunction $(\mathbb{L} F, \mathbb{R} G)$ under the equivalences $\operatorname{Ho}(\mathbf{M}) \simeq \operatorname{Ho}(\mathcal{M})$ and $\operatorname{Ho}(\mathbf{N}) \simeq$ $\operatorname{Ho}(\mathcal{N})$.

Since $\mathbf{M}$ is a $\Sigma$ Sp-model category, $\mathbf{M}$ is a stable model category by [36, Lem. 3.5.2], and hence $\mathrm{Ho}(\mathbf{M})$ is a triangulated category. Suppose that $\mathbf{T}$ is a triangulated subcategory of $\operatorname{Ho}(\mathbf{M})$. We have the corresponding full subcategory $\mathcal{T}$ of $\mathcal{M}$. The total left derived functor $\mathbb{L}(F): \operatorname{Ho}(\mathbf{M}) \rightarrow \operatorname{Ho}(\mathbf{N})$ restricted to $\mathbf{T}$ is fully faithful as an $\operatorname{Ho}(\Sigma \mathrm{Sp})$-enriched functor if and only if $\mathcal{F}: \mathcal{M} \rightarrow \mathcal{N}$ restricted to $\mathcal{T}$ is fully faithful.

Let $A$ be a monoid object in $\Sigma \mathrm{Sp}$. We regard $A$ as a monoid object in $\Sigma \operatorname{Sp}(G)$ with trivial $G$ action. Let $\varphi: A \rightarrow B$ be a map of monoid objects in $\Sigma \operatorname{Sp}(G)$. We assume that $A$ is cofibrant in $\Sigma$ Sp and that $U B$ is fibrant and cofibrant in $\Sigma$ Sp. We have the $\Sigma$ Sp-Quillen adjunction

$$
\operatorname{Ex}: \operatorname{Mod}_{A}\left(\Sigma \operatorname{Sp}_{k}\right) \rightleftarrows \operatorname{Mod}_{B}\left(\Sigma \operatorname{Sp}(G)_{k}\right): \operatorname{Re},
$$

which induces the adjunction $\mathbb{L E x}: \operatorname{Ho}\left(\operatorname{Mod}_{A}\left(\Sigma \operatorname{Sp}_{k}\right)\right) \rightleftarrows \operatorname{Ho}\left(\operatorname{Mod}_{B}\left(\Sigma \operatorname{Sp}(G)_{k}\right)\right)$ : $\mathbb{R} R$ e of the homotopy categories. The $\Sigma$ Sp-Quillen adjunction $(\mathrm{Ex}, \mathrm{Re})$ also induces the adjunction of quasi-categories

$$
\mathcal{E} x: \operatorname{Mod}_{A}\left(\operatorname{Sp}_{k}\right) \rightleftarrows \operatorname{Mod}_{B}\left(\operatorname{Sp}(G)_{k}\right): \mathcal{R} e .
$$

We can regard this adjunction as a lifting of the adjunction ( $\mathbb{L E x}, \mathbb{R} R e)$.

On the other hand, we have the adjunction of quasi-categories

$$
U_{k}: \operatorname{Mod}_{A}\left(\operatorname{Sp}_{k}\right) \rightleftarrows \operatorname{Mod}_{U B}\left(\operatorname{Sp}_{k}\right): V_{k},
$$

which induces the adjunction of quasi-categories

$$
\text { Coex : } \operatorname{Mod}_{A}\left(\operatorname{Sp}_{k}\right) \rightleftarrows \operatorname{Comod}_{(U B, \Theta)}\left(\operatorname{Sp}_{k}\right): P \text {. }
$$

In this subsection, under some conditions, we shall show that $\operatorname{Comod}_{(U B, \Theta)}\left(\operatorname{Sp}_{k}\right)$ is equivalent to $\operatorname{Mod}_{B}\left(\operatorname{Sp}(G)_{k}\right)$ and the functor $\mathcal{E} x$ is equivalent to Coex under this equivalence. This implies that the right adjoint $\mathcal{R} e$ is equivalent to $P$, and the full subcategory $\mathcal{T}$ of $\operatorname{Mod}_{A}\left(\operatorname{Sp}_{k}\right)$ corresponds to the thick subcategory $\mathbf{T}$ of $\operatorname{Ho}\left(\operatorname{Mod}_{A}\left(\Sigma \operatorname{Sp}_{k}\right)\right)$, where 
$\mathcal{T}$ is the full subcategory of $\operatorname{Mod}_{A}\left(\operatorname{Sp}_{k}\right)$ consisting of objects $X$ such that the unit map $X \rightarrow P \operatorname{Coex}(X)$ is an equivalence and the thick subcategory $\mathbf{T}$ of $\operatorname{Ho}\left(\operatorname{Mod}_{A}\left(\Sigma \operatorname{Sp}_{k}\right)\right)$ consists of objects $Y$ such that the unit map $Y \rightarrow \mathbb{R} \operatorname{Re} \mathbb{L E x}(Y)$ is an isomorphism in the homotopy category. This means that the formulation of embeddings of module categories in terms of model categories in Proposition 4.5 and that in terms of quasi-categories in Proposition 5.2 are equivalent.

We begin with constructing a map which compares the two-sided bar construction with the functor $\operatorname{Map}_{c}(G,-)$. For $M \in \operatorname{Mod}_{U B}(\Sigma \mathrm{Sp})$, we denote by

$$
B(U B, A, M)=\left|B_{\bullet}(U B, A, M)\right|
$$

the geometric realization of the bar construction $B_{\bullet}(U B, A, M)$. We define a map

$$
\Psi_{M}: B(U B, A, M) \longrightarrow U \operatorname{Map}_{c}(G, M)
$$

by applying $U$ to the map $B(B, A, M) \rightarrow \operatorname{Map}_{c}(G, M)$ in $\Sigma \operatorname{Sp}(G)$ that is adjoint to the map $U B(B, A, M) \cong B(U B, A, M) \rightarrow M$ induced by the action of $U B$ on $M$. In particular, we have a map

$$
\Psi_{U B}: B(U B, A, U B) \longrightarrow U \operatorname{Map}_{c}(G, U B) .
$$

We set $\psi=U \varphi: A \rightarrow U B$. Recall that there is a Quillen adjunction

$$
U B \wedge_{A}(-): \operatorname{Mod}_{A}\left(\Sigma \operatorname{Sp}_{k}\right) \rightleftarrows \operatorname{Mod}_{U B}\left(\Sigma \operatorname{Sp}_{k}\right): \psi^{*}
$$

which induces an adjunction

$$
U B \wedge_{A}^{\mathbb{L}}(-): \operatorname{Ho}\left(\operatorname{Mod}_{A}\left(\Sigma \operatorname{Sp}_{k}\right)\right) \rightleftarrows \operatorname{Ho}\left(\operatorname{Mod}_{U B}\left(\Sigma \operatorname{Sp}_{k}\right)\right): \mathbb{R} \psi^{*}
$$

between the homotopy categories, where $U B \wedge_{A}^{\mathbb{L}}(-)$ is the total left derived functor of $U B \wedge_{A}(-)$ and $\mathbb{R} \psi^{*}$ is the total right derived functor of $\psi^{*}$. The following lemma shows that the bar construction $B(U B, A,-)$ is a model of the composition $U B \wedge_{A}^{\mathbb{L}}$ $\left(\mathbb{R} \psi^{*}(-)\right)$ of the functors.

Lemma 6.12 If $M$ is a fibrant and cofibrant object in $\operatorname{Mod}_{U B}\left(\Sigma \operatorname{Sp}_{k}\right)$, then $B(U B, A, M)$ represents $U B \wedge_{A}^{\mathbb{L}}\left(\mathbb{R} \psi^{*} M\right)$ in $\operatorname{Ho}\left(\operatorname{Mod}_{U B}\left(\Sigma \operatorname{Sp}_{k}\right)\right)$.

Proof Since $M$ is fibrant in $\operatorname{Mod}_{U B}\left(\Sigma \operatorname{Sp}_{k}\right), M$ represents $\mathbb{R} \psi^{*} M$ in $\operatorname{Ho}\left(\operatorname{Mod}_{A}\left(\Sigma \operatorname{Sp}_{k}\right)\right)$. If $Q_{A} M \rightarrow M$ is a cofibrant replacement in $\operatorname{Mod}_{A}\left(\Sigma \operatorname{Sp}_{k}\right)$, then $U B \wedge_{A}^{\mathbb{L}}\left(\mathbb{R} \psi^{*} M\right)$ is represented by $U B \wedge_{A} Q_{A} M$. By [37, Lem. 4.1.9], we have an equivalence $B\left(U B, A, Q_{A} M\right) \stackrel{\simeq}{\rightarrow} U B \wedge_{A} Q_{A} M$.

We shall show that there is an equivalence $B\left(U B, A, Q_{A} M\right) \stackrel{\simeq}{\rightarrow} B(U B, A, M)$. For any $r \geq 0, U B \wedge A^{\wedge r}$ is cofibrant in $\Sigma$ Sp. This implies an equivalence $U B \wedge$ $A^{\wedge r} \wedge Q_{A} M \stackrel{\simeq}{\rightarrow} U B \wedge A^{\wedge r} \wedge M$ by [24, Lem. 5.4.4]. Hence we obtain an equivalence $\left|B_{\bullet}\left(U B, A, Q_{A} M\right)\right| \stackrel{\simeq}{\rightarrow}\left|B_{\bullet}(U B, A, M)\right|$ by $[37$, Cor. 4.1.6]. 
The following lemma shows that it suffices to show that $\Psi_{U B}$ is a $k$-local equivalence in order to ensure that $\Psi_{M}$ is a $k$-local equivalence.

Lemma 6.13 Let $M$ be a cofibrant and fibrant object in $\operatorname{Mod}_{U B}\left(\Sigma \operatorname{Sp}_{k}\right)$. If $\Psi_{U B}$ is a $k$-local equivalence, then $\Psi_{M}$ is also a $k$-local equivalence.

Proof We have an isomorphism between $B(U B, A, M)$ and $B(U B, A, U B) \wedge_{U B} M$, and an equivalence between $U \operatorname{Map}_{c}(G, M)$ and $U \operatorname{Map}_{c}(G, U B) \wedge U B M$. Since $M$ is cofibrant in $\operatorname{Mod}_{U B}(\Sigma \mathrm{Sp})$, the $k$-local equivalence $\Psi_{U B}$ induces a $k$-local equivalence

$$
B(U B, A, U B) \wedge_{U B} M \stackrel{\simeq_{k}}{\longrightarrow} U \operatorname{Map}_{c}(G, U B) \wedge_{U B} M
$$

by [24, Lem. 5.4.4]. This completes the proof.

There is an adjunction of quasi-categories

$$
U B \wedge_{A}(-): \operatorname{Mod}_{A}\left(\operatorname{Sp}_{k}\right) \rightleftarrows \operatorname{Mod}_{U B}\left(\operatorname{Sp}_{k}\right): \psi^{*}
$$

and hence we obtain a comonad $\Theta$ on $\operatorname{Mod}_{U B}\left(\operatorname{Sp}_{k}\right)$ and a quasi-category of comodules

$$
\operatorname{Comod}_{(U B, \Theta)}\left(\operatorname{Sp}_{k}\right)=\operatorname{Comod}_{\Theta}\left(\operatorname{Mod}_{U B}\left(\operatorname{Sp}_{k}\right)\right)
$$

To ease notation, we set

$$
\begin{aligned}
& \mathcal{C}=\operatorname{Mod}_{U B}\left(\operatorname{Sp}_{k}\right)^{\mathrm{op}}, \\
& \mathcal{C}(G)=\operatorname{Mod}_{B}\left(\operatorname{Sp}(G)_{k}\right)^{\mathrm{op}}, \\
& \mathcal{D}=\operatorname{Mod}_{A}\left(\operatorname{Sp}_{k}\right)^{\mathrm{op}} .
\end{aligned}
$$

We have an adjunction of quasi-categories $V: \mathcal{C} \rightleftarrows \mathcal{C}(G): U$. By [28, 4.7.4], there is an endomorphism monad of $U$, and hence we have a monad $\Gamma \in \operatorname{Alg}(\operatorname{End}(\mathcal{C}))$ and a left $\Gamma$-module $\bar{U} \in \operatorname{Mod}_{\Gamma}(\operatorname{Fun}(\mathcal{C}(G), \mathcal{C}))$. Note that $\Gamma$ is a lifting of $U V$ and $\bar{U}$ is a lifting of $U$.

We set

$$
\begin{aligned}
& H=U B \wedge_{A}(-): \mathcal{D} \rightarrow \mathcal{C}, \\
& H^{\prime}=B \wedge_{A}(-): \mathcal{D} \rightarrow \mathcal{C}(G) \\
& F=\psi^{*}: \mathcal{C} \rightarrow \mathcal{D}
\end{aligned}
$$

Note that $H=U H^{\prime}$ is the right adjoint to $F$. Hence there is an endomorphism monad of $H$, which consists of a monad $\Theta \in \operatorname{Alg}(\operatorname{End}(C))$ together with $\bar{H} \in$ $\operatorname{Mod}_{\Theta}(\operatorname{Fun}(\mathcal{D}, \mathcal{C}))$ that is a lifting of $H$. We note that the functor $H=U H^{\prime}$ lifts to a left $\Gamma$-module $\bar{U} H^{\prime} \in \operatorname{Mod}_{\Gamma}(\operatorname{Fun}(\mathcal{D}, \mathcal{C}))$.

We would like to show that the monad $\Gamma$ together with the left $\Gamma$-module object $\bar{U} H^{\prime}$ is an endomorphism monad of $H$. For this purpose, we consider the composite map

$$
\Gamma \stackrel{\operatorname{id}_{\Gamma} \times u}{\longrightarrow} \Gamma H F \stackrel{a \times \operatorname{id}_{F}}{\longrightarrow} H F
$$


where $u$ is the unit of the adjoint pair $(F, H)$ and $a$ is the action of $\Gamma$ on $H$. For any $M \in \operatorname{Mod}_{U B}\left(\operatorname{Sp}_{k}\right)$, this map induces a natural map

$$
U \operatorname{Map}_{c}(G, M) \longleftarrow U \operatorname{Map}_{c}\left(G, U B \wedge_{A} M\right) \longleftarrow U B \wedge_{A} M
$$

in $\operatorname{Mod}_{U B}\left(\operatorname{Sp}_{k}\right)$.

Lemma 6.14 If $\Psi_{U B}$ is a $k$-local equivalence, then the composite map $\Gamma \rightarrow \Gamma H F \rightarrow$ $H F$ is an equivalence of functors.

Proof It suffices to show that the induced map $U B \wedge_{A} M \longrightarrow U \operatorname{Map}_{c}(G, M)$ is an equivalence for any $M$ in $\operatorname{Mod}_{U B}\left(\operatorname{Sp}_{k}\right)$. This follows from Lemmas 6.12 and 6.13.

If $\Psi_{U B}$ is a $k$-local equivalence, by Lemma 6.14 and [28, 4.7.4], we see that the $\operatorname{monad} \Gamma \in \operatorname{Alg}(\operatorname{End}(\mathcal{C}))$ together with the object $\bar{U} H^{\prime} \in \operatorname{Mod}_{\Gamma}(\operatorname{Fun}(\mathcal{D}, \mathcal{C}))$ is an endomorphism monad of $H$. Hence we obtain an equivalence of quasi-categories

$$
\operatorname{Mod}_{\Theta}(\operatorname{Fun}(\mathcal{D}, \mathcal{C})) \stackrel{\simeq}{\longrightarrow} \operatorname{Mod}_{\Gamma}(\operatorname{Fun}(\mathcal{D}, \mathcal{C}))
$$

compatible with the forgetful functors to $\operatorname{Fun}(\mathcal{D}, \mathcal{C})$, and the object $\bar{H} \in \operatorname{Mod}_{\Theta}$ $(\operatorname{Fun}(\mathcal{D}, \mathcal{C}))$ corresponds to the object $\bar{U} H^{\prime} \in \operatorname{Mod}_{\Gamma}(\operatorname{Fun}(\mathcal{D}, \mathcal{C}))$ under this equivalence. Since the pair $\left(\Gamma, \bar{U} H^{\prime}\right)$ is an endomorphism monad of $H$, in particular, there is an equivalence $\Gamma \stackrel{\simeq}{\rightarrow} \Theta$ in $\operatorname{Alg}(\operatorname{End}(\mathcal{C}))$. This equivalence of algebra objects induces an equivalence

$$
\operatorname{Mod}_{\Theta}(\mathcal{E}) \stackrel{\simeq}{\longrightarrow} \operatorname{Mod}_{\Gamma}(\mathcal{E})
$$

for any quasi-category left-tensored over $\operatorname{End}(\mathcal{C})$. Taking $\mathcal{C}$ as $\mathcal{E}$, we obtain the following theorem.

Theorem 6.15 If $\Psi_{U B}$ is a $k$-local equivalence, then there is an equivalence of quasicategories

$$
\operatorname{Comod}_{(U B, \Gamma)}\left(\operatorname{Sp}_{k}\right) \simeq \operatorname{Comod}_{(U B, \Theta)}\left(\operatorname{Sp}_{k}\right)
$$

By Theorems 6.11 and 6.15, we obtain the following corollary.

Corollary 6.16 Let $G$ be a profinite group that has finite virtual cohomological dimension. We assume that the localization functor $L_{k}$ satisfies Assumption 6.5. If $\Psi_{U B}$ is a $k$-local equivalence, then there is an equivalence of quasi-categories

$$
\operatorname{Mod}_{B}\left(\operatorname{Sp}(G)_{k}\right) \simeq \operatorname{Comod}_{(U B, \Theta)}\left(\operatorname{Sp}_{k}\right)
$$

Now we would like to show that the two formulations of embeddings of module objects in terms of model categories and in terms of quasi-categories are equivalent under some conditions. 
First, we shall compare the $\operatorname{map} \mathcal{D} \rightarrow \operatorname{Mod}_{\Theta}(\mathcal{C})$ given by $\bar{H} \in \operatorname{Mod}_{\Theta}(\operatorname{Fun}(\mathcal{D}, \mathcal{C}))$ with the $\operatorname{map} \mathcal{C}(G) \rightarrow \operatorname{Mod}_{\Gamma}(\mathcal{C})$ given by $\bar{U} \in \operatorname{Mod}_{\Gamma}(\operatorname{Fun}(\mathcal{C}(G), \mathcal{C}))$. The evaluation functor $\mathcal{E} \times \operatorname{Fun}(\mathcal{E}, \mathcal{C}) \rightarrow \mathcal{C}$ is a map of quasi-categories left-tensored over $\operatorname{End}(\mathcal{C})$ for any quasi-category $\mathcal{E}$. This induces a map

$$
\mathcal{E} \times \operatorname{Mod}_{T}(\operatorname{Fun}(\mathcal{E}, \mathcal{C})) \simeq \operatorname{Mod}_{T}(\mathcal{E} \times \operatorname{Fun}(\mathcal{E}, \mathcal{C})) \longrightarrow \operatorname{Mod}_{T}(\mathcal{C})
$$

for any monad $T \in \operatorname{Alg}(\operatorname{End}(\mathcal{C}))$. By adjunction, we obtain a map

$$
d(\mathcal{E}, T): \operatorname{Mod}_{T}(\operatorname{Fun}(\mathcal{E}, \mathcal{C})) \longrightarrow \operatorname{Fun}\left(\mathcal{E}, \operatorname{Mod}_{T}(\mathcal{C})\right)
$$

which is an equivalence for any quasi-category $\mathcal{E}$.

We assume that $\Psi_{U B}$ is a $k$-local equivalence. In particular, we have an equivalence $\Gamma \stackrel{\simeq}{\rightarrow} \Theta$ in $\operatorname{Alg}(\operatorname{End}(\mathcal{C}))$. By the naturality of the construction, we obtain a commutative diagram

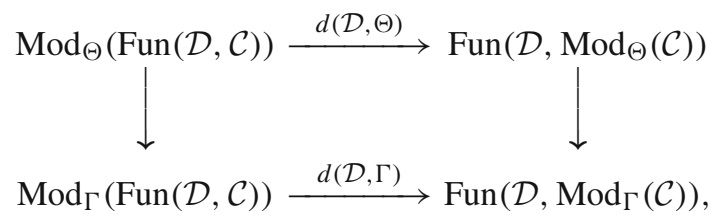

where all the arrows are equivalences. We denote by $\widetilde{H} \in \operatorname{Fun}\left(\mathcal{D}, \operatorname{Mod}_{\Theta}(\mathcal{C})\right)$ the image of $\bar{H} \in \operatorname{Mod}_{\Theta}(\operatorname{Fun}(\mathcal{D}, \mathcal{C}))$ under the map $d(\mathcal{D}, \Theta)$ and by $\widetilde{U} \in \operatorname{Fun}\left(\mathcal{C}(G), \operatorname{Mod}_{\Gamma}(\mathcal{C})\right)$ the image of $\bar{U} \in \operatorname{Mod}_{\Gamma}(\operatorname{Fun}(\mathcal{C}(G), \mathcal{C}))$ under the map $d(\mathcal{C}(G), \Gamma)$. Since $\bar{H}$ corresponds to $\bar{U} H^{\prime}$ under the equivalence $\operatorname{Mod}_{\Theta}(\operatorname{Fun}(\mathcal{D}, \mathcal{C})) \stackrel{\simeq}{\rightarrow} \operatorname{Mod}_{\Gamma}(\operatorname{Fun}(\mathcal{D}, \mathcal{C}))$, we see that $\widetilde{H}$ corresponds to $\widetilde{U} H^{\prime}$ under the equivalence $\operatorname{Fun}\left(\mathcal{D}, \operatorname{Mod}_{\Theta}(\mathcal{C})\right) \stackrel{\simeq}{\stackrel{\simeq}{\rightarrow}}$ $\operatorname{Fun}\left(\mathcal{D}, \operatorname{Mod}_{\Gamma}(\mathcal{C})\right)$ using commutative diagram (6.2).

The functor $\operatorname{Ex}=B \wedge_{A}(-): \operatorname{Mod}_{A}\left(\Sigma \operatorname{Sp}_{k}\right) \rightarrow \operatorname{Mod}_{B}\left(\Sigma \operatorname{Sp}(G)_{k}\right)$ of $\Sigma S p$-model categories induces a functor

$$
\mathcal{E} x: \operatorname{Mod}_{A}\left(\operatorname{Sp}_{k}\right) \rightarrow \operatorname{Mod}_{B}\left(\operatorname{Sp}(G)_{k}\right)
$$

of quasi-categories. We can identify the functor $\mathcal{E} x^{\text {op }}: \operatorname{Mod}_{A}\left(\operatorname{Sp}_{k}\right)^{\text {op }} \rightarrow$ $\operatorname{Mod}_{B}\left(\operatorname{Sp}(G)_{k}\right)$ op induced on the opposite quasi-categories with $H^{\prime}: \mathcal{D} \rightarrow \mathcal{C}(G)$. Recall that we have the functor

$$
\text { Coex }: \operatorname{Mod}_{A}\left(\operatorname{Sp}_{k}\right) \rightarrow \operatorname{Comod}_{(U B, \Theta)}\left(\operatorname{Sp}_{k}\right)
$$

of quasi-categories. We can identify the functor $\operatorname{Coex}^{\mathrm{op}}: \operatorname{Mod}_{A}\left(\operatorname{Sp}_{k}\right)^{\mathrm{op}} \rightarrow$ $\operatorname{Comod}_{(U B, \Theta)}\left(\operatorname{Sp}_{k}\right)^{\text {op }}$ induced on the opposite quasi-categories with $\widetilde{H}: \mathcal{D} \rightarrow$ $\operatorname{Mod}_{\Theta}(\mathcal{C})$. Furthermore, we recall that we have the $\operatorname{map} \operatorname{Mod}_{B}\left(\operatorname{Sp}(G)_{k}\right) \rightarrow$ $\operatorname{Comod}_{(U B, \Gamma)}\left(\operatorname{Sp}_{k}\right)$, which is an equivalence under the assumptions of Theorem 6.11. We can identify the opposite of this map with $\widetilde{U}: \mathcal{C}(G) \rightarrow \operatorname{Mod}_{\Gamma}(\mathcal{C})$. Since $\widetilde{H}$ corresponds to $\widetilde{U} H^{\prime}$ under the equivalence $\operatorname{Fun}\left(\mathcal{D}, \operatorname{Mod}_{\Theta}(\mathcal{C})\right) \stackrel{\simeq}{\rightarrow} \operatorname{Fun}\left(\mathcal{D}, \operatorname{Mod}_{\Gamma}(\mathcal{C})\right)$, we obtain the following corollary. 
Corollary 6.17 Let $G$ be a profinite group that has finite virtual cohomological dimension. We assume that the localization functor $L_{k}$ satisfies Assumption 6.5. If $\Psi_{U B}$ is a $k$-local equivalence, then there is an equivalence of functors

$$
\mathcal{E} x \simeq \operatorname{Coex}
$$

under the equivalence $\operatorname{Mod}_{B}\left(\operatorname{Sp}(G)_{k}\right) \simeq \operatorname{Comod}_{(U B, \Theta)}\left(\operatorname{Sp}_{k}\right)$.

This corollary shows that the formulation of embeddings of module categories in terms of model categories in Proposition 4.5 and that in terms of quasi-categories in Proposition 5.2 are equivalent.

\section{Embeddings over profinite Galois extensions}

We assume that $G$ is a profinite group which has finite virtual cohomological dimension. Furthermore, we assume that the localization functor $L_{k}$ satisfies Assumption 6.5. In this section we show that the two formulations of embeddings of module categories are equivalent if $\varphi: A \rightarrow B$ is a $k$-local $G$-Galois extension.

First, we show that a $k$-local $G$-Galois extension gives an embedding of module categories. Let $\varphi: A \rightarrow B$ be a $k$-local $G$-Galois extension. We have a symmetric monoidal $\Sigma$ Sp-Quillen adjunction

$$
\operatorname{Ex}: \operatorname{Mod}_{A}\left(\Sigma \operatorname{Sp}_{k}\right) \rightleftarrows \operatorname{Mod}_{B}\left(\Sigma \operatorname{Sp}(G)_{k}\right): \operatorname{Re}
$$

by Lemma 4.4, which induces an adjunction of symmetric monoidal Ho( $\Sigma$ Sp)algebras

$$
\mathbb{L E x}: \operatorname{Ho}\left(\operatorname{Mod}_{A}\left(\Sigma \operatorname{Sp}_{k}\right)\right) \rightleftarrows \operatorname{Ho}\left(\operatorname{Mod}_{B}\left(\Sigma \operatorname{Sp}(G)_{k}\right)\right): \mathbb{R R e} .
$$

Let $\mathbf{T}$ be the full subcategory of $\operatorname{Ho}\left(\operatorname{Mod}_{A}\left(\Sigma \operatorname{Sp}_{k}\right)\right.$ consisting of $X$ such that the unit map $X \rightarrow \mathbb{R} \operatorname{Re} L \operatorname{Ex}(X)$ is an isomorphism

$$
\mathbf{T}=\left\{X \in \operatorname{Ho}\left(\operatorname{Mod}_{A}\left(\Sigma \operatorname{Sp}_{k}\right)\right) \mid X \stackrel{\cong}{\longrightarrow} \operatorname{ReLEx}(X)\right\}
$$

Proposition 7.1 If $\varphi: A \rightarrow B$ is a $k$-local $G$-Galois extension, then the restriction of the functor

$$
\mathbb{L E x}: \operatorname{Ho}\left(\operatorname{Mod}_{A}\left(\Sigma \operatorname{Sp}_{k}\right)\right) \longrightarrow \operatorname{Ho}\left(\operatorname{Mod}_{B}\left(\Sigma \operatorname{Sp}(G)_{k}\right)\right)
$$

to the full subcategory $\mathbf{T}$ is fully faithful as an $\mathrm{Ho}(\Sigma \mathrm{Sp})$-enriched functor. Furthermore, if $\varphi$ is a consistent $k$-local $G$-Galois extension, then the full subcategory $\mathbf{T}$ contains all dualizable objects.

Proof The first part follows from Propositions 4.5. If $B$ is a consistent $k$-local $G$ Galois extension of $A$, then $A \rightarrow B^{h G}$ is an equivalence by [4, Prop. 6.1.7(3) and 6.3.1]. Hence the second part follows from Proposition 4.6. 
In the following of this section we shall show that the underlying quasi-category of the model category $\operatorname{Mod}_{B}\left(\Sigma \operatorname{Sp}(G)_{k}\right)$ is equivalent to $\operatorname{Comod}_{(U B, \Theta)}\left(\operatorname{Sp}_{k}\right)$. Now we recall the construction of the map

$$
\Psi_{M}: B(U B, A, M) \rightarrow U \operatorname{Map}_{c}(G, M)
$$

for $M \in \operatorname{Mod}_{U B}(\Sigma \mathrm{Sp})$. The map $\Psi_{M}$ is obtained by applying $U$ to the map $B(B, A, M) \rightarrow \operatorname{Map}_{c}(G, M)$ in $\Sigma \operatorname{Sp}(G)$ that is adjoint to the map $U B(B, A, M) \cong$ $B(U B, A, M) \rightarrow M$ induced by the action of $U B$ on $M$.

Lemma 7.2 If $\varphi: A \rightarrow B$ is a $k$-local G-Galois extension, then the map $\Psi_{M}$ : $B(U B, A, M) \rightarrow U \operatorname{Map}_{c}(G, M)$ is a $k$-local equivalence for any cofibrant and fibrant object $M$ in $\operatorname{Mod}_{U B}\left(\Sigma \operatorname{Sp}_{k}\right)$.

Proof By the definition of $k$-local $G$-Galois extensions [4, Def. 6.2.1], we have a fundamental neighborhood system $\left\{U_{\alpha}\right\}$ of the identity element of $G$ consisting of open normal subgroups and a directed system of finite $k$-local $G_{\alpha}$-Galois extensions $B_{\alpha}$ of $A$, where $G_{\alpha}=G / U_{\alpha}$. By the definition of finite Galois extensions [4, Def. 1.0.1], we have a $k$-local equivalence

$$
B_{\alpha} \wedge_{A} B_{\alpha} \stackrel{\simeq_{k}}{\longrightarrow} \operatorname{Map}\left(G_{\alpha}, B_{\alpha}\right) .
$$

Furthermore, we have an isomorphism $B\left(B_{\alpha}, A, B_{\alpha}\right) \cong B\left(B_{\alpha}, A, A\right) \wedge_{A} B_{\alpha}$ and an equivalence $B\left(B_{\alpha}, A, A\right) \stackrel{\simeq}{\rightarrow} B_{\alpha}$. Since $A \rightarrow B_{\alpha}$ is a cofibration in the category of commutative symmetric ring spectra, we obtain an equivalence $B\left(B_{\alpha}, A, B_{\alpha}\right) \stackrel{\widetilde{\leftrightharpoons}}{\rightarrow}$ $B_{\alpha} \wedge_{A} B_{\alpha}$ by [31, Prop. 15.12]. Hence we obtain a $k$-local equivalence

$$
B\left(B_{\alpha}, A, B_{\alpha}\right) \stackrel{\simeq_{k}}{\longrightarrow} \operatorname{Map}\left(G_{\alpha}, B_{\alpha}\right)
$$

Let $r_{\alpha}: Q_{\alpha} M \rightarrow M$ be a cofibrant replacement in $\operatorname{Mod}_{B_{\alpha}}\left(\Sigma \operatorname{Sp}_{k}\right)$ such that $r_{\alpha}$ is a trivial fibration. We obtain a $k$-local equivalence

$$
B\left(B_{\alpha}, A, Q_{\alpha} M\right) \stackrel{\simeq_{k}}{\longrightarrow} \operatorname{Map}\left(G_{\alpha}, Q_{\alpha} M\right)
$$

as in Lemma 6.13. Since $A$ and $B_{\alpha}$ are cofibrant commutative symmetric ring spectra, we see that $r_{\alpha}$ induces a $k$-local equivalence between $B\left(B_{\alpha}, A, Q_{\alpha} M\right)$ and $B\left(B_{\alpha}, A, M\right)$ by using [31, Prop. 15.12]. Since $r_{\alpha}$ is a trivial fibration, $\operatorname{Map}\left(G_{\alpha}, Q_{\alpha} M\right) \rightarrow \operatorname{Map}\left(G_{\alpha}, M\right)$ is also a trivial fibration. Hence

$$
B\left(B_{\alpha}, A, M\right) \longrightarrow \operatorname{Map}\left(G_{\alpha}, M\right)
$$

is a $k$-local equivalence. Since $\Psi_{M}$ is the colimit of the above maps over the directed system, the lemma follows from Proposition 3.11. 
Theorem 7.3 If $\varphi: A \rightarrow B$ is a $k$-local $G$-Galois extension, then there is an equivalence of quasi-categories

$$
\operatorname{Mod}_{B}\left(\operatorname{Sp}(G)_{k}\right) \simeq \operatorname{Comod}_{(U B, \Theta)}\left(\operatorname{Sp}_{k}\right)
$$

Under this equivalence, there is an equivalence of functors

$$
\mathcal{E} x \simeq \text { Coex }
$$

Proof By Theorem 6.11, we have an equivalence between $\operatorname{Mod}_{B}\left(\operatorname{Sp}(G)_{k}\right)$ and $\operatorname{Comod}_{(U B, \Gamma)}\left(\operatorname{Sp}_{k}\right)$. We can show that $\operatorname{Comod}_{(U B, \Gamma)}\left(\operatorname{Sp}_{k}\right)$ is equivalent to $\operatorname{Comod}_{(U B, \Theta)}\left(\operatorname{Sp}_{k}\right)$ as in Theorem 6.15 by using Lemma 7.2. This completes the proof.

Theorem 7.3 shows that the two formulations of embeddings of module categories are equivalent if $\varphi: A \rightarrow B$ is a $k$-local $G$-Galois extension.

\section{References}

1. Adámek, J., Rosický, J.: Locally Presentable and Accessible Categories. London Mathematical Society Lecture Note Series, vol. 189. Cambridge University Press, Cambridge (1994)

2. Amitsur, S.A.: Simple algebras and cohomology groups of arbitrary fields. Trans. Am. Math. Soc. 90, 73-112 (1959)

3. Boardman, J.M., Vogt, R.V.: Homotopy Invariant Algebraic Structures on Topological Spaces. Lecture Notes in Mathematics, vol. 347. Springer, Berlin (1973)

4. Behrens, M., Davis, D.G.: The homotopy fixed point spectra of profinite Galois extensions. Trans. Am. Math. Soc. 362(9), 4983-5042 (2010)

5. Bousfield, A.K.: The localization of spectra with respect to homology. Topology 18(4), 257-281 (1979)

6. Bousfield, A.K.: On the homotopy theory of K-local spectra at an odd prime. Am. J. Math. 107(4), 895-932 (1985)

7. Carlsson, G.: Derived completions in stable homotopy theory. J. Pure Appl. Algebra 212(3), 550-577 (2008)

8. Ching, M., Riehl, E.: Coalgebraic models for combinatorial model categories. Homol. Homot. Appl. 16(2), 171-184 (2014)

9. Davis, D.G.: Homotopy fixed points for $L_{K}(n)\left(E_{n} \wedge X\right)$ using the continuous action. J. Pure Appl. Algebra 206(3), 322-354 (2006)

10. Davis, D.G., Torii, T.: Every $K(n)$-local spectrum is the homotopy fixed points of its Morava module. Proc. Am. Math. Soc. 140(3), 1097-1103 (2012)

11. Devinatz, E.S., Hopkins, M.J.: Homotopy fixed point spectra for closed subgroups of the Morava stabilizer groups. Topology 43(1), 1-47 (2004)

12. Devinatz, E.S., Hopkins, M.J., Smith, J.H.: Nilpotence and stable homotopy theory. I. Ann. Math. (2) 128(2), 207-241 (1988)

13. Dugger, D.: Combinatorial model categories have presentations. Adv. Math. 164(1), 177-201 (2001)

14. Franke, J.: Uniqueness theorems for certain triangulated categories possessing an Adams spectral sequence. Preprint (1996). Available at http://www.math.uiuc.edu/K-theory/0139/. Accessed 23 Dec 2016

15. Goerss, P.G.: Homotopy fixed points for Galois groups. The Čech centennial (Boston, MA, 1993), 187-224, Contemp. Math., 181, Am. Math. Soc., Providence, RI (1995)

16. Goerss, P.G., Jardine, J.F.: Simplicial Homotopy Theory. Progress in Mathematics, vol. 174. Birkhäuser Verlag, Basel (1999)

17. Hess, K.: A general framework for homotopic descent and codescent. Preprint 2010. arXiv:1001.1556

18. Hirschhorn, P.S.: Model Categories and Their Localizations. Mathematical Surveys and Monographs, vol. 99. American Mathematical Society, Providence, RI (2003) 
19. Hopkins, M.J., Smith, J.H.: Nilpotence and stable homotopy theory. II. Ann. Math. (2) 148(1), 1-49 (1998)

20. Hovey, M.: Model Categories. Mathematical Surveys and Monographs, vol. 63. American Mathematical Society, Providence, RI (1999)

21. Hovey, M.: Spectra and symmetric spectra in general model categories. J. Pure Appl. Algebra 165(1), 63-127 (2001)

22. Hovey, M., Sadofsky, H.: Invertible spectra in the $E(n)$-local stable homotopy category. J. Lond. Math. Soc. (2) 60(1), 284-302 (1999)

23. Hovey, M., Strickland, N.P.: Morava K-theories and localisation. Mem. Am. Math. Soc. 139, 666 (1999)

24. Hovey, M., Shipley, B., Smith, J.: Symmetric spectra. J. Am. Math. Soc. 13(1), 149-208 (2000)

25. Joyal, A.: Quasi-categories and Kan complexes. J. Pure Appl. Algebra 175(1-3), 207-222 (2002)

26. Joyal, A.: The Theory of Quasi-categories and its Applications, vol. II. pp. 153-496 (2008)

27. Lurie, J.: Higher Topos Theory. Annals of Mathematics Studies, vol. 170. Princeton University Press, Princeton, NJ (2009)

28. Lurie, J.: Higher algebra (version May 16, 2016). Available at http://www.math.harvard.edu/ lurie/. Accessed 23 Dec 2016

29. Miller, H.R., Ravenel, D.C., Wilson, W.S.: Periodic phenomena in the Adams-Novikov spectral sequence. Ann. Math. (2) 106(3), 469-516 (1977)

30. Morava, J.: Noetherian localisations of categories of cobordism comodules. Ann. Math. (2) 121(1), 1-39 (1985)

31. Mandell, M.A., May, J.P., Schwede, S., Shipley, B.: Model categories of diagram spectra. Proc. Lond. Math. Soc. (3) 82(2), 441-512 (2001)

32. Raptis, G., Rosický, J.: The accessibility rank of weak equivalences. Theory Appl. Categ. 30(19), 687-703 (2015)

33. Ravenel, D.C.: Nilpotence and Periodicity in Stable Homotopy Theory. Annals of Mathematics Studies, vol. 128. Princeton University Press, Princeton, NJ (1992)

34. Rognes, J.: Galois extensions of structured ring spectra. Mem. Am. Math. Soc. 192(898), 1-97 (2008)

35. Schwede, S., Shipley, B.: Algebras and modules in monoidal model categories. Proc. Lond. Math. Soc. (3) 80(2), 491-511 (2000)

36. Schwede, S., Shipley, B.: Stable model categories are categories of modules. Topology 42(1), 103-153 (2003)

37. Shipley, B.: Symmetric spectra and topological Hochschild homology. $K$-Theory 19(2), 155-183 (2000)

38. Shipley, B.: A convenient model category for commutative ring spectra. Homotopy theory: relations with algebraic geometry, group cohomology, and algebraic $K$-theory, Contemp. Math., vol. 346, pp. 473-483, Am. Math. Soc., Providence, RI (2004)

39. Torii, T.: Quasi-categories of comodules and Landweber exactness, preprint. arXiv:1612.03265 (2016)

40. Wolff, H.: $\mathbb{V}$-localizations and $\mathbb{V}$-monads. J. Algebra 24, 405-438 (1973) 